\title{
Polarized Raman Scattering Studies of Orientational Order in Uniaxial Liquid Crystalline Phases
}

\section{Citation}

Jen, Shen, Noel A. Clark, Peter S. Pershan, and E. B. Priestley. 1977. Polarized Raman scattering studies of orientational order in uniaxial liquid crystalline phases. Journal of Chemical Physics 66(10): 4635-4661.

\section{Published Version}

doi:10.1063/1.433720

\section{Permanent link}

http://nrs.harvard.edu/urn-3:HUL.InstRepos:10361969

\section{Terms of Use}

This article was downloaded from Harvard University's DASH repository, and is made available under the terms and conditions applicable to Other Posted Material, as set forth at http:// nrs.harvard.edu/urn-3:HUL.InstRepos:dash.current.terms-of-use\#LAA

\section{Share Your Story}

The Harvard community has made this article openly available.

Please share how this access benefits you. Submit a story.

\section{Accessibility}




\title{
Polarized Raman scattering studies of orientational order in uniaxial liquid crystalline phases*
}

\author{
Shen Jen, ${ }^{\dagger}$ Noel A. Clark, and P. S. Pershan \\ Division of Engineering and Applied Physics, Harvard University. Cambridge, Massachusetts 02138 \\ E. B. Priestley \\ RCA Laboratories, Princeton, New Jersey 08540
}

(Received 14 September 1976)

\begin{abstract}
The measurement of vibrational Raman depolarization ratios has been used to study molecular orientational order in uniaxial single domain nematic and smectic liquid crystal samples. This technique is demonstrated to obtain the same microscopic order parameter $\left\langle P_{2}\right\rangle=1 / 2\left\langle 3 \cos ^{2} \theta-1\right\rangle$, where $\theta$ is the angle between a molecular long axis and the uniaxial direction, as other existing methods. In addition, the next higher moment of the orientational distribution function $\left\langle P_{4}\right\rangle=1 / 8\left\langle 35 \cos ^{4} \theta-30 \cos ^{2} \theta+3\right\rangle$ has been measured for the first time. The physical basis, theoretical apparatus, and experimental methods necessary for the application of this technique are thoroughly detailed in this paper. Measurements are presented of the temperature dependence of $\left\langle P_{2}\right\rangle$ and $\left\langle P_{4}\right\rangle$ of $N$-( $p^{\prime}$-butoxybenzylidene)-pcyanoaniline (BBCA) dissolved in $N$ - $\left(p^{\prime}\right.$-methoxybenzylidene)-p-cyanoaniline (MBBA) and of pure MBBA in the isotropic and nematic phases, and in the isotropic, nematic, smectic $A$, and smectic $B$ phases of $N-\left(p^{\prime}\right.$-butoxybenzylidene)- $p-n$-octylaniline (40.8). In the nematic phases the new quantitative information marks significant discrepancies with existing theories of nematic ordering. In the smectic phases the results show unambiguously the anticipated high degree of molecular orientational order.
\end{abstract}

\section{INTRODUCTION}

In the earliest attempts to explain the anisotropic properties of nematic liquid crystals, the molecules were assumed to be rigid rods with cylindrical symmetry. It was also assumed that the symmetry axis of any individual molecule made an angle $\theta$ with the macroscopic symmetry axis of the nematic phase and theoretical efforts were directed towards prediction of an anisotropic angular distribution function $f(\cos \theta)$. In the case of an isotropic phase, all molecular orientations are equally probable and $f(\cos \theta)$ is constant. A principal result of any of these calculations is a theoretical prediction for the statistical average of the Legendre polynomials $P_{2 l}(\cos \theta)$,

$$
\left\langle P_{2 l}(\cos \theta)\right\rangle=\int_{0}^{\pi} \sin \theta d \theta P_{2 l}(\cos \theta) f(\cos \theta)
$$

which, being nonzero only if the system is anisotropic, may be viewed as orientational order parameters. ${ }^{1,2}$ Of these $\left\langle P_{2}\right\rangle$ has been of particular interest since, until recently, only $\left\langle P_{2}\right\rangle$ has been accessible experimentally. The purpose of this paper is to fully describe a technique reported briefly in a letter ${ }^{(3)}$ by which $\left\langle P_{2}\right\rangle$ and $\left\langle P_{4}\right\rangle$ can be simultaneously determined.

Molecular field theories such as that of Maier and Saupe predict a temperature dependence of $\left\langle P_{2}\right\rangle$ which is in reasonably good qualitative agreement with numerous experimental results. ${ }^{1}$ Residual quantitative discrepancies have prompted attempts to improve on the MaierSaupe calculation in a variety of ways. One approach is to add more terms and thereby more adjustable parameters to the mean field. ${ }^{2}$ In this way calculated values of $\left\langle P_{2}\right\rangle$ in essentially perfect quantitative agreement with experiment can be obtained. We will show, however, that the associated theoretical values of $\left\langle P_{4}\right\rangle$ differ substantially from our experimental results.
A second question raised in a number of recent theoretical papers ${ }^{4}$ concerns the assumption that liquid crystal molecules can be treated as axially symmetric rigid rods. In place of the simple angular distribution function $f(\cos \theta)$, these papers propose a more general expression in terms of the Eulerian angles necessary to specify the orientation of molecules of lower symmetry. In principle, this criticism of the earlier work is correct and there is experimental evidence supporting the assertion that corrections due to local biaxiality are of some practical importance. ${ }^{5}$ On the other hand, there is also experimental evidence to the effect that liquid crystal molecules execute rotational motions about their long axis, ${ }^{6}$ suggesting weak local biaxiality. In any event, we have considered the effects of local biaxiality on our Raman measurements and conclude that, even if such effects were present, they would not significantly alter the measured values of $\left\langle P_{2}\right\rangle$ and $\left\langle P_{4}\right\rangle$. To put this another way, the present measurements are relatively insensitive to local biaxiality.

Another problem with the mean field calculations is that liquid crystal molecules are not rigid rods. Typical molecules have a rigid central section with somewhat flexibile hydrocarbon "tails" attached to either one or both ends of the rigid section. This is not very serious for the experiments since they presumably measure the angular distribution of the rigid central part. It could, however, be a source of discrepancy between experiment and those theoretical calculations that assume rigid molecules.

Finally, the Maier-Saupe calculation ignores shortrange molecular interactions which determine the nature of orientational pair correlations. The influence of short-range order on $\left\langle P_{2 l}(\cos \theta)\right\rangle$ has been discussed by Sheng and Wojtowicz. ${ }^{7}$

A satisfactory determination of the importance of 
(a)

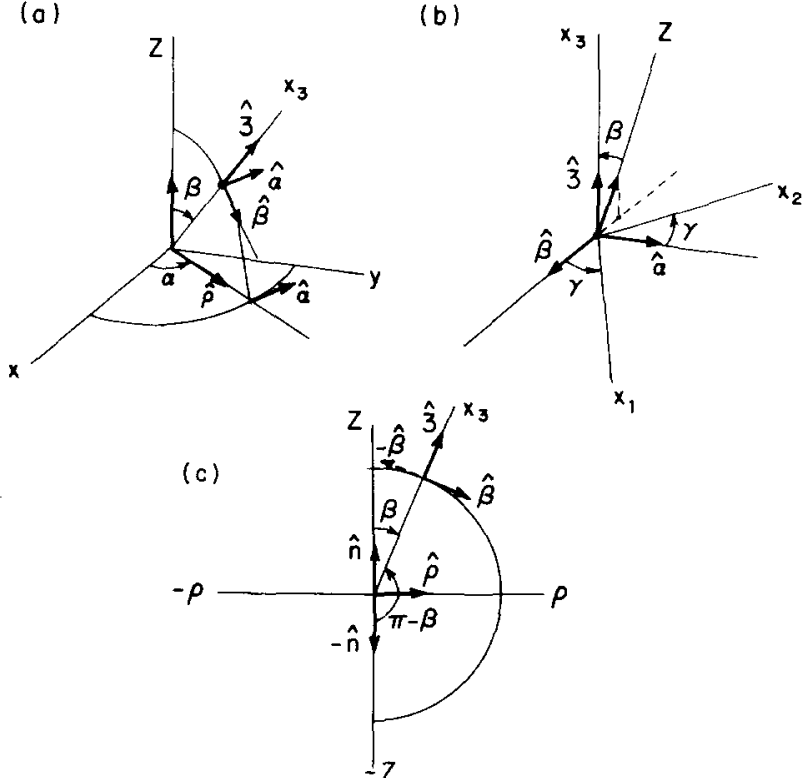

FIG. 1. (a) and (b) show the orientation between the laboratory and the molecular frames, that is $\Omega=(\alpha, \beta, \gamma)$. (c) illustrates the symmetry relation $f(\alpha, \beta, \gamma)=f(\pi+\alpha, \pi-\beta, \pi+\gamma)$ under $\hat{n} \rightarrow-\hat{n}$. The origins are shifted for convenience.

these various effects on the properties of the nematic phase will require experimental determination of the orientational distribution function of each molecular part. This in turn requires the determination of a large number of moments (e.g. , $\left.\left\langle P_{2 l}(\cos \theta)\right\rangle\right)$ of several orientational distribution functions, a ponderous experimental task. This work represents a small step in this direction.

\section{BACKGROUND}

\section{A. Orientational statistics-microscopic order parameters}

We begin with the assumption that the molecules are rigid but of arbitrary shape. As mentioned above, this may not be strictly true but it is probably satisfactory for the central portion of the molecules. Consider two coordinate systems: (1) the laboratory frame $(x, y, z)$ fixed with respect to the liquid crystal and chosen to reflect the macroscopic symmetry of the system, the $z$ axis being parallel to the average director $\hat{n}$; and (2) the molecular frame $(1,2,3)$ fixed on the molecule and chosen by considering the molecular symmetry. The 3 -axis is parallel to the major molecular axis (usually along the longest dimension).

At any instant, due to thermal agitation, the orientation of a particular molecule is described by the three Euler's angles $(\alpha, \beta, \gamma)$ that link the two coordinate systems, as illustrated in Figs. 1(a) and 1(b). The orientational order of the molecules is then described by a distribution function which can be expanded in terms of the generalized spherical harmonics, the Wigner ma$\operatorname{trices}^{\beta} D_{m^{\prime} m}^{(L)}(\alpha, \beta, \gamma)$ :

$f(\alpha, \beta, \gamma)=\sum_{L=0}^{\infty} \sum_{m=-L}^{L} \frac{2 L+1}{8 \pi^{2}} a_{m^{\prime} m}^{(L)} D_{m^{\prime} m}^{(L)}(\alpha, \beta, \gamma)$,

with

$$
a_{m=m}^{(L)}=\int_{0}^{2 \pi} d \alpha \int_{0}^{\pi} \sin \beta d \beta \int_{0}^{2 \pi} d \gamma D_{m^{\prime} m}^{(L) *}(\alpha, \beta, \gamma) f(\alpha, \beta, \gamma)
$$

$$
=\left\langle D_{m^{\prime} m}^{(L)^{*}}(\alpha, \beta, \gamma)\right\rangle
$$

where $\langle\cdots\rangle$ represents a thermal average. It follows that $a_{00}^{(0)}=1$ and the values of the remaining coefficients $a_{m^{\prime} m}^{(L)}$ with nonzero $L$ depend on the degree of orientational ordering. They are all identically zero in the isotropic phase, where $f=1 / 8 \pi^{2}$ over all orientations, and some will be nonzero in the ordered phases. They vary with temperatures and saturate to constant values if the system becomes completely aligned. It is therefore natural to choose the $a_{m^{\prime} m}^{(L)}$ as a set of generalized orientational order parameters that characterize the amount of ordering. By observing the symmetries of the $D_{m^{\prime} m}^{(L)}$ and the fact that $f(\alpha, \beta, \gamma)$ is real, one can show that the $a_{m^{\prime} m}^{(L)}$ are complex conjugate pairs and obey a symmetry relation similar to that of the $D^{\prime} \mathrm{s}$. Thus, in general, for each $L$, there are $(2 L+1)^{2}$ independent real orientational order parameters. In fact, this set of $(2 L+1)^{2}$ parameters for a particular $L$ is all that is required to relate the average of any $L$ th rank irreducible tensor to its molecular value.

The distribution function, Eq. (1), can be greatly simplified and the number of nontrivial order parameters considerably reduced by imposing the known symmetries of the macroscopic liquid crystal phase and of the individual constituent molecules. For a uniaxial macroscopic phase with the unique axis parallel to the $z$ axis, the distribution function is independent of $\alpha$, and only terms with $m^{\prime}=0$ contribute to the summation. Furthermore, evidenced by the lack of ferroelectricity in the mesophase of interest, $\hat{n}$ and $-\hat{n}$ are equivalent physical states. This implies [Fig. 1(c)] that $f(\alpha, \beta, \gamma)$ $=f(\pi+\alpha, \pi-\beta, \pi+\gamma)$, whence the coefficients of all the odd $L$ terms in Eq. (1) are zero. We have then

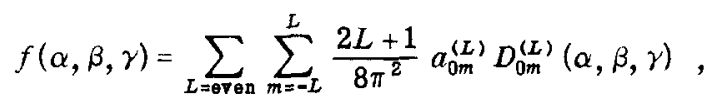

where

$$
D_{0 m}^{(L)}(\alpha, \beta, \gamma)=\left(\frac{4 \pi}{2 L+1}\right)^{1 / 2}(-1)^{m} Y_{L m}^{*}(\beta, \gamma) .
$$

Therefore, for rigid molecules of arbitrary shape forming a uniaxial liquid crystal we have $(2 L+1)$ real, independent order parameters for each $L$, and $L$ takes on only even integral values. To lowest order in $L$, that is $L=2$, there are five nontrivial parameters that are commonly acknowledged in various theoretical and experimental works. We point out that a more familiar definition, introduced by Saupe, ${ }^{9}$

$$
S_{i j}^{\alpha \beta}=\frac{1}{2}\left\langle 3 i_{\alpha} j_{\beta}-\delta_{i j} \delta_{\alpha \beta}\right\rangle \text {, }
$$

with $\alpha, \beta=x, y, z, i, j=1,2,3$, and $i_{\alpha}=\hat{i} \cdot \hat{\alpha}$, is equivalent to the set of generalized order parameters of $L=2$ given by Eq. (1b). Repeated indices are summed over throughout the paper. Under the same symmetry arguments mentioned above the independent components of Eq. (3), $S_{i j}^{\alpha x}$, form a second rank irreducible tensor whose components, when expressed in the spherical tensor notation, are given by the five coefficients with $L=2$ in Eq. (2). (See, for example, Ref. 10.) This tensor is called the microscopic tensor order parameter $\mathbf{S}$.

Writing Eq. (2) explicitly, we have 


$$
\begin{aligned}
f(\alpha, \beta, \gamma)= & \left(1 / 8 \pi^{2}\right)\left[1+\frac{5}{2} A_{0}^{(2)}\left(3 \cos ^{2} \beta-1\right)+5 \sqrt{6} \sin \beta \cos \beta\left(A_{1}^{(2)} \cos \gamma+B_{1}^{(2)} \sin \gamma\right)+5 \sqrt{\frac{3}{2}} \sin ^{2} \beta\left(A_{2}^{(2)} \cos 2 \gamma+B_{2}^{(2)} \sin 2 \gamma\right)\right. \\
& +\frac{9}{8} A_{0}^{(4)}\left(35 \cos ^{4} \beta-30 \cos ^{2} \beta+3\right)+\frac{9}{2} \sqrt{5} \sin \beta \cos \beta\left(7 \cos ^{2} \beta-3\right)\left(A_{1}^{(4)} \cos \gamma+B_{1}^{(4)} \sin \gamma\right)+\frac{9}{2} \sqrt{\frac{5}{2}} \sin ^{2} \beta\left(7 \cos ^{2} \beta-1\right) \\
& \left.\times\left(A_{2}^{(4)} \cos 2 \gamma+B_{2}^{(4)} \sin 2 \gamma\right)+\frac{9}{2} \sqrt{35} \sin ^{3} \beta \cos \beta\left(A_{3}^{(4)} \cos 3 \gamma+B_{3}^{(4)} \sin 3 \gamma\right)+\frac{9}{4} \sqrt{\frac{35}{2}} \sin ^{4} \beta\left(A_{4}^{(4)} \cos 4 \gamma+B_{4}^{(4)} \sin 4 \gamma\right)+\cdots\right]
\end{aligned}
$$

where the complex $a_{0 m}^{(L)}$ and $D_{0 m}^{(L)}(\alpha, \beta, \gamma)$ have been combined into real quantities by defining

$$
a_{0 m}^{(L)} \equiv(-1)^{m} a_{0,-m}^{(L) *}=A_{m}^{(L)}+i B_{m}^{(L)} .
$$

The explicit form of the real order parameters for $L=2$ and 4 is given in Appendix II. For molecules having no plane of symmetry, all the $(2 L+1)$ parameters for each $L$ are expected to be nonzero, with the $A_{0}^{(L)}$ characterizing the "axial" ordering with respect to the director and the others the "azimuthal" ordering around the molecu- lar axis. If the molecule has a single plane of symmetry, and the 1 axis is perpendicular to this plane, $f(\alpha, \beta, \gamma)=f(\alpha, \beta, \pi-\gamma)$. This reduces the number of nontrivial terms in Eq. $(2 \mathrm{a})$ to $(L+1)$ for each $L$. The number of nonzero order parameters is similarly reduced. If the molecule has two mutually perpendicular planes of symmetry, then choosing the 3-axis to be parallel to both obtains a further symmetry relation $f(\alpha, \beta, \gamma)=f(\alpha, \beta, \pi+\gamma)$. This eliminates $L / 2$ terms and there remain only $(1+L / 2)$ nontrivial terms in the summation.

$$
\begin{gathered}
f(\alpha, \beta, \gamma)=\left(1 / 8 \pi^{2}\right)\left[1+\frac{5}{2} A_{0}^{(2)}\left(3 \cos ^{2} \beta-1\right)+5 \sqrt{\frac{3}{2}} A_{2}^{(2)} \sin ^{2} \beta \cos 2 \gamma+\frac{9}{8} A_{0}^{(4)}\left(35 \cos ^{4} \beta-30 \cos ^{2} \beta+3\right)\right. \\
\left.+\frac{9}{2} \sqrt{\frac{5}{2}} A_{2}^{(4)} \sin ^{2} \beta\left(7 \cos ^{2} \beta-1\right) \cos 2 \gamma+\frac{9}{4} \sqrt{\frac{35}{2}} A_{4}^{(4)} \sin ^{4} \beta \cos 4 \gamma+\cdots\right]
\end{gathered}
$$

If the molecule has a threefold or greater axis of symmetry, then taking this axis as the 3 -axis results in only one nontrivial term for each $L$, that is, $Y_{L O}(\beta, \gamma)$ $=[(2 L+1) / 4 \pi]^{1 / 2} P_{L}(\cos \beta)$. The simple assumption that has been commonly adopted is that the liquid crystals are made up by cylindrically symmetrical rigid rods. This obtains

$$
f(\alpha, \beta, \gamma)=\sum_{L=\text { ơ en }} \frac{2 L+1}{8 \pi^{2}} A_{0}^{(L)} P_{L}(\cos \beta)
$$

or

$$
\begin{aligned}
f_{\hat{u}}(\beta) & =\int_{0}^{2 \mathrm{r}} d \alpha \int_{0}^{2 \pi} d \gamma f(\alpha, \beta, \gamma) \\
& =\sum_{L=\text { ovon }} \frac{2 L+1}{2} A_{0}^{(L)} P_{L}(\cos \beta)
\end{aligned}
$$

and

$$
A_{0}^{(L)}=\int_{0}^{\pi} \sin \beta d \beta P_{L}(\cos \beta) f_{\hat{u}}(\beta)=\left\langle P_{L}(\cos \beta)\right\rangle,
$$

where $\hat{u}$ is the unit vector along the symmetry axis of the molecule. The order parameters $A_{0}^{(L)}$ vanish in the isotropic phase, take nonzero values in the ordered phase, and saturate to unity when the system becomes completely aligned.

\section{B. Macroscopic order parameters}

Microscopic order parameters of the type just described are usually the primary results of model calculations. On the other hand, many measurements relate most directly to macroscopic quantities and it is necessary to inquire about the relationship between the two.

Due to the long-range orientational order of the liquid crystalline mesophases, most macroscopic tensor properties show some anisotropy. For example, any of the phenomenological second rank tensors could be chosen to describe the degree of ordering. We take a general second rank tensor $\mathbf{T}$ and define a macroscopic tensor order parameter $\mathbf{Q}$ as the anisotropic part of $\mathbf{T}^{11}$ :

$$
\mathbf{Q}=C \mathbf{T}^{a}=C\left(\mathbf{T}-\frac{1}{3} \mid \operatorname{tr} \mathbf{T}\right),
$$

where the constant $C$ can be chosen according to the convention that $Q_{z z}$ saturates to unity in a completely aligned state. This definition is quite general and applies to both uniaxial and biaxial systems. In a uniaxial system there is only one independent component. Note that, although any phenomenological $\mathbf{T}$ could be used as long as it shows a characteristic dependence upon the orientational ordering, it is not obvious that they will yield equivalent values for $Q$. As a matter of fact, we want an order parameter $\mathbf{Q}$ that not only serves to characterize the degree of macroscopic order but one that can also be related to its microscopic counterpart. For reasons that will be discussed shortly, the magnetic susceptibility $\chi$ seems to be the most suitable macroscopic quantity to substitute for $\mathbf{T}$ in $\mathbf{E q}$. (8).

Provided the molecules can be treated as approximately rigid, there exists a connection between $Q$ and the $a_{m^{\prime} m}^{(L)}$ defined above. The subtle point here involves a relation between macroscopic and microscopic (molecular) tensor properties, known as the local field correction for either electric or magnetic fields. The local field correction for the magnetic response is negligible because interactions among molecules are small (the diamagnetic susceptibility of typical mesogenic molecules is of the order of $10^{-7} \mathrm{cgs}$ units). $\chi$ is then simply a sum over all molecules with susceptibility $\zeta$ per molecule,

$$
\chi=\sum_{\text {moleculos }} \zeta=N \quad d \Omega f(\Omega) \zeta=N\langle\zeta\rangle,
$$


where $N$ is the total number of molecules and $\boldsymbol{\chi}$ magnetic susceptibility per unit mass. Using the well known transformation properties of irreducible tensor operators $\hat{\boldsymbol{\beta}}_{m}^{(L)}$, one can write ${ }^{12}$

$$
\mathbf{Q}=C \chi^{a}=C N \hat{\boldsymbol{\beta}}_{0}^{(2)} \sum_{m=-2}^{2} \zeta(2, m)\left\langle D_{0 m}^{(2) *}(\alpha, \beta, \gamma)\right\rangle,
$$

where the $\zeta(2, m)$ are the coefficients of the $\hat{\beta}_{m}^{(2)}$ when the molecular susceptibility is expanded in terms of the irreducible tensors in the molecular frame. Alternatively, Eq. (10) can be expressed as

$$
Q_{\alpha \beta}=C\left(\chi_{\alpha \beta}-\frac{1}{3} \delta_{\alpha \beta} \chi_{\gamma \gamma}\right)=C N\left(\hat{\beta}_{0}^{(2)}\right)_{\alpha \beta} \sum_{m=-2}^{z} \zeta(2, m) a_{0 m}^{(2)},
$$

with $C$ given by

$$
C=3 / N\left(2 \zeta_{33}-\zeta_{11}-\zeta_{22}\right)
$$

Equation (11) is the desired relation between the macroscopic order parameter $\mathbf{Q}$ and the microscopic parameters $a_{0 m}^{(2)}$ defined by Eq. (1).

For the electric response it is necessary, though difficult, to evaluate the nontrivial contribution from all other dipoles in the medium. Satisfactory results exist only for cubic crystals and isotropic fluids. For crystals of lower symmetry, theoretical calculations are quite complicated and the results are less informative. ${ }^{13}$ Attempts to evaluate local field corrections for liquid crystals have been even less fruitful. ${ }^{14}$ This is why the macrocopic $Q$ is best defined through $\chi$ as we mentioned earlier. However, empirical local field corrections have frequently been used and it has been pointed out ${ }^{15}$ that the one proposed by Vuks, ${ }^{16}$ and used to interpret data from light scattering and Kerr effect experiments on other systems, presumably also works for liquid crystals. This amounts to a modified Clausius-Mossotti relation in the optical frequency region,

$$
\left(\epsilon_{\beta}-1\right) /(\bar{\epsilon}+2)=\frac{4}{3} \pi N_{0}\left\langle\alpha_{\beta}\right\rangle,
$$

where $\epsilon_{\beta}$ and $\alpha_{\beta}$ are the optical dielectric constant and effective molecular polarizability along the principal $\beta$ axis, $\bar{\epsilon}$ is the average optical dielectric constant, and $N_{0}$ is the number of molecules per unit volume. Equation (13) implies a local field correction in the liquid crystal phase similar to that in the isotropic phase. Local field corrections depend on dipole-dipole summations over neighboring molecules and although they may be calculated in a number of ways, the answers are ultimately dependent on near-neighbor correlations. These near-neighbor correlations appear to be relatively insensitive to temperature, implying the same about the local field corrections. Some indication along this line has recently been observed ${ }^{17}$ in $x$-ray diffraction studies on liquid crystals.

It follows that a semiempirical macroscopic tensor order parameter $Q^{\prime}$, defined in terms of the optical dielectric anisotropy, can be related similarly to the microscopic $a_{m^{\prime} m}^{(L)}$ via $\mathrm{Eq} .(13)$ and the molecular polarizability $\boldsymbol{\alpha}$. We have

$$
\boldsymbol{\epsilon}^{a}=\frac{4}{3} \pi N_{0}(\overline{\boldsymbol{\epsilon}}+2)\left\langle\boldsymbol{\alpha}^{a}\right\rangle,
$$

and

$$
\mathbf{Q}^{\prime}=C^{\prime} \boldsymbol{\epsilon}^{a}=C^{\prime} \frac{4}{3} \pi N_{0}(\overline{\boldsymbol{\epsilon}}+2) \hat{\boldsymbol{\beta}}_{0}^{(2)} \sum_{m=-2}^{2} \alpha(2, m)\left\langle D_{0 m}^{(2) *}(\alpha, \beta, \gamma)\right\rangle,
$$

or

$$
\begin{aligned}
Q_{\alpha \beta}^{\prime}=C^{\prime}\left(\epsilon_{\alpha \beta}-\frac{1}{3} \delta_{\alpha \beta} \epsilon_{\gamma \gamma}\right)= & C^{\prime} \frac{4}{3} \pi N_{0}(\bar{\epsilon}+2)\left(\hat{\beta}_{0}^{(2)}\right)_{\alpha \beta} \\
& \times \sum_{m=-2}^{2} \alpha(2, m) a_{0 m}^{(2)},
\end{aligned}
$$

with

$$
C^{\prime}=9 / 4 \pi N_{0}(\bar{\epsilon}+2)\left(2 \alpha_{33}-\alpha_{11}-\alpha_{22}\right) .
$$

In the absence of empirical evidence it is far from certain that $\mathbf{Q}^{\prime}$ is the same as $\mathbf{Q}$.

\section{Review of experimental studies}

There have been many experiments done in the past to study the long-range orientational order in liquid crystals, particularly in nematic liquid crystals. Most of these experiments have been based on observations of the average properties of second rank tensors and have thus led to determinations of the order parameters $\mathbf{Q}$ and $a_{0 m}^{(2)}$ introduced above. A brief summary of these experiments follows.

(1) Macroscopic order parameter: Measurement of the magnetic susceptibility. The determination of $\mathbf{Q}$ is unambiguous in the sense that there is just one independent component and the result is independent of molecular structure. On the other hand, the need for data in the crystalline phase, in order to determine the absolute magnitude of $\boldsymbol{a}$, is a serious drawback. The anisotropy of magnetic susceptibility has been measured both in nematic ${ }^{18}$ and smectic $A^{19}$ phases.

(2) Microscopic order parameter: Magnetic resonance. Magnetic resonance provides a direct measurement of $\mathbf{Q}$ since the analysis does not require data in the other phases. Splittings due to the dipolar interaction in NMR, and the quadrupolar interaction in NQR, give unambiguous information about the $a_{0 m}^{(2)}$. Interpretation of the experimental data is complicated by the fact that in reality most mesogens have very low molecular symmetry and the measured splittings depend on all five of the $L=2$ order parameters $a_{0 m}^{(2)}$ of Eq. (2). The situation can be simplified by choosing a solute molecule of known symmetry and studying its order parameters (which will number fewer than five, depending on the symmetry of the molecule). The order parameters of the solute molecule give some indication of the degree of ordering of the host provided the solute molecule aligns in the liquid crystal. However, there is no direct relation between the order parameters of the solute and those of the host. ${ }^{20}$ In an analogous way paramagnetic solute molecules have been used in EPR experiments to give similarly qualitative information about the host ordering. ${ }^{21}$ Proton magnetic resonance experiments have been performed on both nematic ${ }^{22}$ and smectic ${ }^{23}$ phases and the value of $A_{0}^{(2)}$ estimated by calculating the second moment of the broadline proton absorption spectrum under the assumption of rapid reorientation around the major molecular axis. There remains one question concerning the flexibility of the mol- 
ecules. This can be answered by NMR of selectively deuterated molecules ${ }^{24}$ and/or by NQR, ${ }^{25}$ in which the splittings originate in specific, identifiable sections of the molecules. Most such work has been concentrated on the relatively rigid central core of the molecules.

(3) Measurements of $\mathbf{Q}^{\prime}$ or $a_{0 m}^{(2)}$ based on the empirical local field correction. With the empirical local field correction and data in the crystalline phase, $A_{0}^{(2)}$ can also be determined from the optical indices of refraction, ${ }^{26}$ that is from the optical dielectric anisotropy, or from ir and uv absorption measurements. ${ }^{27}$ The results from the optical dielectric anisotropy are found to be very close to those obtained by NMR. Presumably the central core region of the molecule with its higher electron density and lower excitation energy makes the dominant contribution to the optical dielectric tensor. Also, qualitative information has been obtained by measuring polarized fluorescence and pleochroic absorption of a probe dye molecule dissolved in the liquid crystal host. ${ }^{28}$

(4) More general studies. A direct method of measuring the full $f_{\hat{u}}(\beta)$ by large angle $\mathrm{x}$-ray or neutron scattering has been proposed in the nematic phase. ${ }^{29}$ With large scattering vectors the interference effect among molecules becomes negligible if the wavelength of the incident photons or neutrons is much shorter than the molecular diameter. Then, if one knows the molecular scattering factor one can, in principle deduce $f_{\hat{u}}(\beta)$ by deconvoluting the observed intensity distribution. Experiments utilizing this principle have not yet been fully exploited. ${ }^{30}$

\section{ORIENTATIONAL ORDER BY RAMAN SCATTERING}

It is well known that Raman scattering can be employed to obtain quantitative information on rotational and other kinetic behavior of molecules in condensed matter as well as gases of high density. The power spectrum of the scattered light can be expressed as a fourth rank tensor which is related to the average orientational correlation function of the molecular Raman polarizability. This suggests that application of Raman scattering to the liquid crystal system will yield not only the order parameters $a_{0 m}^{(2)}$, as in the conventional methods, but also information of next higher order, that is, the terms with $L=4$ in the previous discussion. ${ }^{31}$

The simplest theoretical framework for discussion of the present experiments is Placzek's polarizability theory of Raman scattering. ${ }^{32}$ Consider a single Raman active vibrational mode with normal coordinate $Q$ and eigenfrequency $\omega$. Following Placzek we introduce a Raman polarizability tensor $\alpha^{\prime}$, whose time dependence in a reference frame fixed with respect to the molecule follows that of $Q$,

$$
\alpha^{\prime}=(\partial \alpha / \partial Q)_{Q=0} Q \text {. }
$$

If the components of $\alpha^{\prime}$ are expressed relative to the laboratory reference frame, they will have additional time dependence due to the rotational motions of the molecule. In principle there is a further complication in that we take $\alpha^{\prime}$ to be a macroscopic quantity in the sense that it relates the macroscopic incident electric field to the macroscopic radiation field. If, however, $\alpha^{\prime}$ were interpreted as a microscopic property of the bare molecule, one could reasonably ask whether or not local field corrections were needed in order to connect the macroscopic properties to the microscopic. Quantitative theoretical calculations of local field corrections are exceedingly difficult and if they were needed here, we would be hard pressed to proceed further. A similar local field problem exists for the conventional optical dielectric constants and, as we discussed above, both Vuks $^{16}$ and Chandrasekhar ${ }^{15}$ have pointed out that empirical evidence suggests these are not important. We will present empirical evidence below supporting the contention that local field factors are also not needed in the Raman problem. The explanation, we believe, is that local field corrections ultimately depend on the short range correlations between molecules and that these short range correlations are not significantly different in the isotropic liquid, the nematic liquid crystal, the smectic liquid crystal, and even the crystalline phases. Thus the $\alpha^{\prime}$ in Eq. (18) can be treated as a "dressed" molecular polarizability in the sense that it already includes the local field corrections. This particular point should receive more theoretical attention although we believe it can be justified on the basis of existing work. ${ }^{33}$ In any event, we will present empirical evidence in support of the hypothesis that for each of the molecular vibrations of interest here there is a tensor $\alpha^{\prime}$ that is essentially unchanged in the molecular frame when the material changes from the isotropic to any of the liquid crystalline phases.

The experiments to be reported here are schematically illustrated in Fig. 2. Oriented single domain liquid

(0)

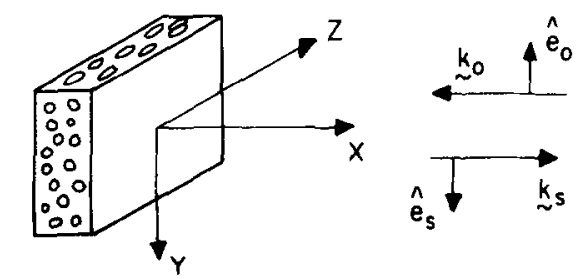

(i) $\hat{e}_{0}=\hat{z}, e_{s}=\hat{y}$ or $\hat{z}$
(ii) $\hat{e}_{0}=\hat{y}, e_{s}=\hat{y}$ or $\hat{z}$

(b)
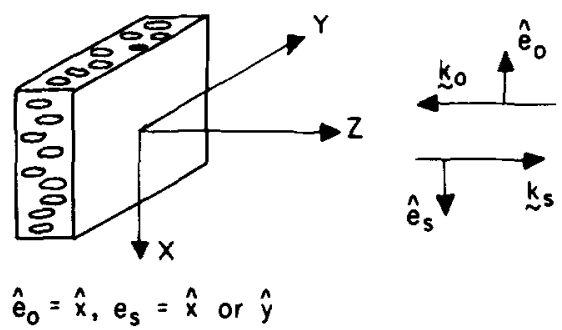

FIG. 2. Experimental geometries. (a) Optical axis of sample parallel to sample surface, (b) Optical axis of sample perpendicular to sample surface. 
crystalline samples are prepared in thin slabs between two glass slides. Figure 2(a) depicts the homogeneous orientation in which the uniaxial direction is parallel to the glass surfaces and Fig. 2(b) illustrates the homeotropic orientation in which the axis is normal to the glass surfaces. Incident laser light, wave vector $\mathbf{k}_{0}$ normal to the glass slides and polarization $\hat{e}_{0}$, is inelastically scattered and detected in the "back scattering geometry" with either of two polarizations. For a given geometry and incident polarization $\hat{i}=x, y, z$; the spectrally integrated intensities $I_{\hat{j} \mathfrak{i}}$ are measured for two polarizations $\hat{j}=x, y, z$. The primary measurements used in the subsequent analysis are the following three ratios of integrated intensities:

$$
R_{1}=I_{y z} / I_{z z}, R_{2}=I_{z y} / I_{y y}, \quad R_{3}=L_{y x} / I_{x x} \text {. }
$$

Lax and Nelson ${ }^{33}$ have developed theoretical expressions relating these observed intensity ratios to statistical averages of the Raman polarizability tensors $\alpha_{L}^{\prime}$ as expressed in the laboratory frame taking into account solid angle changes and transmission loss at the sample surfaces. For example, using the well known properties of irreducible tensor operators in the laboratory frame, the $\alpha_{L}^{\prime}$ for a particular vibration on a particular molecule are related to the $\alpha^{\prime}$ in the molecular frame by $^{11}$

$\alpha_{L}^{\prime}=\alpha(0,0) \hat{\boldsymbol{\beta}}_{0}^{(0)}+\sum_{m} \hat{\boldsymbol{\beta}}_{m}^{(2)} \sum_{m^{\prime}} \alpha\left(2, m^{\prime}\right) D_{m m^{\prime}}^{(2)}(\alpha, \beta, \gamma)$,

where the $\hat{\boldsymbol{\beta}}_{m}^{(2)}$ are the irreducible tensors of rank 2 and the $\alpha(2, m)$ are their coefficients in this representation, i.e.,

$$
\alpha^{\prime}=\alpha(0,0) \hat{\beta}_{0}^{(0)}+\sum_{m} \alpha(2, m) \hat{\beta}_{m}^{(2)} .
$$

Using the Cartesian form for the tensor components $\alpha_{i j}^{\prime}$ in the molecular frame, the components $\left(\alpha_{L}^{\prime}\right)_{i j}$ in the lab frame are related by the expressions in Appendix $I$.

The Lax-Nelson results for the present geometries are

$$
R_{1}=C_{n} r_{1}, R_{2}=r_{2} / C_{n}, R_{3}=r_{3},
$$

where the total correction factor $C_{n}$ is

$$
C_{n}=\left(\frac{n_{\text {out }}+\sqrt{\epsilon_{\text {gE }}}}{n_{\text {out }}+\sqrt{\epsilon_{x x}}}\right)^{2}
$$

and

$$
r_{1}=\frac{\left\langle\left(\alpha_{L}^{\prime}\right)_{y z}^{2}\right\rangle}{\left\langle\left(\alpha_{L}^{\prime}\right)_{x z}^{2}\right\rangle}, \quad r_{2}=\frac{\left\langle\left(\alpha_{L}^{\prime}\right)_{s y}^{2}\right\rangle}{\left\langle\left(\alpha_{L}^{\prime}\right)_{y y}^{2}\right\rangle}, \quad r_{3}=\frac{\left\langle\left(\alpha_{L}^{\prime}\right)_{y x}^{2}\right\rangle}{\left\langle\left(\alpha_{L}^{\prime}\right)_{x x}^{2}\right\rangle} .
$$

The objective now is to relate these three ratios to the orientational statistics of the liquid crystal molecule. Before proceeding, we should explicitly state an assumption that is tacitly made in almost all discussions of nematic order, namely, that the molecule has some one axis that is special or different from all others. Obviously, if one assumed the molecules to be cylindrical rods, the symmetry axis is this special axis. However, molecules are not really cylindrical rods and if such an axis exists, it more probably is related to the microscopic rotational motions of the molecule. That is, rotational correlation times about one particu- lar axis probably are much shorter than for any other. For whatever reason, we assume such a unique molecular axis. Note, however, we have not assumed at this point that the molecule has cylindrical symmetry about this axis. Thus, we define a particular "molecular reference frame" in which this unique axis defines the 3axis. Consider now any particular Raman active vibration. The Raman tensor for this vibration will have some form $\alpha_{m}^{\prime}$ in this molecular reference frame. However, there is always some other molecular axis, related to this one, in which the Raman tensor has the diagonal form

$$
\alpha_{d}^{\prime}=\alpha\left(\begin{array}{lll}
a & 0 & 0 \\
0 & b & 0 \\
0 & 0 & 1
\end{array}\right) .
$$

Using the equations in Appendix I, the components of $\alpha_{m}$ (in the molecular frame discussed above) can be expressed in terms of $\alpha, a, b$ and the three Eulerian angles $\alpha_{0}, \beta_{0}$, and $\gamma_{0}$ that bring the principal axis of $\alpha_{m}$ (that is, the axis for which $\alpha$ has the diagonal form) into coincidence with the special symmetry axis for the molecule. Obviously, there is some ambiguity in the choice of $\alpha_{0}$ and $\gamma_{0}$. However, this is associated with questions raised earlier concerning the assumption of local uniaxial symmetry and will prove to be unimportant for the present discussion.

For a system with macroscopic uniaxial symmetry, there are only four independent averages $\left\langle\alpha_{i j} \alpha_{k l}\right\rangle$. In terms of these, the three ratios $[\mathrm{Eq}$. (24)] can be written

$$
r_{1}=\frac{\left\langle\left(\alpha_{L}^{\prime}\right)_{x z}^{2}\right\rangle}{\left\langle\left(\alpha_{L}\right)_{z z}^{2}\right\rangle}, \quad r_{2}=\frac{\left\langle\left(\alpha_{L}^{\prime}\right)_{x z}^{2}\right\rangle}{\left\langle\left(\alpha_{L}^{\prime}\right)_{x x}^{2}\right\rangle}, \quad r_{3}=\frac{\left\langle\left(\alpha_{L}^{\prime}\right)_{x y}^{2}\right\rangle}{\left\langle\left(\alpha_{L}^{\prime}\right)_{x x}^{2}\right\rangle} .
$$

In fact these are the only independent ratios one can have for a system with uniaxial symmetry. Following the above discussion, these ratios can be expressed in terms of the microscopic molecular quantities $a$ and $b$, the Eulerian angles $\alpha_{0}, \beta_{0}$, and $\gamma_{0}$, and the orientational order parameters of $L=2,4$.

The simplest results are obtained for the case of cylindrical symmetry around the major molecular axis. In Appendix II we express the $\left\langle\left(\alpha_{L}^{\prime}\right)_{\alpha \beta}^{2}\right\rangle$ for a uniaxial phase consisting of molecules of arbitrary shape in terms of the order parameters and the components $\alpha_{i j}^{\prime}$ (relative to the fixed molecular frame), which are related to Eq. (25) through the Eulerian angles $\alpha_{0}, \beta_{0}$, and $\gamma_{0}$. The assumption of cylindrical symmetry about the 3 -axis obtains

$$
\begin{aligned}
A^{-2}\left\langle\left(\alpha_{L}^{\prime}\right)_{x x}^{2}\right\rangle= & \frac{1}{9}+\frac{3}{16} B+\frac{1}{4} C+\frac{1}{18} D+\frac{11}{288} D^{2}+\left(\frac{1}{8} B+\frac{1}{2} C-\frac{1}{8} D\right. \\
& \left.-\frac{5}{48} D^{2}\right)\left\langle\cos ^{2} \beta\right\rangle+\left(\frac{3}{16} B-\frac{3}{4} C+\frac{3}{32} D^{2}\right)\left\langle\cos ^{4} \beta\right\rangle, \\
A^{-2}\left\langle\left(\alpha_{L}^{\prime}\right)_{x y}^{2}\right\rangle= & \frac{1}{16} B+\frac{1}{4} C+\frac{1}{32} D^{2}+\left(\frac{3}{8} B-\frac{1}{16} D^{2}\right)\left\langle\cos ^{2} \beta\right\rangle \\
& +\left(\frac{1}{16} B-\frac{1}{4} C+\frac{1}{32} D^{2}\right)\left\langle\cos ^{4} \beta\right\rangle, \\
A^{-2}\left\langle\left(\alpha_{L}^{\prime}\right)_{x z}^{2}\right\rangle= & \frac{1}{4} B+\frac{1}{4} C-\left(\frac{3}{4} C-\frac{1}{8} D^{2}\right)\left\langle\cos ^{2} \beta\right\rangle \\
& -\left(\frac{1}{4} B-C+\frac{1}{8} D^{2}\right)\left\langle\cos ^{4} \beta\right\rangle, \\
A^{-2}\left\langle\left(\alpha_{L}^{\prime}\right)_{z z}^{2}\right\rangle= & +\frac{1}{9} B-\frac{1}{8} D+\frac{1}{36} D^{2}-\left(B-2 C-\frac{1}{3} D+\frac{1}{8} D^{2}\right\rangle \\
& \left\langle\cos ^{2} \beta\right\rangle+\left(\frac{1}{2} B-2 C+\frac{1}{4} D^{2}\right)\left\langle\cos ^{4} \beta\right\rangle,
\end{aligned}
$$


where

$$
\begin{aligned}
& A=\alpha_{11}^{\prime}+\alpha_{22}^{\prime}+\alpha_{33}^{\prime}, \\
& B=\left(1 / A^{2}\right)\left[\frac{1}{4}\left(\alpha_{11}^{\prime}-\alpha_{22}^{\prime}\right)^{2}+\alpha_{12}^{\prime 2}\right], \\
& C=\left(1 / A^{2}\right)\left(\alpha_{13}^{\prime 2}+\alpha_{23}^{\prime 2}\right), \\
& D=(1 / A)\left(2 \alpha_{33}^{\prime}-\alpha_{11}^{\prime}-\alpha_{22}^{\prime}\right) .
\end{aligned}
$$

At a particular temperature the three ratios in Eq. (26) give three equations linear in the two unknowns $\left\langle\cos ^{2} \beta\right\rangle$ and $\left\langle\cos ^{4} \beta\right\rangle$ with three parameters $B, C$, and $D$. In addition, the depolarization ratio measured in the isotropic phase obtains another relation between these three parameters,

$$
\begin{aligned}
& R_{\text {iso }}=\frac{3}{4} \frac{12 B+12 C+D^{2}}{5+12 B+12 C+D^{2}}, \\
& R_{180}=\frac{3\left(a^{2}+b^{2}+1-a-b-a b\right)}{5(a+b+1)^{2}+4\left(a^{2}+b^{2}+1-a-b-a b\right)} .
\end{aligned}
$$

If the type of vibration is identified and the relative orientation $\Omega_{0}\left(\alpha_{0}, \beta_{0}, \gamma_{0}\right)$ is known, we can solve from the data for the two order parameters

$$
\begin{aligned}
& A_{0}^{(2)}=\frac{1}{2}\left\langle 3 \cos ^{2} \beta-1\right\rangle, \\
& A_{0}^{(4)}=\frac{1}{8}\left\langle 35 \cos ^{4} \beta-30 \cos ^{2} \beta+3\right\rangle .
\end{aligned}
$$

Consider two special cases. First, if the vibration is known to have uniaxial symmetry in the local frame and the symmetry axis makes an angle $\beta_{0}$ with the major molecular axis, the 3-axis, we have $a=b$ in Eq. (25) and

$$
\begin{aligned}
& A=1+2 a, \quad B=\left(1 / 4 A^{2}\right)(1-a)^{2} \sin ^{4} \beta_{0}, \\
& C=\frac{1}{A^{2}}(1-a)^{2} \sin ^{2} \beta_{0} \cos ^{2} \beta_{0}, \quad D=\frac{1}{A}(1-a)\left(3 \cos ^{2} \beta_{0}-1\right) .
\end{aligned}
$$

The depolarization ratio in the isotropic phase,

$$
R_{\mathrm{i}=0}=(1-a)^{2} /\left(3+4 a+8 a^{2}\right),
$$

determines the parameter $a$. This particular case is of special interest since there are four independent measurements $R_{1}, R_{2}, R_{3}$, and $R_{1 \mathrm{so}}$ and only four quantities to be deduced, $A_{0}^{(2)}, A_{0}^{(4)}, a$, and $\beta_{0}$. Furthermore, if the angle $\beta_{0}$ is known, from molecular models or otherwise, the problem is overdetermined.

For the second case, suppose the molecular polarizability is not uniaxial $(a \neq b)$ and choose the $\hat{1} \hat{3}$-molecular plane to include the principal axis for $\alpha_{d}$. In place of Eqs. (31) we obtain

$$
\begin{aligned}
& A=1+a+b, \\
& B=\left(1 / 4 A^{2}\right)\left[a-b+(1-a) \sin ^{2} \beta_{0}\right], \\
& C=\left(1 / A^{2}\right)(1-a)^{2} \sin ^{2} \beta_{0} \cos ^{2} \beta_{0}, \\
& D=(1 / A)\left[2-(a+b)-3(1-a) \sin ^{2} \beta_{0}\right] .
\end{aligned}
$$

Using $R_{180}$ [Eq. (29)] and the three other ratios, it is possible to solve, for example, for $A_{0}^{(2)}, A_{0}^{(4)}, a$, and $b$ at some temperature with $\beta_{0}$ being treated as an unknown parameter. The same measurements can be made at different temperatures and compared. If the assumptions discussed above are correct, the deduced values of $a, b$, and $\beta_{0}$ must be independent of temperature. (a) MBBA

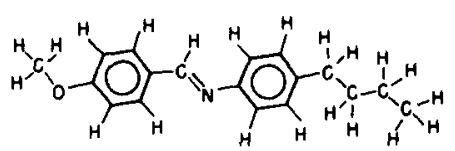

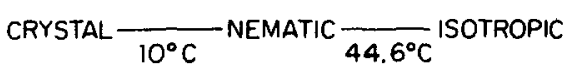

(b) BBCA

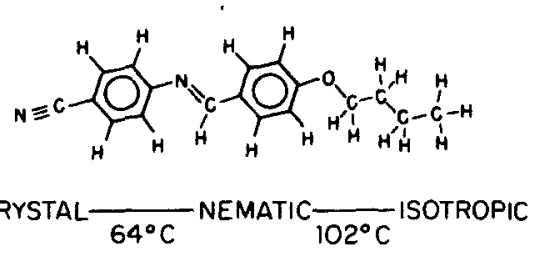

(c)

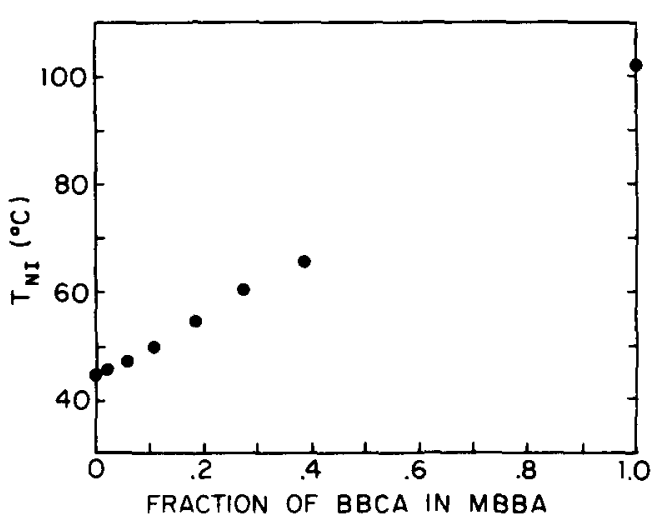

FIG. 3. Schematic diagram of molecular structures of (a) MBBA, (b) BBCA, and a phase diagram of mixtures of these two compounds (c).

This paper will proceed by assuming cylindrical local symmetry about some molecular axis, designated as the 3-axis. Values of $A_{0}^{(2)}, A_{0}^{(4)}, a, b$, and $\beta_{0}$ will be deduced for several vibrations and the sensitivity of the results to reasonable deviations from cylindrical local symmetry will be examined. We will show that the results are insensitive to such deviations.

\section{EXPERIMENTAL}

\section{A. Materials}

\section{Properties of samples}

The initial objective of this work was to investigate the nematic phase of the extensively studied room temperature liquid crystal compound $N-\left(p^{\prime}-\right.$ methoxybenzylidene)- $p$ - $n$-butylaniline (MBBA) by examining the Raman spectrum of a probe molecule, $N$ - ( $p$ '-butoxybenzylidene)- $p$-cyanoaniline (BBCA). BBCA was chosen because of its structural similarity to the MBBA host and also because of its terminal cyano group whose bond axis is nearly parallel to the major axis of the molecule. Associated with the stretching vibration of the cyano group there is a strong, narrow, anisotropic Raman band which is spectrally isolated from all the other Raman bands of the BBCA and MBBA molecules and thus ideally suited to the study of long range orientational order.

Figure 3 illustrates the molecular structures, se- 
(0) 40.8

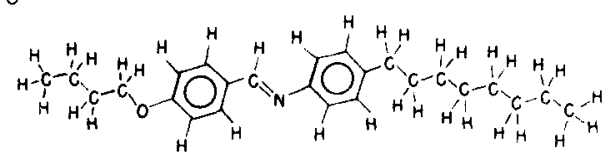

CRYSTAL $-37^{\circ} \mathrm{C}$ SMECTIC $8 \frac{}{48.8^{\circ} \mathrm{C}}$ SMECTIC A $\frac{}{63.7^{\circ} \mathrm{C}}$ NEMATIC $\frac{}{78.5^{\circ} \mathrm{C}}$ ISOTROPIC

(b)

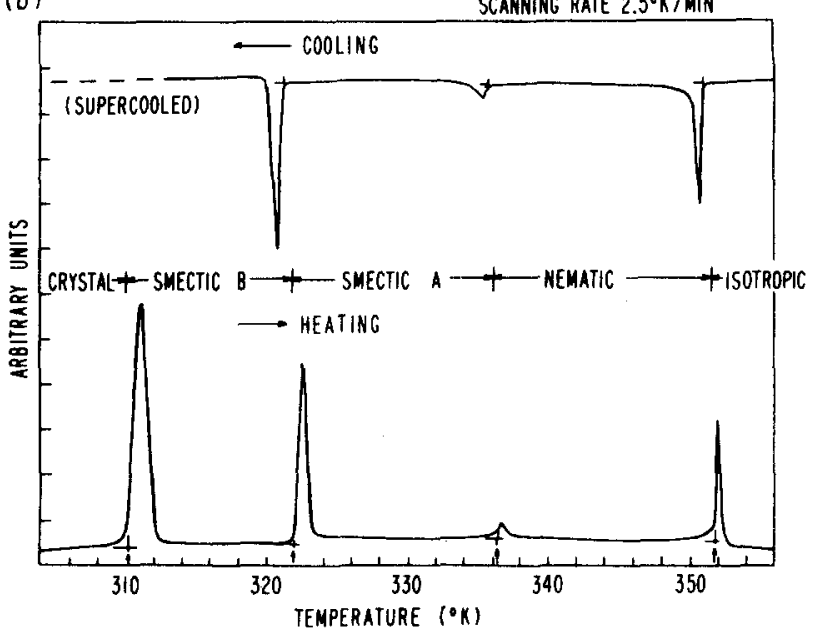

FIG. 4. (a) Molecular structure and sequence of mesophases, and (b) calorimetry trace of compound 40.8 .

quences of respective phases, and a portion of the phase diagram for mixtures of $\mathrm{MBBA}$ and $\mathrm{BBCA}$. Both molecules are Schiff's bases with approximately the same molecular dimensions and both exhibit a single nematic mesophase between their isotropic liquid and crystalline phases. BBCA is readily soluble in MBBA for concentrations up to $30 \%$ by weight ${ }^{34}$ at room temperature. Our principal source of materials has been the Harvard Chemistry Laboratory ${ }^{35}$ but, on occasion, commercially available MBBA from Vari-Light Corp. ${ }^{36}$ has also been used.

In order to minimize the perturbing influence of the $\mathrm{BBCA}$ guest on the physical properties of the MBBA host, the concentration of BBCA was kept as small as possible commensurate with reasonable signal-to-noise in the Raman spectrum. In practice, it was found that $20 \% \mathrm{BBCA}$ in MBBA was a suitable compromise concentration. Phase separation was not a problem at this concentration, although it would become bothersome at higher concentrations. For example, the coexistence range for a $40 \%$ mixture was found to be $\sim 1{ }^{\circ} \mathrm{C}$.

Some effort was made to investigate other liquid crystals using probe molecules, but this was not found to be very fruitful. We chose rather to dispose of the probe molecule completely and concentrate on the natural Raman modes of the neat MBBA. This will be discussed further below and it will be seen that for the $20 \%$ mixture of $\mathrm{BBCA}$ in $\mathrm{MBBA}$ the values of $A_{0}^{(2)}$ and $A_{0}^{(4)}$ deduced from the Raman measurements on the $\mathrm{BBCA}$ cyano vibration are indentical to the results deduced from the vibrational modes of pure MBBA.
We also examined another Schiff's base liquid crystal, $N$ - ( $p^{\prime}$-butoxybenzylidene)- $p-n$-oxtylaniline (BBOA) (or 40.8 in Verbit's line notation ${ }^{37}$ ), which has been identified as having nematic, and smectic $A$ and $B$ phases. ${ }^{38}$ The molecular structure and phase sequences for 40.8 are shown in Fig. 4(a). Figure $4(\mathrm{~b})$ is a calorimetric trace of 40.8 obtained using a Perkin-Elmer differential scanning calorimeter (DSC-2) calibrated against an indium standard for both temperature and thermal responses. The various transition temperatures from the DSC agree with those observed by optical microscopy. All transitions are first order and we estimate their latent heats to be $3.185,1.303,0.229$, and $0.919 \mathrm{cal} / \mathrm{g}$ for the crystal-to-smectic $B$, smectic $B$-to-smectic $A$, smectic $A$-to-nematic, and nematic-toisotropic transitions, respectively. The uncertainty in these values is estimated to be $\pm 0.009 \mathrm{cal} / \mathrm{g} .{ }^{39}$ The small latent heat between the smectic $\mathrm{A}$ and nematic phases is of interest and we will show in the next section that the index of refraction data also show a very nearly continuous change through this transition.

Liquid crystal materials having the Schiff's base central linkage are notorious for their poor chemical stability, owing to the ease with which they are hydrolyzed. All reasonable precautions have been taken to retard the degradation of our samples, though it was never completely eliminated.

\section{Alignment of samples}

Samples of liquid crystal materials were sandwiched between two $1 \frac{1}{2} \times 1 \times 0.04 \mathrm{in}$. microscope slides separated by Teflon spacers ranging from 25 to $250 \mu \mathrm{m}$ in thickness, as shown in Fig. 5(a). Prior to assembling

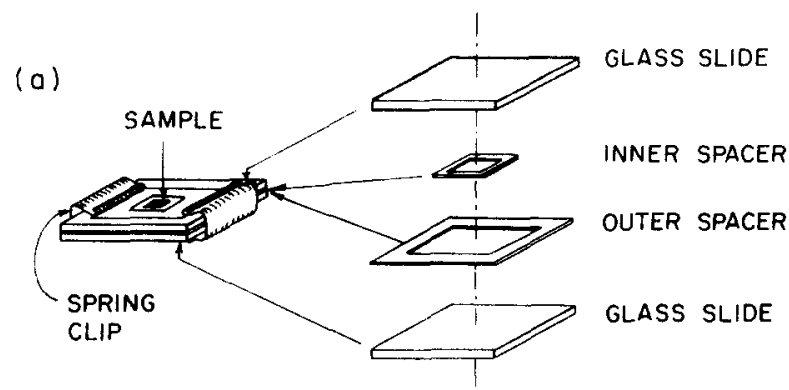

(b)

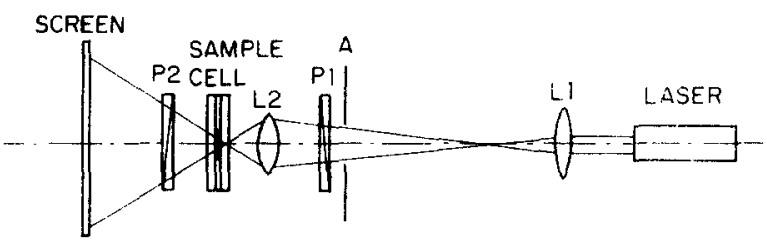

(c)
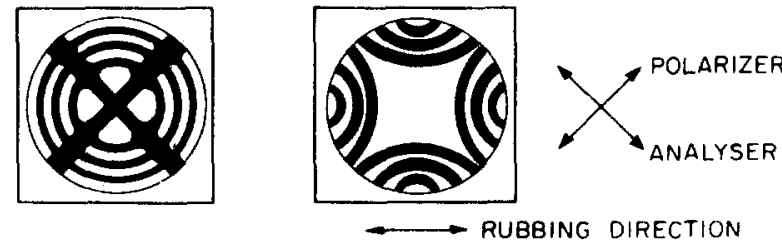

FIG. 5. (a) Sample cell, (b) arrangement for examining the conoscopic figures, and (c) the interference fringes for homeotropic (left) and homogeneous (right) alignment. 
the cells, the slides and spacers were carefully cleaned and baked at temperatures greater than $100{ }^{\circ} \mathrm{C}$. Homeotropic (perpendicular) alignment was achieved by coating the cleaned slides with a surfactant such as hexadecyltrimethylammoniumbromide (HTAB). With some practice, it was possible to obtain satisfactory and reproducible homogeneous (parallel) alignment by simple unidirectional rubbing of the cleaned slides. The cells were drop-filled, using a hot plate when necessary (e.g., for the 40.8), and clamped on two sides with spring clips. Homeotropic and homogeneous smectic $A$ samples were obtained by slow cooling from the respective nematic orientation.

The alignment of the samples was routinely checked in the following way. First, the cells were inspected with naked, though well trained, eyes for any gross defects. Having passed this visual test, cells were observed under an optical microscope, allowing the alignment to be examined over distances as small as $20 \mu \mathrm{m}$. The conoscopic figure obtained by normal illumination of the samples between two crossed polarizers with convergent light [Fig. 5(b)] provided a third way of confirming the alignment. Typical in terference patterns show a dark cross with concentric dark circles and families of hyperbolae like fringes centered at the axis of the beam for homeotropic and homogeneous alignment, respectively. ${ }^{40}$ The geometrical appearance of these patterns, for example, their centering, their symmetry, etc., unambiguously reflect the success of the alignment. By shifting the focal plane along the direction of the beam, or moving the sample cell perpendicular to the beam, we could detect misaligned areas as big as the whole sample and as small as $20 \mu \mathrm{m}$ across.

After determining that the alignment was satisfactory, the transition temperatures of the material in the cell were checked using the hot stage under an optical microscope. The absence of any initial drop in transition temperatures, particularly $T_{N I}$, was taken as evidence that the process of alignment did not contribute appreciably to the deterioration of the materials.

After determining that the alignment was satisfactory, the transition temperatures of the material in the cell were checked using the hot stage under an optical microscope. The absence of any initial drop in transition temperatures, particularly $T_{N I}$, was taken as evidence that the process of alignment did not contribute appreciably to the deterioration of the materials.

There are two factors limiting the thickness of the samples. The first is naturally how much the boundary conditions imposed by the aligning techniques can force the material in the bulk to follow the alignment. By experience samples up to $300 \mu$ thick, which is by no means the upper limit, always align reasonably well. The second factor is the Raman scattering measurement itself. The data depend strongly upon the thickness in the nematic phase even for samples as thin as $30 \mu$. This is attributed to the turbidity and depolarized forward scattering by the long-range director fluctuations. The thickness of the sample is thus an important parameter in the experiments and was measured under a mi- croscope by focusing the objective on the inner surfaces of the glass slides illuminated with dark bakcground. For each sample, measurements were made on the four outside corners of the inner spacer where there was no liquid crystal present, and no systematic deviation was observed among the four sets of readings. The thickness at the center, where the light scattering experiment was performed, was taken as the average of these readings. At a magnification of $\times 160$, the thickness could be determined with an uncertainty of $\pm 2 \mu \mathrm{m}$.

\section{B. Raman spectroscopy system}

The laser Raman spectroscopy system consists of three main components: an argon ion laser and associiated optics, a spectrometer with digital data acquisition devices, and a temperature controlled oven that houses the sample cell.

A Coherent Radiation model 52-B $3 \mathrm{~W}$ c.w. argon ion laser operating at $5145 \AA$ was the exciting source. Occasionally, the $4800 \stackrel{A}{A}$ line was used to check the spectra. The laser output was stabilized using a servo system similar to the commercially available Coherent Radiation 235 light regulator. Long term (periods of several hours) drifts in the laser intensity were thus kept below $5 \%$ and short term drifts (periods of $30 \mathrm{~min}$ ) were even smaller. The experiments were done with less than $100 \mathrm{~mW}$ incident power, monitored continuously by a Spectra-Physics $401 \mathrm{C}$ powermeter which viewed a small amount of light deflected from a beam splitter. The illuminated sample area is estimated to be on the order of $200 \mu \mathrm{m}$ in diameter. The scattered light is imaged onto the entrance slit of a SPEX 1400-II double monochromator equipped with two 600 groves $/ \mathrm{mm}$ gratings blazed at $5000 \AA$ in second order. A polaroid HN32 film polarizer is used to analyze the polarization state of the scattered light and an oriented quartz wedge polarization scrambler between the polaroid and the entrance slit serves to eliminate the polarization dependence of the spectrometer transfer characteristic. Light emerging from the exit slit of the spectrometer is detected by a cooled EMI 9558 photomultiplier tube with an S-20 cathode response. The output pulses from the PMT are amplified and fed via a discriminator into the memory of an RIDL Nuclear Chicago model 34-12B 400 channel analyzer. The stored data can be displayed on a CRT on the multichannel analyzer or plotted on an $X-Y$ recorder.

\section{Raman scattering and treatment of data}

To establish the geometries mentioned in the last section (Fig. 2) we rotated either the sample cell (the constant temperature housing) or the polarization of the incoming beam (with a piece of half-wave plate cut for $5145 \AA$ ). The optical axis of a homogeneous sample was oriented relative to the direction of the incident polarization by minimizing the transmission through a crossed polarizer placed behind the sample. For example, when the optical axis is strictly parallel or perpendicular to the fixed direction of the incident polarization, the transmission is at a minimum with a crossed polarizer after the sample. In the nematic 
phase the extinction was less perfect for thick samples because of the depolarized forward scattering; nevertheless, a minimum could be reached when the sample was properly oriented.

The transition temperature was checked during the experiment either by moving the sample to the microscope hot stage, or with the temperature measuring device in the oven itself. For the latter, because the front face of the sample cell was partially in contact with the room atmosphere, we could observe the temperature gradient across the circle where the beam enters. The nematic-to-isotropic transition was determined by the change of the transmission pattern of the beam as well as by the appearance of the sample viewed with naked eye from outside. The difference between the temperature readings from the thermistor inside the oven block and the known temperature for the transitions occurring at the center of the circle and near the circumference was less than $0.05^{\circ} \mathrm{C}$ for samples of 40.8. Similar differences were considerably smaller for the nematic samples with much lower $T_{N I}$. The same observation can also be made at the smectic $A$-tonematic transition.

We also observed laser heating of the samples, particularly near the nematic-to-isotropic transition where thermal lensing accompanied the growth of the transmission spot when the laser was first incident on the sample. The region under illumination undergoes a transition before the temperature of the oven reaches $T_{N I}$ and a "hole" can be seen on the sample which is "burnt" by the beam. For the nematic samples of the mixtures or neat MBBA, an incident intensity of $80 \mathrm{~mW}$ "lowered" the transition by roughly $1.5^{\circ} \mathrm{C}$. For samples of 40.8 the effect was less serious and with $50 \mathrm{~mW}$ incident power the apparent decrease in $T_{N I}$ was less than $0.2^{\circ} \mathrm{C}$ This can be attributed to different absorption at the incident wavelength as evidenced by the yellowish appearance of the MBBA and the nearly colorless appearance of 40.8 .

The spectrometer is capable of yielding Raman spectra of typical resolution as high as several tenths of a $\mathrm{cm}^{-1}$ by reducing the slit width. However, in order to make use of the solid angle correction formulas it is simpler to have a fixed collecting solid angle, determined by an aperture after the collecting lens, and the entire area under illumination imaged into the slit. Although this means poor resolution, it does not affect the intensity ratio measurements, since the observed line shape $I_{\text {obs }}(\omega)$ is a convolution of the slit function $S\left(\omega^{\prime}\right)$ with the true line shape of the power spectrum $I(\omega)$,

$$
I_{\text {obs }}(\omega)=\int_{-\infty}^{\infty} d \omega^{\prime} S\left(\omega^{\prime}\right) I\left(\omega+\omega^{\prime}\right) .
$$

It is seen that the integrated intensity of the observed line shape is proportional to that of the true line shape with a factor depending on the slit function only. Therefore, for a well isolated Raman band, such as the cyano stretching vibration, the depolarization ratios are obtained by the ratios of the area under the observed line profiles. This is done with a planimeter.

The situation is more complicated when the bands are not well resolved, since it is necessary then to devise some method for obtaining the area under each band separately. In principle, this can be done with a numerical fitting of the observed composite band profile. We assume a triangular slit function and Lorentzian line shapes of different widths and peak heights and do the fitting on an HP9820 calculator. Depending on the situation, a number of Lorentzians are fed in to fit the observed band profiles. The fitting turns out to be satisfactory and the areas are easily calculated from the best-fit parameters for each band. We observe, however, for each specific band of interest in a particular scattering geometry, the linewidths of depolarized and polarized spectra do not differ appreciably at a given temperature. This makes possible a simplification in the analysis of the data. For bands that can be approximated by Lorentzians of nearly the same widths, the peak heights are themselves proportional to the integrated intensities with the same proportionality factors. This is indeed confirmed by comparing the results obtained in these two different ways. We thus measure the depolarization ratios for bands in a composite profile by comparing the peak heights of depolarized and polarized spectra and estimate that the error introduced by so doing is less than $4 \%$.

\section{Measurement of indices of refraction}

Index of refraction data are required for the solid angle and transmission loss corrections. ${ }^{33}$ They also provide results on the order parameter which can be compared with the Raman measurements. The measurements are conveniently made with a Pulfrich refractometer (Bellingham and Stanley, Inc.) on which the sample is aligned homeotropically between the top surface of the prism and a piece of fused quartz cover glass. Measurements were made at $5145 \AA$ (argon laser) and $6328 \AA$ (He-Ne laser), and the refractive indices at the wavelength of the Raman scattered light were found by interpolation.

Further experimental details are available in the Ph. D. thesis of Jen. ${ }^{41}$

\section{STUDY OF THE CYANO STRETCHING VIBRATION}

\section{A. General observations and data}

The vibrational Raman band associated with the stretching mode of the cyano group is located at 2225 $\mathrm{cm}^{-1}$, corresponding to scattered radiation at $5810 \breve{\mathrm{A}}$

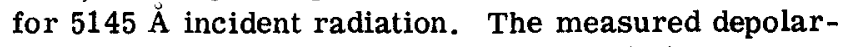
ization ratios $R_{1}$ and $R_{2}$, defined in Eq. (19), show strong dependence on sample thickness (see Fig. 6). This effect is quite reproducible and occurs for the other Raman bands we have studied (see below), as well as for the $C N$ stretching band. There are two ways such a thickness dependence might arise. First, if the director were not uniform over the scattering volume, due to disclinations, large impurity particles, etc., one might expect to observe a thickness dependence. This seems improbable in the present case considering the reproducibility of the data and also considering the fact that the data are insensitive to the application of 
static magnetic fields of up to $5.6 \mathrm{kG}$ along the direction of rubbing (Fig. 7). We thus believe the observed thickness dependence is attributable to the second possibility, namely, multiple scattering by long wavelength director fluctuations, which causes spurious depolarizations, which causes spurious depolarization of both the incident and scattered beams. Thus, the thicker the sample the greater is the "scrambling" effect. This trend is clearly evident in our data, and it is not surprising that previous studies of the intensity and/or depolarization ratios of various Raman bands of nematic materials have failed to show any temperature dependence over the entire nematic range. ${ }^{42,43}$ Since theoretical correction for this multiple scattering effect is not simple and the data show a strong effect even at thicknesses as small as $30 \mu \mathrm{m}$, which is the thinnest sample we can use and still have satisfactory signal-tonoise ratios, we account for the effect by measuring the ratios $R_{1}$ and $R_{2}$ as functions of thickness and extrapolating to zero thickness.

Figure 6 shows the ratios $R_{1}$ and $R_{2}$ for a $20 \%$ mixture of BBCA in MBBA plotted against $\Delta T_{N T}-T$, at two thicknesses, together with the extrapolated values. The depolarization ratio in the isotropic phase is also shown, and is seen to be independent of thickness. This ratio $R_{\text {iso }}$ was also measured at various concentrations below
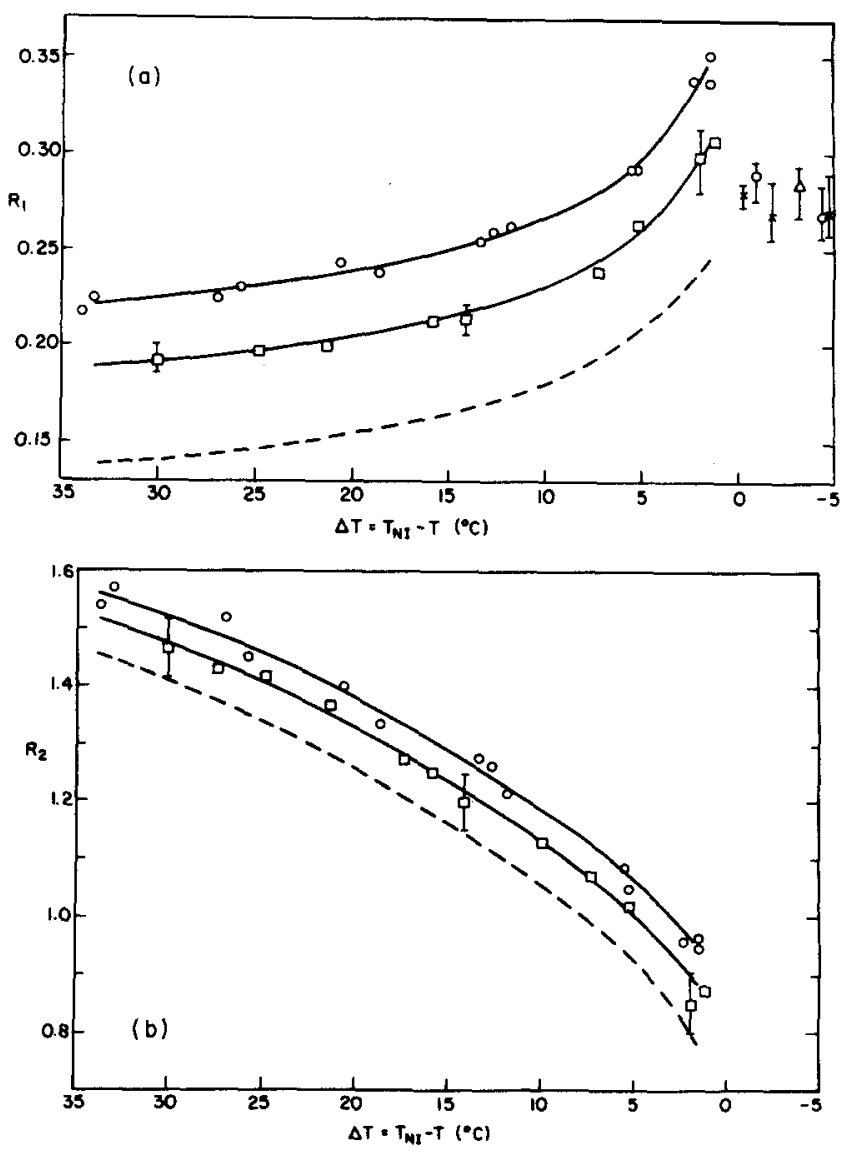

FIG. 6. Raman depolarization ratios $R_{1}$ (a) and $R_{2}$ (b) of the cyano stretching band for $20 \% \mathrm{BBCA}$ in $\mathrm{MBBA}$ at 29.3 (squares) and $54.5 \mu$ (circles) thick. Dashed lines are extrapolated data at zero thickness. For $R_{180}$ in (a) the triangle and the crosses are data from 125 and $250 \mu$ thick samples.

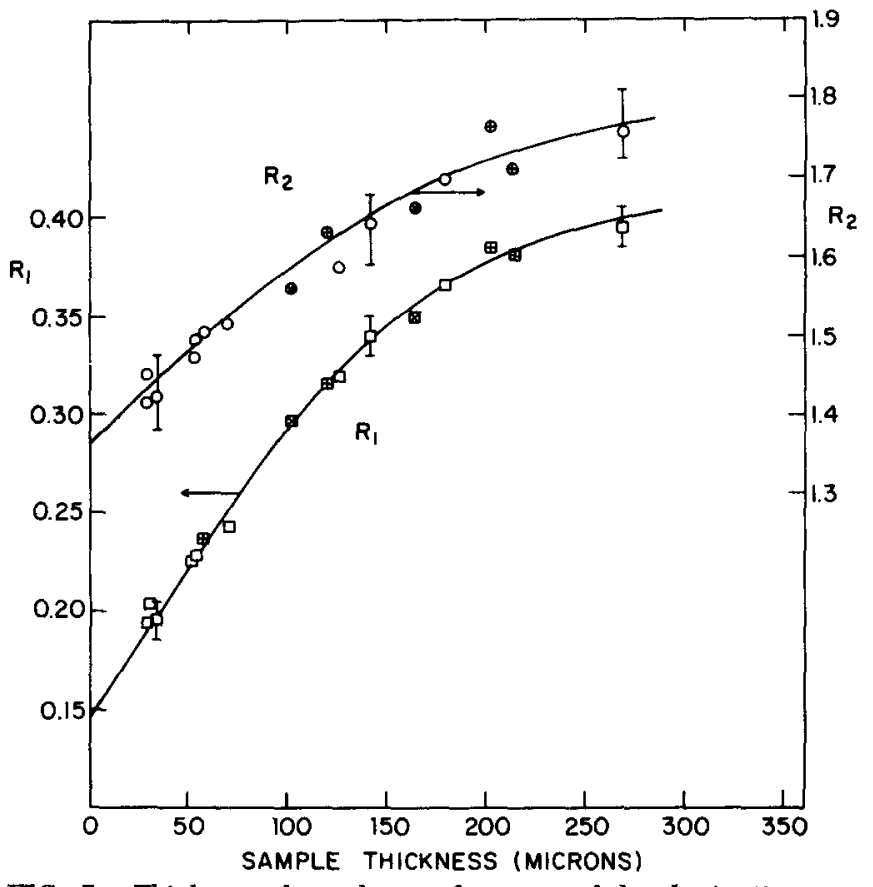

FTG. 7. Thickness dependence of measured depolarization ratios $R_{1}$ (squares) and $R_{2}$ (circles) of the cyano stretching band from samples of $20 \% \mathrm{BBCA}$ in MBBA at $27.0^{\circ} \mathrm{C}$ below transition. Data points with + and $x$ are respectively those with static magnetic field of 5.6 and $0.9 \mathrm{kG}$ along the direction of rubbing. $T_{N I}$ ranges from 54.5 to $56.5^{\circ} \mathrm{C}$.

$20 \%$ and no dependence on either temperature, within a range of $\sim 10^{\circ} \mathrm{C}$, or concentration was observed.

\section{B. Order parameters of the $20 \%$ mixture}

The Raman polarizability tensor for the localized cyano stretching vibration of the BBCA molecule has the uniaxial form of Eq. (25) with $a=b$. From the measured ratio $R_{1 \mathrm{so}}$ and $\mathrm{Eq}$. (32) the parameter $a$ was determined to be $0.055 \pm 0.020$. The surface coupling factor $C_{n}$ in Eq. (23) was calculated for the scattered light at $5810 \mathrm{~A}$, at each temperature, using linearly interpolated values obtained from the measured indices of refraction for the liquid crystal (Fig. 8) and the glass slides, which gives $n_{\text {out }}=n_{c}=1.5150$ with negligible temperature dependence. From a space-filled model for the $\mathrm{BBCA}$ molecule the angle $\beta_{0}$ between the symmetry axis of the cyano group and the major molecular axis is estimated to be $10^{\circ} \pm 2^{\circ}$. We thus obtained the order parameters $A_{0}^{(2)}$ and $A_{0}^{(4)}$, under the assumption of cylindrical molecular symmetry, from each pair of $R_{1}$ and $R_{2}$ at respective temperature by Eqs. (27), (30), and (31). These results are shown in Fig. 9. The uncertainties shown are estimated ${ }^{44}$ by taking into account of all the statistical uncertainties from $R_{1}, R_{2}, R_{\text {iso }}$ (or $a$ ), and $\beta_{0}$. The uncertainties due to the indices of refraction are negligibly small.

Although the MBBA and BBCA molecules are very similar in many respects, the order parameters obtained at such a high concentration are not necessarily those of neat MBBA. However, the available evidence supports the idea that $A_{0}^{(2)}$ obtained from the $20 \% \mathrm{mix}-$ ture is identical to that for MBBA, within experimental 


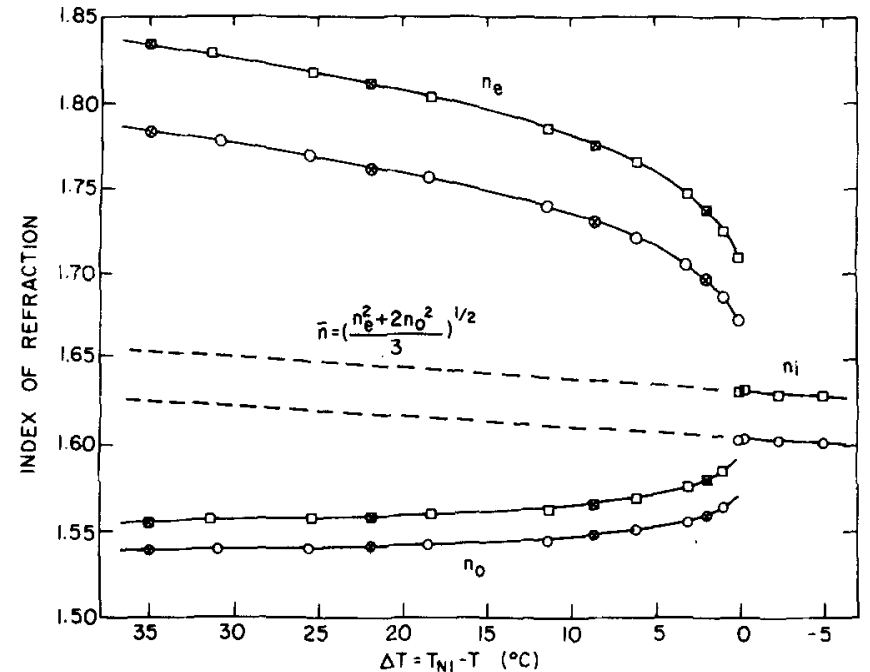

FIG. 8. Indices of refraction of $20 \% \mathrm{BBCA}$ in MBBA. Squares are measured at $5145 \AA$, and circles at $6328 \AA .\left(T_{N I}=55.25^{\circ} \mathrm{C}\right.$ for crossed data points, $T_{N I}=53.78^{\circ} \mathrm{C}$ for open points),

uncertainty. Proton NMR splittings (Fig. 10), diamagnetic anisotropies (Fig. 11), and optical indices of refraction (Figs. 8 and 12) for neat MBBA and the 20\% mixture are nearly identical. Since we assume here that the molecules are rigid cylinders, the macroscopic

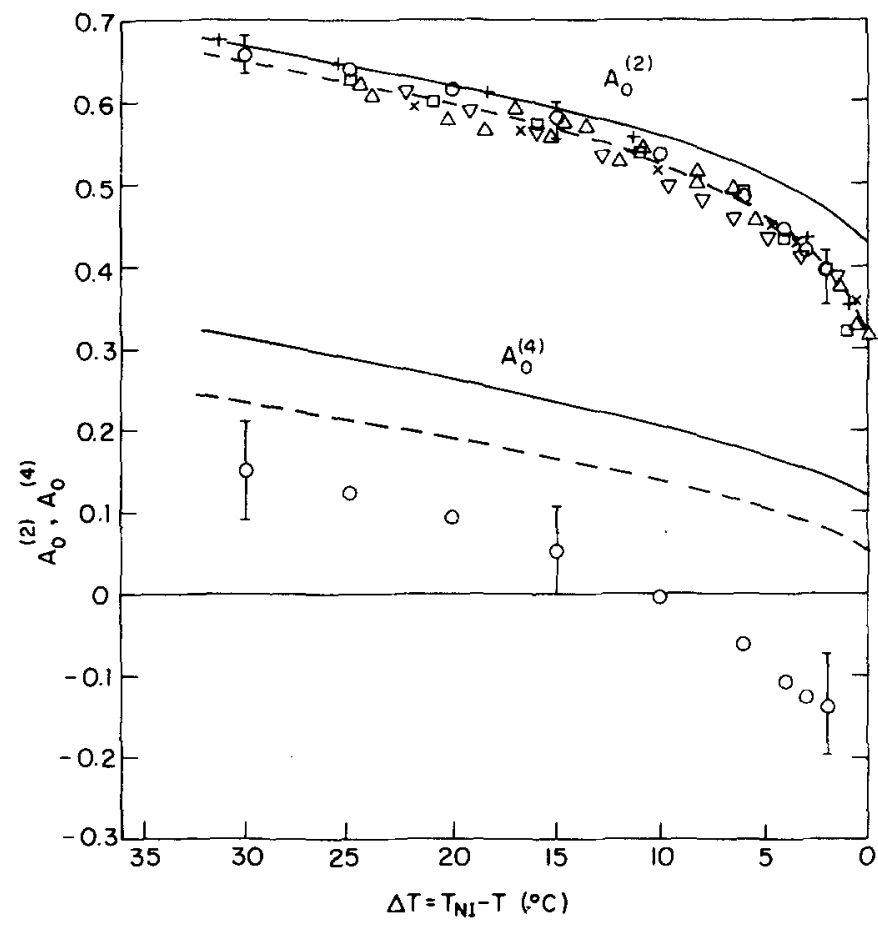

FIG. 9. Theoretical and experimental values of the nematic order parameters $A_{0}^{(2)}$ and $A_{0}^{(4)}$ of $20 \% \mathrm{BBCA}$ in MBBA. Solid lines: Maier-Saupe theory; dashed lines: Humphries-JamesLuckhurst theory with $\lambda=-0.55$ and $m=10 \pm 2$. 0 : Raman measurements; $\square$ : NMR from Ref. 24(b); crosses: relative measurements from optical dielectric anisotropy $(+: 20 \% \mathrm{mix}-$ ture; $\mathrm{x}$ : neat $\mathrm{MBBA})$; triangles: relative measurements from diamagnetic anisotropy ( $\Delta$ : from Ref. 18(c), $\Delta$ : from I. Haller, J. Chem. Phys. 57, 1400 (1972), $\nabla$ : Ref. 50.

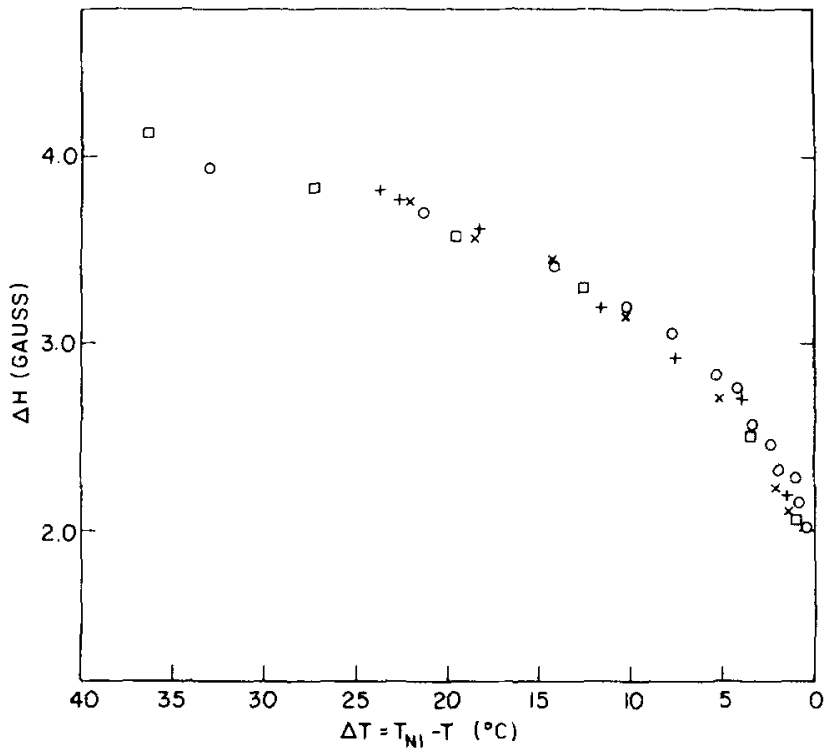

FIG. 10. Proton NMR splittings of MBBA $10: T_{N I}=45.0^{\circ} \mathrm{C}$, from P. Papon and J. -P. Lepesant, Chem. Phys. Lett. 12, $\left.331(1971) ;+: T_{N I}=46.64^{\circ} \mathrm{C} ; \mathrm{x}: T_{N I}=42.70^{\circ} \mathrm{C}\right]$ and $20 \% \mathrm{BBCA}$ in MBBA [ $\mathrm{a}: \mathrm{T}_{N I}=55.84^{\circ} \mathrm{C}$.

$Q_{x z}$, the semiempirical $Q_{z z}^{\prime}$, and the microscopic $A_{0}^{(2)}$ are all equivalent. Figure 9 shows a comparison of the data for $A_{0}^{(2)}$, from NMR measurements of partially deuterated $\mathrm{MBBA}^{24 b}$ and our Raman measurements, with $Q_{E z}$ and $Q_{z \varepsilon}^{\prime}$. Relative values of $A_{0}^{(2)}$ for the latter were obtained by assuming $A_{0}^{(2)}$ to be proportional to $\Delta \chi$ and $\Delta \alpha$ and fixing the proportionality factor to obtain agreement at $\Delta T=2{ }^{\circ} \mathrm{C}$. The temperature dependence and the absolute values for $A_{0}^{(2)}$ from the Raman data agree very well with those obtained from the other independent measurements.

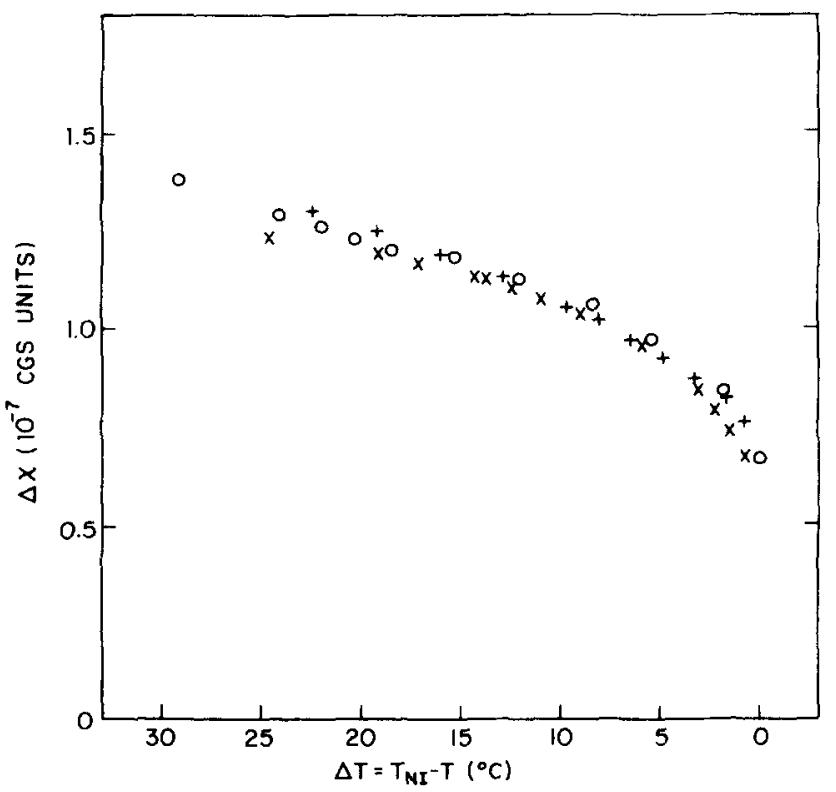

FIG. 11. Diamagnetic anisotropy of MBBA [O: from G. Sigaud and H. Gasparoux, J. Chim. Phys, 70, $699(1973), T_{N I}=$ 46. $0^{\circ} \mathrm{C}$; $\mathrm{x}$ : from I. Haller, J. Chem. Phys. 57, 1400 (1972), $T_{N I}=41.3-45.7^{\circ} \mathrm{C}$; + : from Ref. $49, T_{N I}=45.75^{\circ} \mathrm{C}$. 


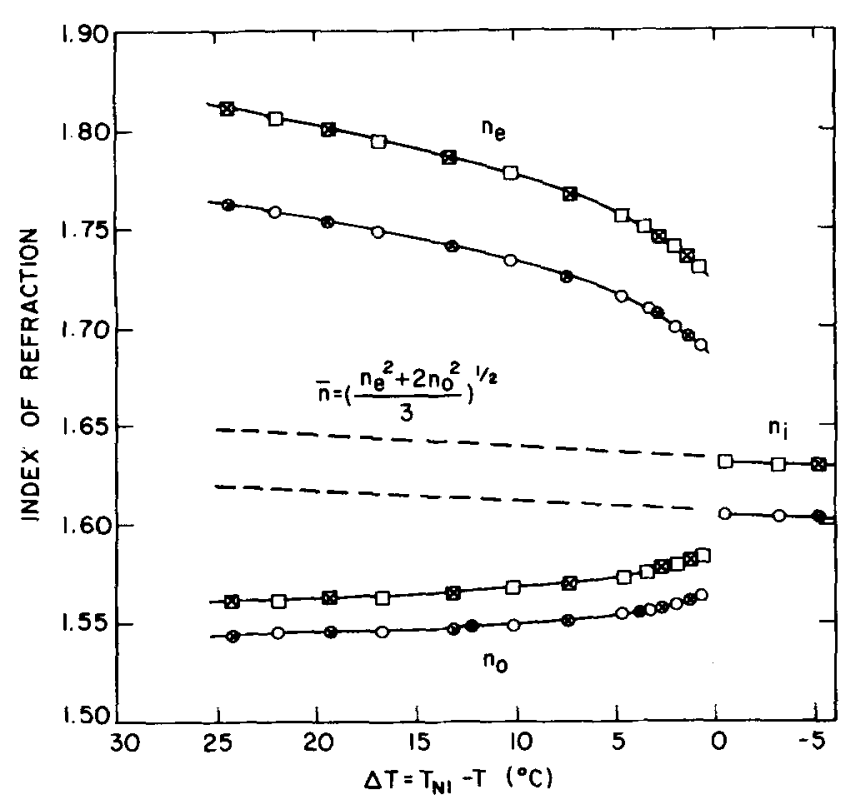

FTG. 12. Indices of refraction of MBBA versus reduced temperature at $5145 \AA\left[\square: T_{N I}=44.65^{\circ} \mathrm{C}, \otimes: T_{N I}=46.25^{\circ} \mathrm{C}\right]$ and at $6328 \AA\left[0: T_{N I}=44.65^{\circ} \mathrm{C}, \infty: T_{N I}=46.25^{\circ} \mathrm{C}, \bullet\right.$ : from $I_{\text {. Haller, }}$ H. A. Huggins, and M. J. Freiser, Mol. Cryst. Liquid Cryst. $\left.16,53(1972), T_{N I}=47.0^{\circ} \mathrm{C}\right]$.

\section{Remarks on the data analysis}

Before comparing our data with existing theories, two areas must be considered at greater length, since they have been tacitly assumed unimportant in the above discussion but could in principle affect our results.

(1) Director fluctuations. The order parameters $A_{0}^{(2)}$ and $A_{0}^{(4)}$ are measured relative to a fixed external laboratory axis while theoretical calculations of these quantities are made relative to the local director $\hat{n}(\mathbf{r})$. These two need not be identical and indeed will not be if the director deviates from the external symmetry axis. One mechanis $m$ that produces such deviations is thermal fluctuations of the director, and the effect of such fluctuations on the measured order parameters has been considered in detail by Sheng. ${ }^{45}$ He showed that, for small amplitude fluctuations, the probability distribution for the director can be approximated by a Gaussian,

$$
F(\hat{n}) \propto \exp \left(-\theta^{2} / \theta_{0}^{2}\right),
$$

where $\theta$ is the polar angle between the director and the $z$ axis of the laboratory frame and $\theta_{0}$ is the rms fluctuation of the director in the laboratory frame. Sheng expressed his results in terms of a dimensionless parameter $\gamma$ given by

$$
\gamma=\frac{2}{8} k T_{c} q_{\max } / L_{1},
$$

where $k$ is Boltzmann's constant, $T_{c}$ is the nematic-isotropic transition temperature, $L_{1}$ is a material constant, and $q_{\max }$ is the cutoff wave vector for the continuum approximation description of nematic liquid crystals. $\gamma$ is related to $\theta_{0}$ by

$$
\gamma=\pi^{2}\left[A_{0}^{(2)}\right]_{N F}^{2}\left(T_{c} / T\right) \theta_{0}^{2},
$$

where $\left[A_{0}^{(2)}\right]_{M F}$ is the mean field value of $\left\langle P_{2}(\cos \theta)\right\rangle$ at absolute temperature $T$. Luckhurst ${ }^{21}$ has established an approximate theoretical prediction for $\theta_{0}$, which agrees with the EPR line shapes of some spin probes dissolved in nematic liquid crystals. No data are available for the materials used in our studies, but they should not differ greatly from the data on PAA ( $p-$ azoxyanisole). We therefore use Luckhurst's value of $\theta_{0} \approx 10^{\circ}$ at $T \approx T_{c}$ and $\left[A_{0}^{(2)}\right]_{M F}=0.429$ in Eq. (36) to obtain an estimate for $\gamma$ of

$$
\gamma \approx 0.055 \text {. }
$$

From Fig. 3 of Sheng's paper, it can be seen that a $\gamma$ of this order of magnitude introduces a shift in $\left[A_{0}^{(2)}\right]_{\text {measured }}$ and $\left[A_{0}^{(4)}\right]_{\text {measured }}$ toward smaller values by an amount small compared to our experimental uncertainty. These results are shown in Table I. We, therefore, conclude that our experiment is insensitive to director fluctuations provided $\theta_{0}$ for our materials is not unlike that for PAA.

(2) Noncylindrical molecules. Suppose one relaxes the assumption of cylindrical symmetric molecules. Then, the appropriate orientational distribution function might look like the one in Eq. (4) (for a molecule with two mutually perpendicular planes of symmetry). The principal molecular axes 1 and 2 will then have an anisotropic distribution around the 3 -axis (see Fig. 1) and, for $L=2$ and 4 , we will have nontrivial order parameters $A_{0}^{(2)}$ and $A_{0}^{(4)}$ characterizing the "axial" ordering with respect to the director, and $A_{2}^{(2)}, A_{2}^{(4)}$, and $A_{4}^{(4)}$ characterizing the "azimuthal" ordering around the major molecular axis.

It follows that, in addition to the parameters in Eq. (27), we must define quantities

$$
\begin{aligned}
& E=\left(1 / A^{2}\right)\left[\frac{1}{4}\left(\alpha_{11}^{\prime}-\alpha_{22}^{\prime}\right)^{2}-\alpha_{12}^{\prime 2}\right], \\
& F=\left(1 / A^{2}\right)\left(\alpha_{13}^{\prime 2}-\alpha_{23}^{\prime 2}\right), \\
& G=\left(1 / A^{2}\right)\left(\alpha_{11}^{\prime}-\alpha_{22}^{\prime}\right),
\end{aligned}
$$

where $A$ has been defined above, and the following terms

\begin{tabular}{|c|c|c|c|c|c|}
\hline \multirow{2}{*}{$\begin{array}{l}\text { Temperature } \\
\frac{T_{N T}-T}{T_{N}}\end{array}$} & \multirow[b]{2}{*}{$\begin{array}{l}T_{N I}-T \text { for } \\
T_{N I}=320^{\circ} \mathrm{K} \\
\left({ }^{\circ} \mathrm{C}\right)\end{array}$} & \multicolumn{2}{|c|}{$A_{0}^{(2)}$} & \multicolumn{2}{|c|}{$A_{0}^{(4)}$} \\
\hline & & $\begin{array}{l}\gamma=0 \\
(M-S)\end{array}$ & $\begin{array}{l}\gamma=0.055 \\
\left(\theta_{0}=10^{\circ}\right)\end{array}$ & $\begin{array}{l}\gamma=0 \\
(M-5)\end{array}$ & $\begin{array}{l}\gamma=0.055 \\
\left(\theta_{0}=10^{\circ}\right)\end{array}$ \\
\hline 0.092 & 29.34 & 0.615 & 0.602 & 0.256 & 0.239 \\
\hline 0.055 & 17.72 & 0.563 & 0.549 & 0.211 & 0.194 \\
\hline 0.0099 & 3.18 & 0.465 & 0.447 & 0.141 & 0.124 \\
\hline $8.63 \times 10^{-4}$ & 0.276 & 0.433 & 0.413 & 0.122 & 0.105 \\
\hline
\end{tabular}
have to be added to Eq. (27).

$$
\begin{aligned}
A^{-2}\left\langle\left(\alpha_{L}^{\prime}\right)_{x x}^{2}\right\rangle:- & \sqrt{\frac{2}{3}}\left(\frac{2}{7} F+\frac{1}{3} A G-\frac{2}{21} A D G\right) A_{2}^{(2)} \\
+ & \frac{3}{7} \sqrt{\frac{2}{5}}\left(F+\frac{1}{4} A D G\right) A_{2}^{(4)}+(3 E \sqrt{70}) A_{4}^{(4)}, \\
A^{-2}\left\langle\left(\alpha_{L}^{\prime}\right)_{x y}^{2}\right\rangle:= & \frac{1}{7} \sqrt{\frac{2}{3}}(3 F-A D G) A_{2}^{(2)}+\frac{1}{7} \sqrt{\frac{2}{5}}\left(F+\frac{1}{4} A D G\right) A_{2}^{(4)} \\
& +\left(E / \sqrt{70) A_{4}^{(4)}},\right. \\
A^{-2}\left\langle\left(\alpha_{L}^{\prime}\right)_{z z}^{2}\right\rangle:= & \sqrt{\frac{2}{3}}\left(\frac{2}{7} F+\frac{1}{3} A G-\frac{2}{21} A D G\right) A_{2}^{(2)} \\
& +\frac{2}{7} \sqrt{\frac{2}{5}}(4 F+A D G) A_{2}^{(4)}+(8 E / \sqrt{70}) A_{4}^{(4)} .
\end{aligned}
$$

TABLE I. Experimental values of the molecular field order parameters estimated by taking into account the broadening of the distribution of the director. 
TABLE II. Estimated corrections to the order parameters $A_{0}^{(2)}$ and $A_{0}^{(4)}$ calculated under the assumption of cylindrical molecular symmetry.

\begin{tabular}{l|ll|lllll}
\hline \hline & \multicolumn{5}{|c|}{$\begin{array}{c}\text { Results after } \\
\text { first order } \\
T_{N I}-T\end{array}$} & $\begin{array}{l}\text { Cylindrical } \\
\text { symmetry }\end{array}$ & \multicolumn{5}{c}{$\begin{array}{c}\text { corrections } \\
\left({ }^{\circ} \mathrm{C}\right)\end{array}$} & $A_{0}^{(2)}$ & $A_{0}^{(4)}$ & $A_{2}^{(2)}$ & $A_{2}^{(4)}$ & $A_{4}^{(4)}$ & $A_{0}^{(2)}$ & $A_{0}^{(4)}$ \\
\hline 30 & 0.657 & 0.151 & 0.030 & 0.075 & 0.000 & 0.668 & 0.210 \\
1.5 & 0.580 & 0.052 & 0.036 & 0.082 & 0.000 & 0.589 & 0.113 \\
4 & 0.444 & -0.106 & 0.048 & 0.104 & 0.000 & 0.436 & -0.044 \\
\hline
\end{tabular}

For the uniaxial cyano stretching vibration we have simply

$$
\begin{aligned}
& E=\left(1 / 4 A^{2}\right)(1-a)^{2} \sin ^{4} \beta_{0}=B, \\
& F=\left(1 / A^{2}\right)(1-a)^{2} \sin ^{2} \beta_{0} \cos ^{2} \beta_{0}=C, \\
& G=\left(1 / A^{2}\right)(1-a) \sin ^{2} \beta_{0} .
\end{aligned}
$$

We are not able to solve this problem exactly as there are not enough measurements to fix the three new unknown parameters. However, we can proceed in the following way to make some estimation of how serious these effects might be. We assume for simplicity that the statistics involving $\beta$ and $\gamma$ are independent so that the distribution of the molecules around their major axes deviates from isotropic as ${ }^{46}$

$f^{\prime}(\gamma)=\int_{0}^{\pi} \sin \beta d \beta \int_{0}^{2 r} d \alpha f(\alpha, \beta, \gamma) \doteq \frac{1}{2}(1+\delta \cos 2 \gamma)$,

which gives

$$
\begin{aligned}
& A_{2}^{(2)}=\frac{1}{2} \sqrt{\frac{3}{2}}\left\langle\sin ^{2} \beta \cos 2 \gamma\right\rangle \doteq \frac{1}{2} \sqrt{\frac{3}{2}}\left\langle\sin ^{2} \beta\right\rangle\langle\cos 2 \gamma\rangle \\
& =\frac{1}{4} \delta \sqrt{\frac{3}{2}}\left\langle\sin ^{2} \beta\right\rangle, \\
& A_{2}^{(4)}=\frac{1}{4} \sqrt{\frac{5}{2}}\left\langle\sin ^{2} \beta\left(7 \cos ^{2} \beta-1\right) \cos 2 \gamma\right\rangle \\
& \doteq \frac{1}{8} \delta \sqrt{\frac{5}{2}}\left\langle\sin ^{2} \beta\left(7 \cos ^{2} \beta-1\right)\right\rangle, \\
& A_{2}^{(4)}=\frac{1}{8} \cdot \sqrt{\frac{35}{2}}\left\langle\sin ^{4} \beta \cos 4 \gamma\right\rangle \doteq 0 .
\end{aligned}
$$

The effect of a nonzero $\delta$ is greater for $A_{2}^{(4)}$ than for $A_{2}^{(2)}$ according to Eq. (40). For conventional measurements of $A_{0}^{(2)}$, where only $A_{2}^{(2)}$ contributes, this has been discussed by Alben et al ${ }^{5}$ for PAA. The value of $\delta$ in that case was found to be 0.3 , independent of temperature. ${ }^{47}$ A positive value for 6 means that the planes of the molecules are on the average more likely to lie in the planes determined by the director and the major molecular axes. In other words, steric interactions can significantly influence the ordering in nematic liquid crystals. ${ }^{48}$

We have estimated first order corrections to $A_{0}^{(2)}$ and $A_{0}^{(4)}$ by substituting into $\mathrm{Eq}$. (40) zeroth order values of $\left\langle\sin ^{2} \beta\right\rangle$ and $\left\langle\sin ^{2} \beta\left(7 \cos ^{2} \beta-1\right)\right\rangle$ calculated from our data under the assumption of cylindrical molecular symmetry, and setting $\delta=0.3$. The results are listed in Table II. We have also estimated the effect on $A_{0}^{(2)}$ and $A_{0}^{(4)}$ of nonzero $A_{4}^{(4)}$ and see a noticeable change in the results, even when $A_{4}^{(4)}$ is made of the same order of magnitude as $A_{2}^{(2)}$. Although negative values of $A_{2}^{(2)}$, $A_{2}^{(4)}$, and $A_{4}^{(4)}$ and/or of $\delta$ could be tried, and the resulting changes in $A_{0}^{(2)}$ and $A_{0}^{(4)}$ would be in the opposite sense compared with those produced by positive values (Table II), the arguments necessary to justify such values involve reasoning we believe to be unphysical.

Although the effects of noncylindrical statistics do result in positive shifts in $A_{0}^{(4)}$ by as much as 0.05 , this is just about equal to the experimental uncertainty in $A_{0}^{(4)}$. We believe that although the assumption of cylindrical molecular symmetry may result in a small systematic error in $A_{0}^{(4)}$, the magnitude of this error is no larger than 0.05 . The errors in the values of $A_{0}^{(2)}$ are considerably smaller.

\section{Comparison with existing theories of nematic order}

The principal theoretical efforts to predict the temperature dependence of nematic order are the mean field theory of Maier and Saupe and the extended version of this by Luckhurst et al. Essential to both of these efforts is the temperature dependence of the specific volume. This can be extracted empirically from the data on indices of refraction ${ }^{49}$ since only values relative to the value at the transition are required. This appears to be justified when we compare (for neat MBBA) the dependence of $V^{*}=V_{n}(T) / V_{n}\left(T_{N I}\right)$ upon $T^{*}=T / T_{N I}$ from absolute density measurements ${ }^{50}$ with that extracted from the indices data, as shown in Fig. 13. In fact this dependence, $V^{*}$ vs $T^{*}$, for both neat MBBA and the $20 \%$ mixture, is nearly identical. Together with the nearly identical functional dependence of $\Delta T$ vs $T^{*}$ for these two materials, this implies that the theoretical predictions for both are nearly the same. ${ }^{51}$ We neglect in the following the correction due to volume discontinuities at the transition, as we know it is very small. ${ }^{52}$ Also in

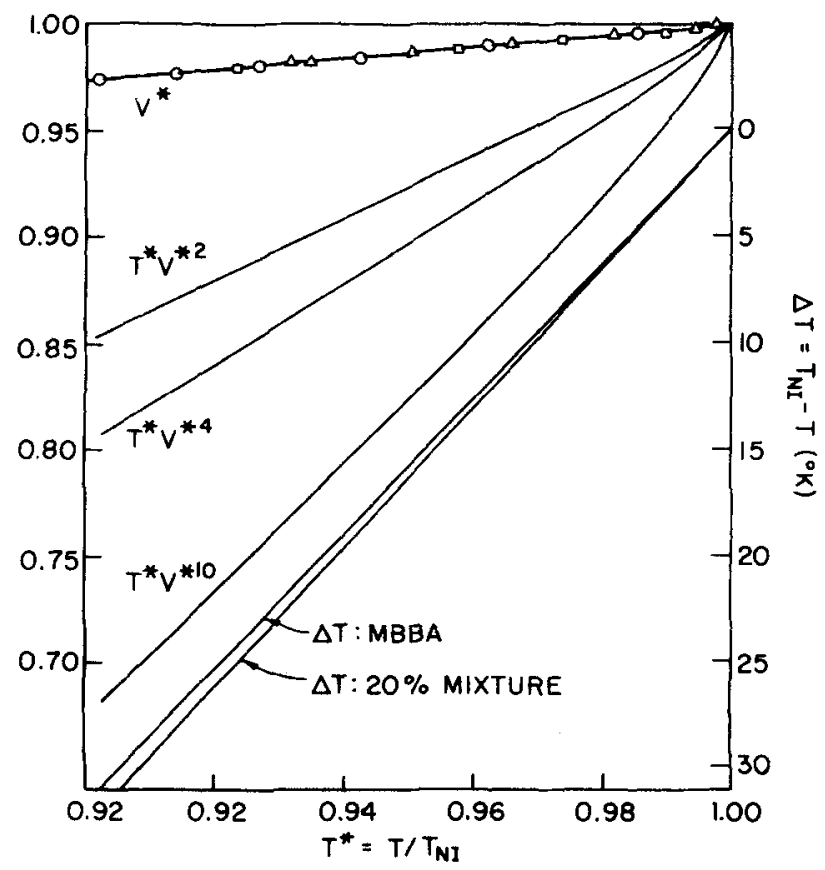

FIG. 13. Temperature dependence of $V^{*}=V_{n}(T) / V_{n}\left(T_{N I}\right)$ and $T^{*} V^{* m}$ with respect to $T^{*}=T / T_{N I}$. Data points are from absolute density measurements ( $\Delta$ from Ref. 50 ), relative values extracted from indices of refraction $[0: 20 \%$ mixture, $0:$ neat MBBA]. 
TABLE III. Predicted order parameters at the transition density by hard-rod models. The various models are referred to in Sec. II. Except for the Onsager's approximation, an aspect ratio of $L / D$ $=1.7$ is assumed. Numbers not available in the literature are marked by *.

\begin{tabular}{lll}
\hline \hline Model & $A_{0}^{(2)}$ & $A_{0}^{(4)}$ \\
\hline Onsager $^{\mathbf{2}}$ & 0.78 & 0.50 \\
Alben $^{\mathrm{b}}$ & 0.48 & $*$ \\
Lasher $^{\mathrm{c}}$ & 0.60 & $*$ \\
Cotter $^{\mathrm{d}}$ & 0.65 & $*$ \\
\hline \hline
\end{tabular}

${ }^{2}$ L. Onsager, Ann. N. Y. Acad. Sci. 51, 627 (1949).

bR. Alben, Mol. Cryst. Liq.

Cryst. 13, 193 (1971).

${ }^{\circ} \mathrm{G}$. Lasher, J. Chem. Phys, 53, 4141 (1970).

${ }^{d}$ M. A. Cotter, Phys. Rev. A 10, 625 (1974).

Fig. 13 we show the temperature dependence of $T^{*} V^{* m}$ for a number of integers $m$.

Following the work of Maier-Saupe and HumphriesJames-Luckhurst, specific computation schemes were developed that reproduced these earlier calculations of nematic order parameters, Details of these procedures are available in the $\mathrm{Ph} . \mathrm{D}$. thesis of one of us (SJ). ${ }^{41}$ The Maier-Saupe (MS) theory predicts at each $\alpha\left(T_{N I}\right)$ / $\alpha(T)=T^{*} V^{* 2}$ theoretical $A_{0}^{(2)}$ and $A_{0}^{(4)}$, and gives at the transition $\alpha\left(T_{N I}\right)=4.541, A_{0}^{(2)}=0.4289$, and $A_{0}^{(4)}=0.1198$. In the nematic phase at each $\Delta T$, for which $\alpha^{*}$ can be fixed from Fig. 13, we have thus a pair of calculated $A_{0}^{(2)}$ and $A_{0}^{(4)}$. These are also shown in Fig. 9 as the solid lines. Although they do show the qualitative behavior of the measured quantities $A_{0}^{(2)}$, there are significant quantitative discrepancies. The results of the Humphries-JamesLuckhurst (HJ L) theory are also shown in Fig. 9 as dashed lines. Here the parameters $\alpha\left(T_{N I}\right)=4.650$ and $\lambda=-0.55$ are chosen to fit the experimental $A_{0}^{(2)}=0.318$ at the transition, which implies a predicted value $A_{0}^{(4)}$ $=0.050$. With this pair of $\alpha\left(T_{N I}\right)$ and $\lambda$, successive values of $A_{0}^{(2)}$ and $A_{0}^{(4)}$ are calculated as functions of $\alpha^{*}$ $=\alpha(T) / \alpha\left(T_{N I}\right)$. The parameter $m=10 \pm 2$ is then determined by the best fit of the experimental $A_{0}^{(2)}$ with the calculated values at $\alpha^{*}=T^{*} V^{* m}$ in the nematic phase with the aid of Fig. 13. Although with extra parameters in the HJL theory the fit for $A_{0}^{(2)}$ is perfect, the predictions for $A_{0}^{(4)}$ are not significantly improved. Also, the value of $\lambda=-0.55$ seems to indicate a poor convergence of the pseudopotential expansion, if this expansion is adequate. Furthermore, the choice of $m=10 \pm 2$ that is required to fit the $A_{0}^{(2)}$ data implies an unusually strong dependence of the order parameters on density. With more reasonable values of $m$ (for example, $m=4$ ), the fit of the HJL theory, although not perfect for $A_{0}^{(2)}$, improves upon the MS theory. The predictions for $A_{0}^{(4)}$, however, still disagree significantly with the data. Even if the estimated spreading of the director is taken into account, these discrepancies prevail. We list in Table III the order parameters predicted by various hard-rod models at the transition density, which may also be compared with the experimental data.

It would also be interesting to compare the data with the phenomenological theories using different fitting schemes. For example, since the mean field theories do not necessarily include the long wavelength director fluctuations, one might expect that some of the predictions might be improved with the fitting being made far away from the transition. However, in this type of mean field theory, the ratio of $A_{0}^{(4)} / A_{0}^{(2)}$ is basically fixed and will be seen to disagree with our results as we present further experimental facts.

\section{E. Examination of the truncated orientational distribution function}

Since it is clear that the Raman studies just described obtain order parameters $A_{0}^{(4)}$ that disagree with theoretical predictions, it is desirable to interpret the significance of this disagreement. Consider the quantity $\sigma$ defined by

$$
\sigma=\left(\cos ^{2} \beta-\left\langle\cos ^{2} \beta\right\rangle\right) /\left\langle\cos ^{2} \beta\right\rangle .
$$

By Schwartz's inequality we have

$$
\left\langle\sigma^{2}\right\rangle=\left(\left\langle\cos ^{4} \beta\right\rangle-\left\langle\cos ^{2} \beta\right\rangle^{2}\right) /\left\langle\cos ^{2} \beta\right\rangle^{2} \geq 0 .
$$

Note that in the isotropic phase $\left\langle\sigma^{2}\right\rangle=0.8$ and in the perfectly aligned state $\left\langle\sigma^{2}\right\rangle=0$. However, $\left\langle\sigma^{2}\right\rangle=0$ does not imply perfect alignment. Rather, a state in which the principal axis of every molecule makes some definite angle $\beta$ or $\pi-\beta$ with respect to the $z$ axis will have $\left\langle\sigma^{2}\right\rangle=0$, even if all azimuthal angles are equally probable. Taking

$$
\begin{aligned}
& A_{0}^{(2)}=\frac{1}{2}\left\langle 3 \cos ^{2} \beta-1\right\rangle, \\
& A_{0}^{(4)}=\frac{1}{8}\left\langle 35 \cos ^{4} \beta-30 \cos ^{2} \beta+3\right\rangle,
\end{aligned}
$$

Eq. (42) can be written as

$$
\begin{aligned}
A_{0}^{(4)}=\frac{1}{72}[140(1 & \left.+\left\langle\sigma^{2}\right\rangle\right)\left(A_{0}^{(2)}\right)^{2}-20\left(2-7\left\langle\sigma^{2}\right\rangle\right) A_{0}^{(2)} \\
& \left.-7\left(4-5\left\langle\sigma^{2}\right\rangle\right)\right] .
\end{aligned}
$$

Figure 14 is a plot of $A_{0}^{(4)}$ vs $A_{0}^{(2)}$ obtained from Eq. (43) by assuming several fixed values of $\left\langle\sigma^{2}\right\rangle$. The experimental points, as well as those predicted by the mean field theories, are shown. From this plot one can read off the experimental values of $\left\langle\sigma^{2}\right\rangle$ and see that they satisfy both the Schwartz inequality and the expectation that $\left\langle\sigma^{2}\right\rangle_{\text {nom }}$ is less than $\left\langle\sigma^{2}\right\rangle_{\text {iso }}=0.8$. One can see further that the increase in the nematic order accompanying a decrease in temperature also decreases the empirical value of $\left\langle\sigma^{2}\right\rangle$. Viewed this way, there is nothing unphysical about negative values of $A_{0}^{(4)}$.

A slightly different way of viewing this same result is to consider the first three terms in the orientational distribution function, Eq. (6),

$$
f_{\overline{\mathfrak{u}}}^{\mathrm{III}}(\beta)=\frac{1}{2}\left[1+5 A_{0}^{(2)} P_{2}(\cos \beta)+9 A_{0}^{(4)} P_{4}(\cos \beta)\right] .
$$

In Fig. 15 we plot $f_{\frac{1}{u}}^{\mathrm{III}}(\beta)$ for two temperatures using our experimental values of $A_{0}^{(2)}$ and $A_{0}^{(4)}$ and values calcu- 


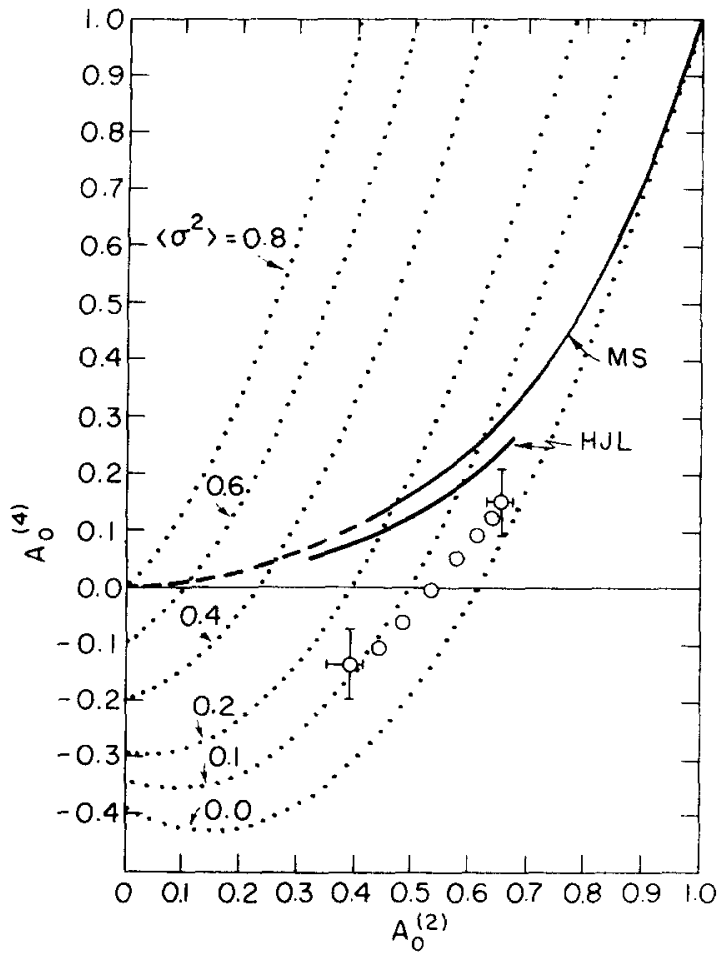

FIG. 14. Variation of $A_{0}^{(4)}$ vs $A_{0}^{(2)}$. Circles are data points from Raman measurements. Dotted lines are contours of constant $\left\langle\sigma^{2}\right\rangle$. Solid lines are predictions of mean field theories.

lated from the HJL theory after the parameters are adjusted to obtain good agreement with $A_{0}^{(2)}$. Note that although $f_{\hat{u}}(\beta)$ is positive definite $f_{\hat{u}}^{\mathrm{III}}(\beta)$ need not be. We also show in the same figure $f_{\hat{u}}^{\mathrm{II}}(\beta)$ for values estimated from Table $I$. The principal results to see in this figure is that the $\mathrm{BBCA}$ molecules (and presumably also the MBBA hosts in the mixture) have a stronger tendency to be tipped away from the nematic axis than pre- dicted by the mean field theory. This tendency is strongest near the transition. We mention here two possibilities for this disagreement. First, the assumption of a rigid molecule may be unrealistic. To the extent that molecules are nonrigid, there is no unique choice of a single set of order parameters, as we have mentioned in Sec. I. In the present case, the agreement between the NMR results and all the other measurements of $A_{0}^{(2)}$ suggests that the $A_{0}^{(2)}$ may be suitable if the angle $\beta$ is defined by the rigid part of the molecules, that is, the central core, with respect to the director. In this case, there is no a priori reason to believe that the mean field theory should apply only to one part of a complex molecule. For example, if an $L-$ shaped isomer is relatively probable, the observed behavior of $A_{0}^{(4)}$ may be rationalized. Second, it is still possible that the rigidity of the molecules is approximately acceptable but the mean field theory fails because it neglects the interactions that induce splay on a microscopic scale, that is, pair correlations ${ }^{53.54}$ in which neighboring molecular axes are not parallel. For example, the dipole-dipole interactions could do this.

To summarize, although the existing statistical theories of nematic ordering are qualitatively useful for the understanding of the gross leatures, they are not quantitatively reliable.

\section{STUDY OF THE OTHER VIBRATIONAL BANDS}

\section{A. Introduction}

In this section we will describe our attempts to use the intrinsic Raman spectra of the neat liquid crystal to obtain the same information that was previously obtained from the probe molecule and its uniaxial cyanovibration. Most liquid crystals are not made from molecules with Raman active modes that have the same simple symmetry and orientation as the cyano group.
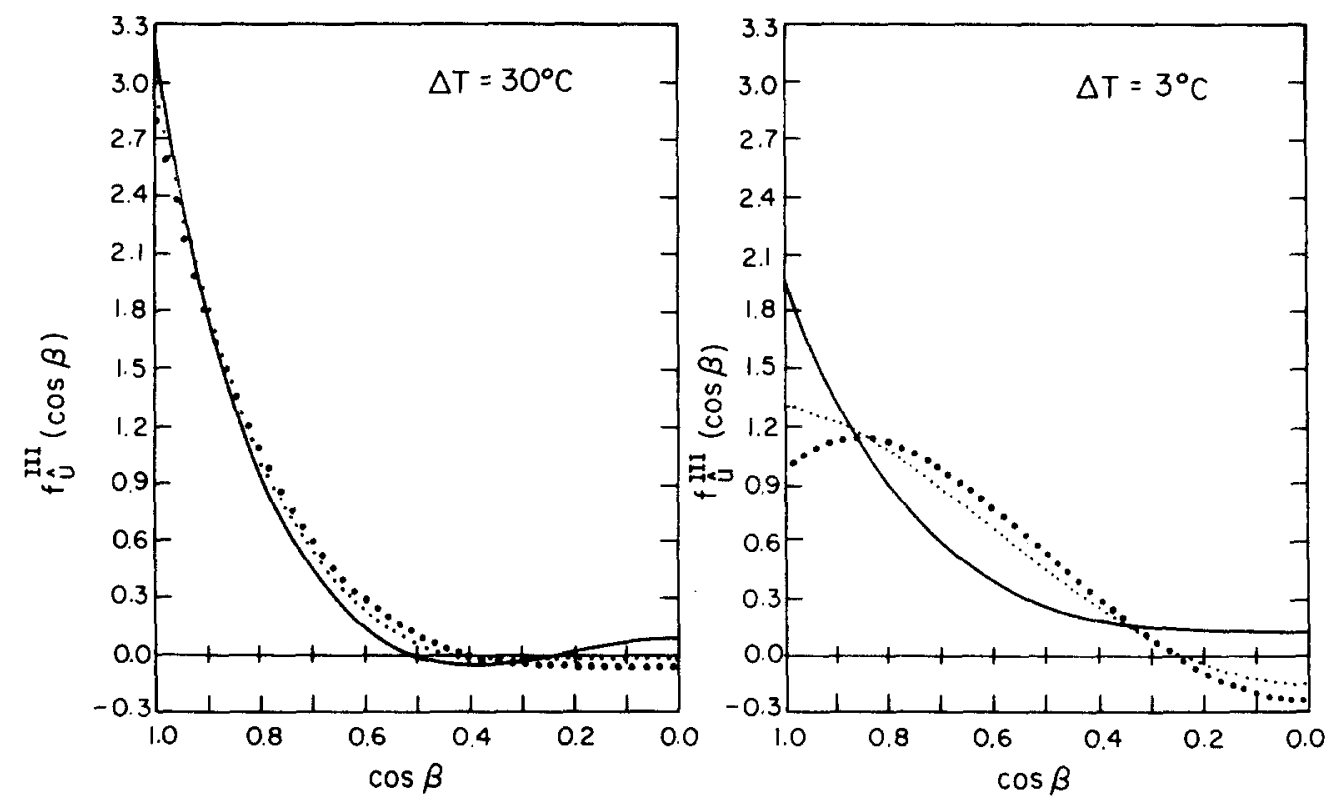

FIG. 15. Plots of the theoretical and experimental truncated angular distribution function $f_{\hat{u}}^{I I}(\beta)$ : solid lines, HJL theory; large dots, Raman measurements from Fig. 13; small dots, estimation from Table I. 


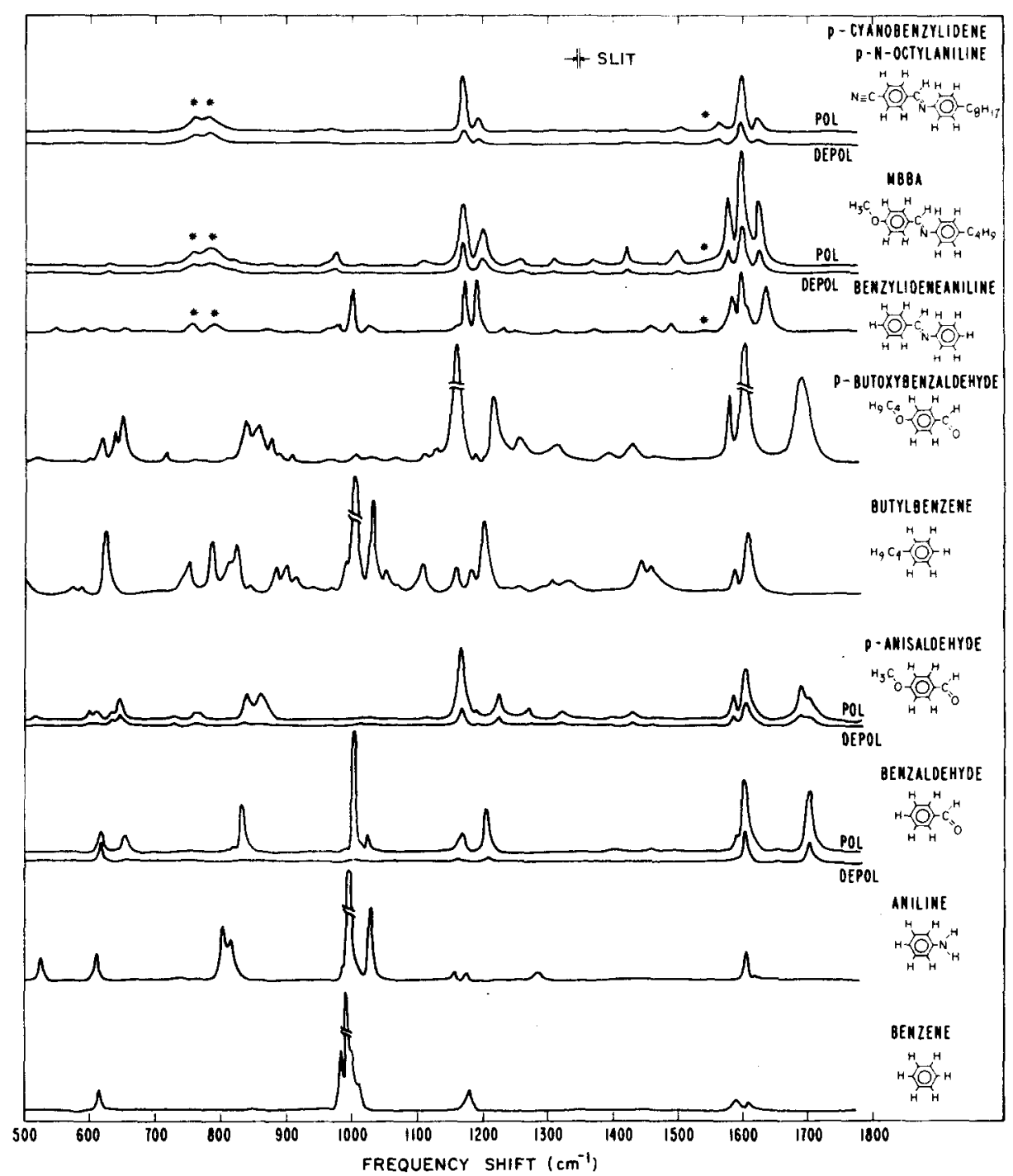

FIG. 16. Raman spectra of the series of compounds used in the identification scheme. The top three are taken from isotropic solutions in solvent $\mathrm{CCl}_{4}$ whose spectral lines are marked by *. There is no correlation among the vertical scales for each compound.
Nevertheless, on comparing the Raman spectra of a series of increasingly complex molecules as shown in Fig. 16, one observes that in going from benzene to the larger molecules there appear both new Raman modes associated with the different groups attached to the phenyl rings, and other bands which seem to be associated with the rings themselves. However, after the relatively rigid and heavy central section of the Schiff's base is built up, the addition of the tails merely results in new bands of weak vibrations associated with the alkoxy or alkyl end groups. The main structure of those bands associated with vibrations in the central core is not perturbed significantly by vibrations in the tails. We have also taken similar Raman spectra of isotropic solutions of the $20 \%$ mixture, CBOOA and 40.8 , and they all appear to be very close, if not identical, to those of the MBBA and $p$-cyanobenzylidene- $p-N$-octylaniline.

The criteria of selecting useful bands are threefold: first, they should be associated with the rigid central part of the liquid crystal molecule so that an unambiguous comparison with the various other data is possible; second, there should be enough signal to make the intensity measurements feasible; third, we need to have bands whose Raman intensities are sensitive to the molecular orientation. Apparently, from Fig. 16 there are five bands at approximately ${ }^{55} 1170,1200,1576$, 1597 , and $1625 \mathrm{~cm}^{-1}$ which appear to be associated with vibrations in the central core of the Schiff's base liquid crystal molecules. Furthermore, the measured depolarization ratios of these bands do show very characteristic changes with respect to the orientational order. These data will be presented below. Some preliminary spectra have also been taken in the region $2700-3800$ $\mathrm{cm}^{-1}$, where the bands are associated with the proton vibrations, which may be useful in the studying of the statistics of the tails.

\section{B. Data from nematic samples}

The Raman depolarization ratios $R_{1}, R_{2}$, and $R_{3}$ of the 1576,1597 , and $1625 \mathrm{~cm}^{-1}$ bands have been measured as functions of both temperature and thickness for the $20 \%$ mixture and neat MBBA. We plot in Fig. 17 typical data of the $1597 \mathrm{~cm}^{-1}$ band for the $20 \%$ mixture at two thicknesses. The temperature dependence of $R_{1}$ and $R_{2}$ is very similar to that shown in Fig. 6. The variation of $R_{3}$ is less dramatic. $R_{1 \text { so }}$ is also shown in the same plot, and there is no observable dependence on either 


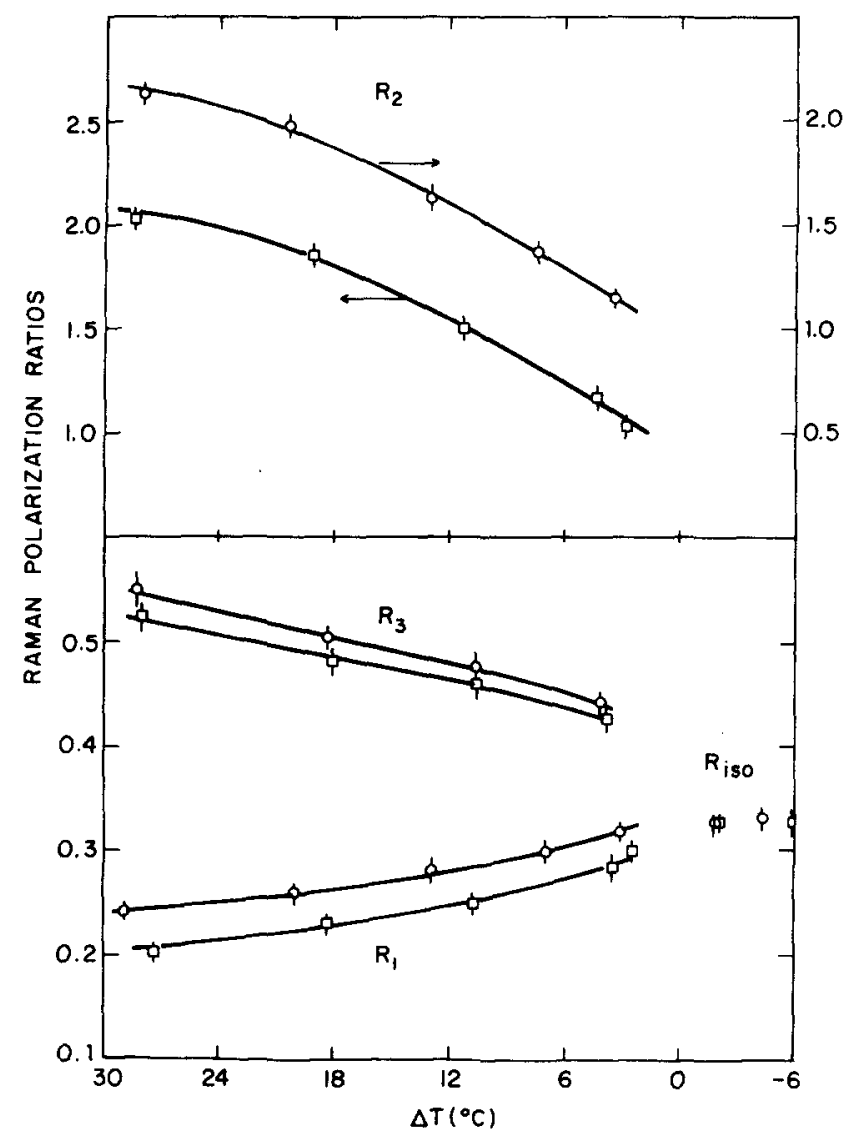

FIG. 17. Raman depolarization ratios $R_{1}, R_{2}, R_{3}$, and $R_{\text {1so }}$ of the $1597 \mathrm{~cm}^{-1}$ band for samples of $20 \% \mathrm{BBCA}$ in MBBA. Circles: $76.1 \mu$; squares: $51.5 \mu$ thick.

thickness or temperature within a range of roughly $7{ }^{\circ} \mathrm{C}$ above the transition. The data on the other bands, as well as the data for MBBA and the measurements for the $10 \%$ mixture at one temperature, are tabulated in Tables IV-VI.

\section{Discussion}

It is far from obvious that the equations derived above and applied to the cyano-vibration will be applicable here. For one thing, the cyano-vibration is highly localized in the molecule and its frequency is well separated from the other vibronic modes. Intermolecular coupling effects could be much more important for the

TABLE IV. Raman depolarization ratios of vibrational bands at 1576 and $1625 \mathrm{~cm}^{-1}$ for $20 \% \mathrm{BBCA}$ in MBBA. Ratios other than $R_{\text {iso }}$ are extrapolated values at zero thickness.

\begin{tabular}{lllll}
\hline $\begin{array}{l}\Delta \omega \\
\left(\mathrm{cm}^{-1}\right)\end{array}$ & $\begin{array}{l}\Delta T \\
\left({ }^{\circ} \mathrm{C}\right)\end{array}$ & \multicolumn{1}{c}{$R_{1}$} & \multicolumn{1}{c}{$R_{2}$} & \multicolumn{1}{c}{$R_{3}$} \\
\hline 1576 & 25 & $0.160 \pm 0.010$ & $1.916 \pm 0.110$ & $0.496 \pm 0.020$ \\
& 15 & 0.182 & 1.530 & 0.464 \\
& 4 & $0.227 \pm 0.015$ & $1.105 \pm 0.060$ & $0.419 \pm 0.020$ \\
& $R_{150}=$ & $0.318 \pm 0.012$ & & \\
1625 & 25 & $0.145 \pm 0.011$ & $1.320 \pm 0.050$ & $0.362 \pm 0.020$ \\
& 15 & 0.165 & 1.145 & 0.353 \\
& 4 & $0.205 \pm 0.015$ & $0.892 \pm 0.070$ & $0.334 \pm 0.020$ \\
& $R_{150}=$ & $0.281 \pm 0.010$ & & \\
\hline
\end{tabular}

TABLE V. Raman depolarization ratios of vibrational bands at 1576,1597 , and $1625 \mathrm{~cm}^{-1}$ for neat MBBA. Ratios other than $R_{\mathrm{tso}}$ are extrapolated values at zero thickness.

\begin{tabular}{lllll}
\hline $\begin{array}{l}\Delta \omega \\
\left(\mathrm{cm}^{-1}\right)\end{array}$ & $\begin{array}{l}\Delta T \\
\left({ }^{\circ} \mathrm{C}\right)\end{array}$ & \multicolumn{1}{c}{$R_{1}$} & \multicolumn{1}{c}{$R_{2}$} & \multicolumn{1}{c}{$R_{3}$} \\
\hline 1576 & 16 & $0.157 \pm 0.010$ & $1.395 \pm 0.040$ & $0.481 \pm 0.030$ \\
& 10 & 0.179 & 1.246 & 0.451 \\
& 4 & $0.208 \pm 0.030$ & $1.094 \pm 0.080$ & $0.429 \pm 0.030$ \\
& $R_{\text {1so }}=$ & $0.343 \pm 0.015$ & & \\
1597 & 16 & $0.177 \pm 0.010$ & $1.370 \pm 0.070$ & $0.464 \pm 0.020$ \\
& 10 & 0.201 & 1.220 & 0.460 \\
& 4 & $0.228 \pm 0.020$ & $1.075 \pm 0.090$ & $0.457 \pm 0.015$ \\
& $R_{\text {iso }}=$ & $0.373 \pm 0.010$ & & \\
1625 & 16 & $0.148 \pm 0.010$ & $1.148 \pm 0.100$ & $0.370 \pm 0.020$ \\
& 10 & 0.170 & 1.077 & 0.363 \\
& 4 & $0.199 \pm 0.020$ & $0.994 \pm 0.050$ & $0.358 \pm 0.014$ \\
& $R_{\text {1so }}=$ & $0.310 \pm 0.010$ & & \\
\hline \hline
\end{tabular}

modes being considered here and the temperature dependence of the Raman intensities could have contributions in addition to that due to the macroscopic nematic order parameters. Experimentally, there is also the question as to whether each of these bands is from a single vibration. In fact, it is quite possible that some of the bands are of composite profiles. We will, however, assume for simplicity that they all can be represented by a simple real phenomenological polarizability tensor and proceed on the basis of the assumptions made earlier. Eventually we will evaluate the results thus obtained and judge empirically whether or not the assumptions are valid.

We consider first the data for the $20 \%$ mixture. The polarizability tensor in the local frame has the general biaxial form given by Eq. (25). We first solve from the measured ratios $R_{1}, R_{2}, R_{3}$, and $R_{\text {iso }}$ for the four unknowns $A_{0}^{(2)}, A_{0}^{(4)}, a$, and $b$ by Eqs. (27), (28), and (33) with $\beta_{0}$ being an adjustable parameter. This is essentially to assume cylindrical molecular symmetry and ignore the angle $\alpha_{0}$ as we have mentioned at the end of Sec. III. By comparing the calculated values $A_{0}^{(2)}$ with those shown in Fig. 9, we obtain an angle $\beta_{0}$ for each of these bands. This angle is determined to be between $0^{\circ}$ and $2^{\circ}$ for the 1576 and the $1625 \mathrm{~cm}^{-1}$ bands and $4^{\circ}$ and $6^{\circ}$ for the $1597 \mathrm{~cm}^{-1}$ band. Taking these values for $\beta_{0}$, the values of $A_{0}^{(4)}$ calculated here are all seem to match reasonably well with those shown in Fìg. 9. The parameter $\alpha_{0}$ has as yet been left uncommitted. We

TABLE VI. Raman depolarization ratios of vibrational bands at 1576, 1597, and $1625 \mathrm{~cm}^{-1}$ for $10 \% \mathrm{BBCA}$ in MBBA. Ratios other than $R_{\text {iso }}$ are extrapolared values at zero thickness.

\begin{tabular}{llccc}
\hline \hline $\begin{array}{l}\Delta \omega \\
\left(\mathrm{cm}^{-1}\right)\end{array}$ & $\begin{array}{l}\Delta T \\
\left({ }^{\circ} \mathrm{C}\right)\end{array}$ & $R_{1}$ & $R_{2}$ & $R_{3}$ \\
\hline 1576 & 6.4 & $0.191 \pm 0.006$ & $1.058 \pm 0.040$ & $0.441 \pm 0.017$ \\
& $R_{1 \mathrm{so}}=$ & $0.341 \pm 0.010$ & & \\
1597 & 6.4 & $0.212 \pm 0.012$ & $1.140 \pm 0.080$ & $0.471 \pm 0.015$ \\
& $R_{\text {iso }}=$ & $0.370 \pm 0.012$ & & \\
1625 & 6.4 & $0.183 \pm 0.005$ & $0.882 \pm 0.035$ & $0.419 \pm 0.018$ \\
& $R_{\text {iso }}=$ & $0.321 \pm 0.010$ & & \\
\hline
\end{tabular}


thus try different values for $\alpha_{0}$ with those angles $\beta_{0}$ obtained above using, instead, the general relation for $\alpha_{i j}$ in Appendix I and Eqs. (27) and (28). The reiterated values for both $A_{0}^{(2)}$ and $A_{0}^{(4)}$ are not appreciably different from those obtained by setting $\alpha=0$. This is mainly because $\beta_{0}$ is very close to zero and presumably the vibrations are such that the tensor element of the Raman polarizability in the local frame $\left(\alpha_{d}^{\prime}\right)_{33}$ is very different from either $\left(\alpha_{d}^{\prime}\right)_{11}$ or $\left(\alpha_{d}^{\prime}\right)_{22}$ in magnitude. Since the polarizability is in general biaxial, from the analysis above, we have two sets of solutions for $a$ and $b$ (see Appendix III for the procedure used to obtain numerical solutions). The reason is readily understood from the form of the equations in Appendix I and Sec. III. When $\beta_{0}=0$, the interchange of $a$ and $b$ essentially interchanges the axes 1 and 2 in the local frame. Therefore, exactly the same order parameters $A_{0}^{(2)}$ and $A_{0}^{(4)}$ are obtained from either $\left(a_{1}, b_{1}\right)$ or $\left(a_{2}, b_{2}\right)=\left(b_{1}, a_{1}\right)$. This is simply a consequence of the assumption of cylindrical molecular symmetry. These are shown in Table VI for the 1576 and $1625 \mathrm{~cm}^{-1}$ bands. We see in the same table when $\beta_{0} \neq 0$, that is, for the $1597 \mathrm{~cm}^{-1}$ band, there are again two sets of $a$ and $b$ although the values are no longer directly interchangeable, as slight differences exist between $a_{1}$ and $b_{2}$ as well as between $a_{2}$ and $b_{1}$. However, if we rotate the axes 1 and 2 by $90^{\circ}$ and interchange $a$ and $b$ afterwards, we get once again the other pair, that is, $\left(a_{1}, b_{1}\right)_{\alpha_{0}=0^{\circ}}=\left(b_{2}, a_{2}\right)_{\alpha_{0}=90^{\circ}}$ and $\left(a_{2}, b_{2}\right)_{\alpha_{0}=0^{\circ}}$ $=\left(b_{1}, a_{1}\right)_{\alpha_{0}=90^{\circ}}$. Not knowing $\alpha_{0}$, we are not certain which set to choose. Nevertheless, these two sets of $(a, b)$ yield order parameters $A_{0}^{(2)}$ and $A_{0}^{(4)}$ which are only slightly different.

Before drawing any conclusion, however, we can apply a further test to see if the calculated values of $a$ and $b$ are consistent with our assumptions. The essence of the Raman technique is to extract the order parameter from the Raman intensities, which are proportional to $\left\langle\left[\alpha_{L}^{\prime}(\Omega)\right]^{2}\right\rangle$ with

$$
\alpha_{L}^{\prime}(\Omega)=\left(\alpha_{L}^{\prime}\right)_{t}+\left[\alpha_{L}^{\prime}(\Omega)\right]_{a},
$$

where $\left(\alpha_{t}^{t}\right)_{t}$ is the isotropic part of the polarizability tensor $\alpha_{L}^{\prime}(\Omega)$,

$$
\left(\alpha_{L}^{\prime}\right)_{t}=\frac{1}{3} I \operatorname{tr}\left[\alpha_{L}^{\prime}(\Omega)\right]=\frac{1}{3} I \operatorname{tr}\left(\alpha_{d}^{\prime}\right)=\left(\alpha_{d}^{\prime}\right)_{t},
$$

with $\alpha_{a}^{\prime}$ being in the local frame, Eq. (25), and being temperature independent. $\left[\alpha_{L}^{\prime}(\Omega)\right]_{a}$ is the anisotropic part of $\alpha_{L}^{\prime}(\Omega)$,

$$
\left[\alpha_{L}^{\prime}(\Omega)\right]_{a}=\mathbf{R}(\Omega)\left(\alpha_{a}^{\prime}\right)_{a} R^{+}(\Omega) .
$$

The subscripts $t$ and $a$ are referred to respectively the trace and the anisotropy. Therefore, a good test for the consistency of this technique is to compare the calculated values for trace and the anisotropy of the polarizability $\alpha^{\prime}$ in the local frame at various temperatures.

$$
\begin{aligned}
& {\left[\left(\alpha_{d}^{\prime}\right)_{t}\right]_{i j}=\frac{1}{3} \delta_{i j} \operatorname{tr}\left(\alpha_{d}^{\prime}\right),} \\
& {\left[\left(\alpha_{d}^{\prime}\right)_{a}\right]_{i j}=\left(\alpha_{d}^{\prime}\right)_{i j}-\left[\left(\alpha_{d}^{\prime}\right)_{t}\right]_{i j} .}
\end{aligned}
$$

We believe the most significant way to make these comparisons is in terms of the following linear combinations:

$$
\begin{aligned}
& {\left[\left(\alpha_{d}^{\prime}\right)_{t}\right]_{11}=\left[\left(\boldsymbol{\alpha}_{d}^{\prime}\right)_{t}\right]_{22}=\left[\left(\boldsymbol{\alpha}_{d}^{\prime}\right)_{t}\right]_{33}=\frac{1}{3}(a+b+1),} \\
& \frac{\left[\left(\alpha_{d}^{\prime}\right)_{a}\right]_{11}}{\frac{1}{3} \operatorname{Tr}\left(\boldsymbol{\alpha}_{d}^{\prime}\right)}=\frac{2 a-b-1}{a+b+1}, \\
& \frac{\left[\left(\alpha_{d}^{\prime}\right)_{a}\right]_{22}}{\frac{1}{3} \operatorname{Tr}\left(\boldsymbol{\alpha}_{d}\right)}=\frac{2 b-a-1}{a+b+1}, \\
& \frac{\left[\left(\boldsymbol{\alpha}_{d}^{\prime}\right)_{a}\right]_{33}}{\frac{1}{3} \operatorname{Tr}\left(\alpha_{d}\right)}=\frac{2-a-b}{a+b+1},
\end{aligned}
$$

since the trace and the anisotropy of $\alpha_{d}^{\prime}$ in the local frame are then tested separately. These quantities deduced from the data of the $20 \%$ mixture are also included as the last four columns of Table VII. From this table, the near constancy of the quantities associated with the calculated $\alpha_{d}^{\prime}$, as well as the very good agreement for $A_{0}^{(2)}$ and $A_{0}^{(4)}$ here with those shown in Fig. 9, seems to support the integrity of the Raman technique.

The same analysis has also been carried out for the neat MBBA, and the results are listed in Table VIII. We notice that although $A_{0}^{(2)}$ agrees with the results for the $20 \%$ mixture, the $A_{0}^{(4)}, a$, and $b$ are quite different from those shown in Fig. 9 and Table VIII. The values $a$ and $b$ are, however, still self-consistent among themselves. Rather than concluding the technique has failed, we believe that this discrepancy simply indicates that the two liquid crystals, the $20 \%$ mixture, and the neat MBBA have different values for the order parameter $A_{0}^{(4)}$ even though they have the same $A_{0}^{(2)}$ at the same reduced temperature. The difference between the two liquid crystals can also be seen from the observed $R_{\text {ieo }}$ for the same bands. This simply means that the local modification of the polarizability is different for the two materials. The results for the $10 \%$ mixture at $\Delta T$ $=6.4{ }^{\circ} \mathrm{C}$ turn out to be very close to those of the neat MBBA. In Fig. 18 we plot the order parameters calculated in this section together with a reproduction of Fig. 9.

We conclude this section with the following remarks. First, if we estimate the effects of director fluctuations in the manner discussed earlier, the data are not explained by the mean field theories for neat liquid crystals like MBBA. Second, the difference in the order parameter $A_{0}^{(4)}$ between neat MBBA and the $20 \%$ mixture may very well be an indication of the different pair correlations in these two liquid crystals, since it is well known that the two types of molecules, MBBA and BBCA, have very different dc dielectric properties as indicated by the signs of the dielectric anisotropy. The fact that the results for the $10 \%$ mixture are very similar to those for neat MBBA can tentatively be rationalized by making the reasonable assumption that intermolecular interactions are smaller at this concentration. This, however, requires further experimental study.

\section{STUDY OF THE LIQUID CRYSTAL COMPOUND 40.8}

\section{A. Data}

The index of refraction data for the compound 40.8 are shown in Fig. 19 at two wavelengths, 5145 and 
TABLE VII. Calculated values of $A_{0}^{(2)}, A_{0}^{(4)}, a, b$, the trace, and the anisotropy for the three bands of the 20\% mixture. Uncertainties include all the statistical uncertainties listed in Table IV and $\beta_{0}=1^{\circ} \pm 1^{\circ}$ for the 1576 and $1625 \mathrm{~cm}^{-1}$ bands, $5^{\circ} \pm 2^{\circ}$ for the $1597 \mathrm{~cm}^{-1}$ band.

\begin{tabular}{|c|c|c|c|c|c|c|c|c|c|c|}
\hline \multirow[b]{2}{*}{$\Delta T\left({ }^{\circ} \mathrm{C}\right)$} & \multicolumn{7}{|c|}{$(a, b)$} & \multirow[b]{2}{*}{$\frac{2 a-b-1}{a+b+1}$} & \multirow[b]{2}{*}{$\frac{2 b-a-1}{a+b+1}$} & \multirow[b]{2}{*}{$\frac{2-a-b}{a+b+1}$} \\
\hline & $A_{0}^{(2)}$ & $A^{(4)}$ & (1) & & (2) & & $a+b+1$ & & & \\
\hline \multicolumn{11}{|c|}{$\Delta w=1576 \mathrm{~cm}^{-1}$} \\
\hline 25 & $0.650 \pm 0.017$ & $0.109 \pm 0.038$ & $-0.140=0.025$ & $0.198 \pm 0.134$ & & & $1.058 \mp 0.042$ & $-1.397 \pm 0.080$ & $-0.439 \pm 0.080$ & $1.836+0.115$ \\
\hline & & & & & 0.198 & -0.140 & 1.058 & -0.439 & -1.397 & 1.836 \\
\hline 15 & 0.591 & 0.039 & -0.153 & 0.216 & & & 1.063 & -1.432 & -0.390 & 1.822 \\
\hline \multirow{3}{*}{4} & & & & & 0.216 & -0.153 & 1.063 & -0.390 & -1.432 & 1.822 \\
\hline & $0.465 \pm 0.020$ & $-0.083 \pm 0.038$ & $-0.162 \pm 0.022$ & $0.235 \pm 0.047$ & & & $1.073+0.052$ & $-1.453 \pm 0.075$ & $-0.343 \pm 0.105$ & $1.796 \pm 0.140$ \\
\hline & & & & & 0.235 & -0.162 & 1.073 & -0.343 & -1.453 & 1. 769 \\
\hline \multicolumn{11}{|c|}{$\Delta \omega=1597 \mathrm{~cm}^{-1}$} \\
\hline 25 & $0.643 \pm 0.015$ & $.0 .151 \pm 0.039$ & $-0.123=0.028$ & $0.166=0.030$ & & & $1.043 \pm 0.041$ & $-1.354 \pm 0.090$ & $-0.523 \pm 0.075$ & $1.876 \pm 0.115$ \\
\hline & $0.647 \pm 0.015$ & $0.154 \pm 0.039$ & & & 0.173 & -0.130 & $1.042 \pm 0.041$ & $-0.502 \pm 0.075$ & $-1.374 \pm 0.090$ & $1.876 \pm 0.115$ \\
\hline 15 & 0.585 & 0.049 & -0.131 & 0.173 & & & 1.042 & -1.377 & -0.502 & 1.879 \\
\hline & 0.589 & .0 .049 & & & 0.179 & -0.137 & 1.042 & -0.485 & -1.394 & 1.879 \\
\hline 4 & $0.430 \pm 0.021$ & $-0.0 .85 \pm 0.037$ & $-0.1 .40 \neq 0.029$ & $0.180 \pm 0.047$ & & & $1.040 \pm 0.055$ & $-1.404 \pm 0.100$ & $-0.481 \pm 0.1 .15$ & $1.885 \pm 0.160$ \\
\hline & $0.433 \pm 0.021$ & $-0.087 \pm 0.037$ & & & 0.188 & -0.145 & $1.043 \pm 0.055$ & $-0.459 \pm 0.115$ & $-1.4 .17 \pm 0.100$ & $1.876=0.140$ \\
\hline \multicolumn{11}{|c|}{$\Delta \omega=1625 \mathrm{~cm}^{-1}$} \\
\hline 25 & $0.638 \pm 0.016$ & $0.114=0.038$ & $-0.097 \pm 0.025$ & $0.225 \pm 0.034$ & & & $1.128 \pm 0.042$ & $-1.258 \pm 0.042$ & $-0.402 \pm 0.075$ & $1.660 \pm 0.100$ \\
\hline & & & & & 0.225 & -0.097 & 1.128 & -0.402 & -1.258 & 1.660 \\
\hline 1.5 & 0.582 & 0.042 & -0.106 & 0.240 & & & 1.134 & -1.280 & $-0,365$ & 1.645 \\
\hline \multirow{3}{*}{4} & & & & & 0.240 & -0.106 & 1.134 & -0.365 & -1.280 & 1.645 \\
\hline & $0.456 \pm 0.020$ & $-0.079 \pm 0.040$ & $-0.114 \pm 0.030$ & $0.254 \pm 0.045$ & & & $1.140 \pm 0.054$ & $-1.300 \pm 0.093$ & $-0.332 \pm 0.130$ & $1.632 \pm 0.040$ \\
\hline & & & & & 0.254 & -0.114 & 1.140 & -0.332 & -1.300 & 1.632 \\
\hline
\end{tabular}

$6328 \AA$. It is interesting to notice the nearly continuous variation between the nematic and the smectic $A$ phases. Also, there seems to be some indication of pretransitional behavior in the vicinity of the smectic $A$-tosmectic $B$ transition. The Raman depolarization ratios of the same bands as in the last section have been measured as functions of temperature and thickness. Due to intensity considerations, $R_{3}$ in the smectic phases was necessarily obtained using relatively thick samples. We plot in Fig. 20 typical ratios for the $1597 \mathrm{~cm}^{-1}$ band; the data for the other bands are listed in Table IX. The thickness dependence in the nematic phase is quite obvious, though none is observed in the smectic phases. Considering the entire temperature range, the characteristic change of the ratios $R_{1}$ and $R_{2}$ is remarkable. $R_{3}$ is much less sensitive to changes in temperature.

\section{B. Discussion}

Following the analysis described in the last section, we consider the $1597 \mathrm{~cm}^{-1}$ band. In the previous section we took $\beta_{0}$ to be roughly $5^{\circ}$ for both MBBA and $\mathrm{BBCA}$ molecules. With the aid of a space-filled molecular model for 40.8 we estimate the angle $\beta_{0}$ to be rough-

TABLE VIII. Calculated values of $A_{0}^{(2)}, A_{0}^{(4)}, a, b$, the trace, and the anisotropy for the three bands of neat MBBA. Uncertainties include all the statistical uncertainties listed in Table IV and $\beta_{0}=1^{\circ} \pm 1^{\circ}$ for the 1576 and $1625 \mathrm{~cm}^{-1}$ bands, $5^{\circ} \pm 2^{\circ}$ for the $1597 \mathrm{~cm}^{-1}$ band.

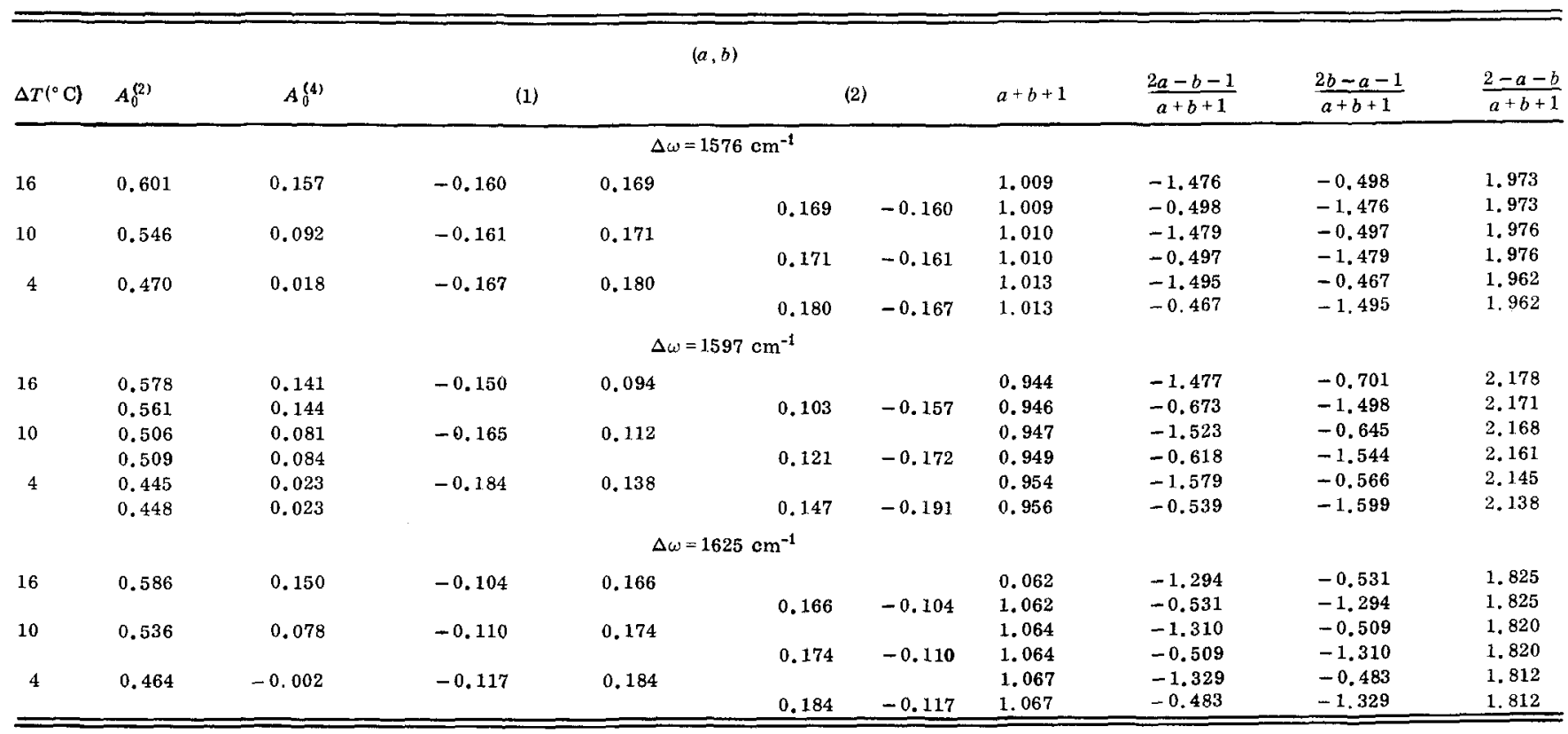




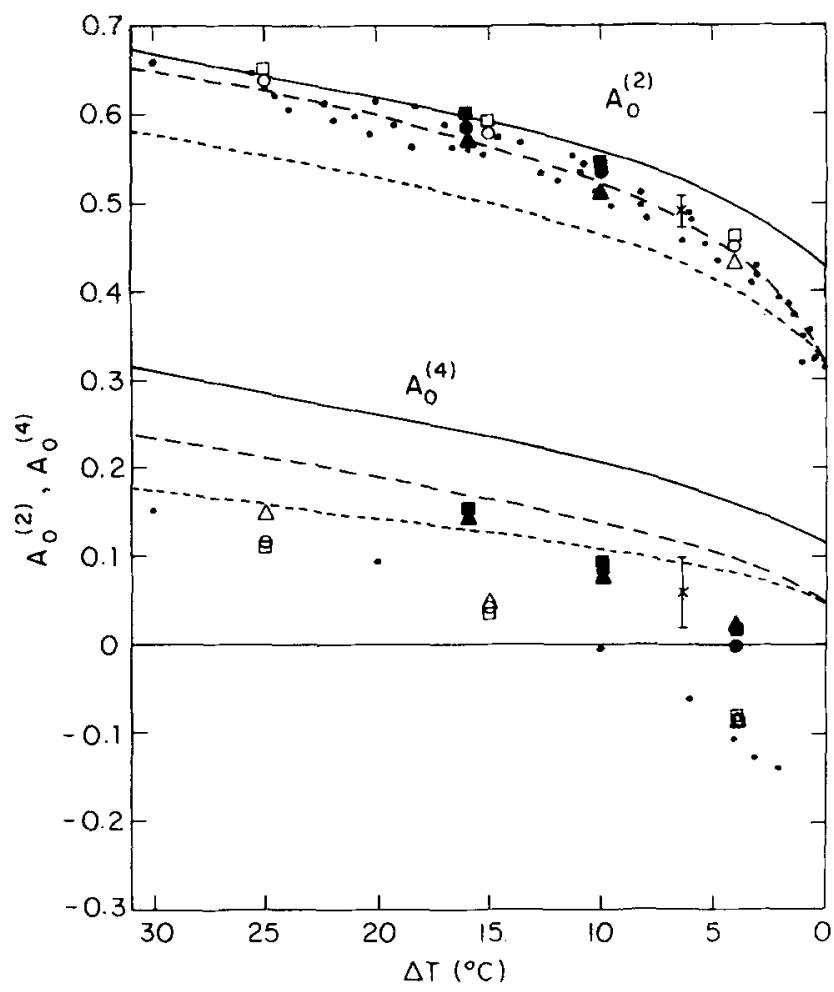

FIG. 18. Order parameters $A_{0}^{(2)}$ and $A_{0}^{(4)}$ deduced from the measurements on the 1576 (squares), 1597 (triangles), and the 1625 (circles) $\mathrm{cm}^{-1}$ bands. The open data points are for the $20 \%$ mixture, and the closed data points for the neat MIBBA. The cross is for the $10 \%$ mixture. Dots are data from Fig. 13. Solid lines: MS theory; dashed lines: HJL theory $(---: m$ $=10 ;---: n=4 ; \lambda=-0.55)$.

ly $10^{\circ}$, assuming the alkoxy and alkyl tails of the 40.8 molecule are pointing along the same direction with respect to the central core as they do in the MBBA molecules. The same calculation yields $A_{0}^{(2)}, A_{0}^{(4)}, a$, and $b$, which are listed in Table X for the case of $\alpha_{0}=0$. Since the angle $\beta_{0}$ is larger than before, the effect of $\alpha_{0}$ may be expected to be nontrivial. However, for different values of $\alpha_{0}$, the calculated values $A_{0}^{(2)}$ and $A_{0}^{(4)}$

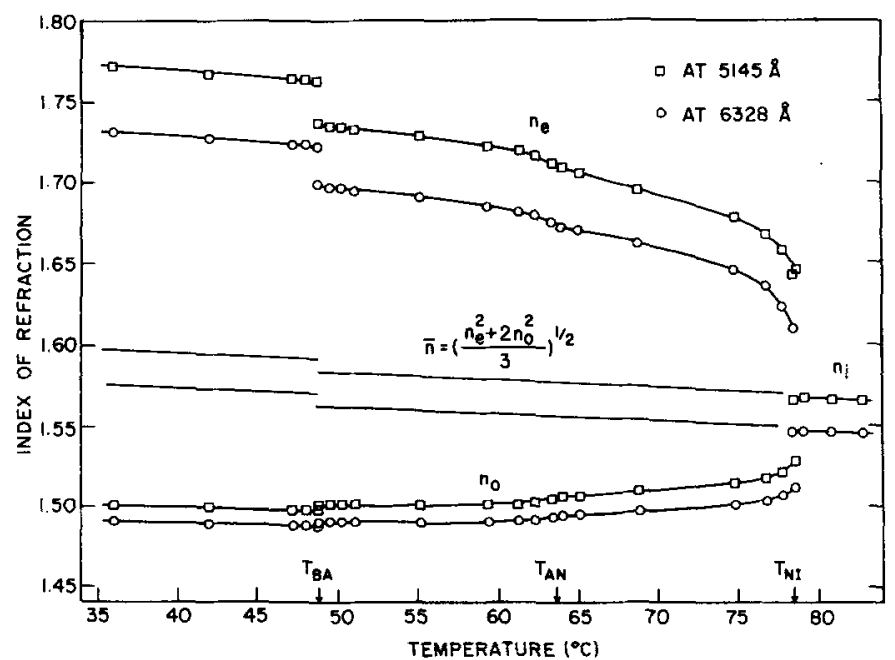

FIG. 19. Indices of refraction of compound 40.8. Data at the lowest temperature are in supercooled smectic $B$ phase.

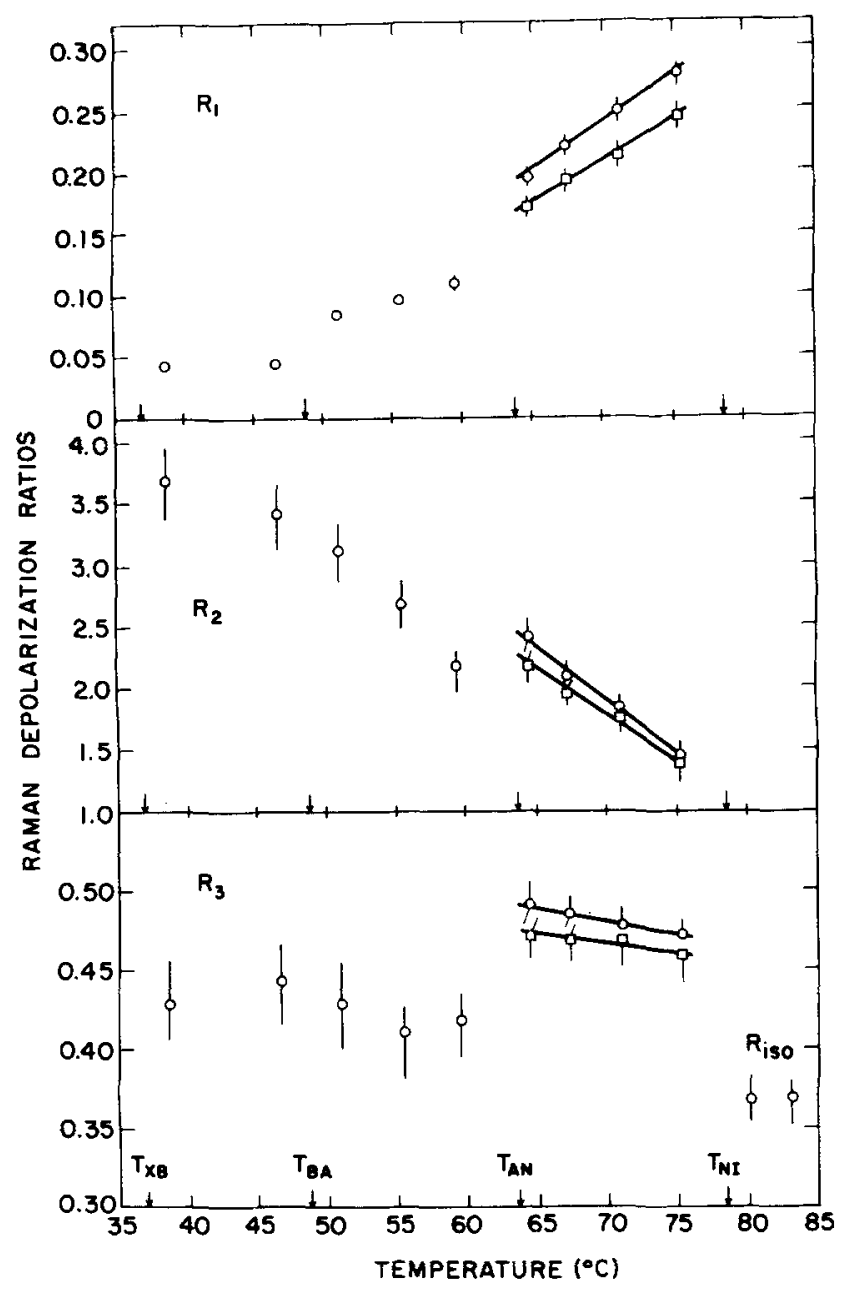

FIG. 20. Depolarization ratios of the $1597 \mathrm{~cm}^{-1}$ band of 40.8 . Squares: $49.6 \mu$; circles: $74.5 \mu$ samples. In smectic $A$ and $B$ phases data include those from $120 \mu$ thick samples.

are not appreciably different from those obtained by setting $\alpha_{0}=0$. The calculated values $a$ and $b$ at fixed $\alpha_{0}$ seem to differ slightly for the nematic and smectic regions; the deviations are much less pronounced when they are expressed in terms of the more physically significant quantities defined by Eqs. (45). The absolute values for $a$ and $b$ are small compared to unity and, although the relative variations appear large, the absolute values of the uncertainties are tolerable when compared with the experimental errors, which include all the statistical uncertainties from the data, $R_{1}, R_{2}, R_{3}$, and $R_{180}$, as well as the estimated value $\beta_{0}$ and the choice of $\alpha_{0}=0$. In addition, if we compare the calculated $A_{0}^{(2)}$ with the relative values obtained from the optical dielectric anisotropy measurements by using the empirical local field correction, the agreement is quite good. Figure 21 is a plot of the Raman data $A_{0}^{(2)}$ and $A_{0}^{(4)}$ obtained above and the relative values of $A_{0}^{(2)}$ obtained by fixing the proportionality factor to give agreement at $75.3^{\circ} \mathrm{C}$. We also show in Fig. 22 a plot similar to that in Fig. 14 , that is, $A_{0}^{(4)}$ vs $A_{0}^{(2)}$. Note that at the lowest temperature the deduced values of $A_{0}^{(2)}$ and $A_{0}^{(4)}$ satisfy the Schwartz inequality and predict value of $\left\langle\sigma^{2}\right\rangle>0$. For nearly complete order one might expect a molecular distribution function 
TABLE IX. Raman depolarization ratios of vibrational bands at 1576 and $1625 \mathrm{~cm}^{-1}$ for 40.8 . Ratios other than $R_{\mathrm{fso}}$ are extrapolated values at zero thickness.

\begin{tabular}{lllll}
\hline $\begin{array}{l}\Delta \omega \\
\left(\mathrm{cm}^{-1}\right)\end{array}$ & $\begin{array}{l}\Delta T \\
\left({ }^{\circ} \mathrm{C}\right)\end{array}$ & \multicolumn{1}{c}{$R_{1}$} & \multicolumn{1}{c}{$R_{2}$} & \multicolumn{1}{c}{$R_{3}$} \\
\hline 1576 & 75.3 & $0.169 \pm 0.016$ & $1.260 \pm 0.060$ & $0.444 \pm 0.020$ \\
& 71.0 & 0.151 & 1.560 & 0.459 \\
& 67.3 & 0.135 & 1.820 & 0.470 \\
& 64.6 & $0.125 \pm 0.016$ & $2.000 \pm 0.200$ & $0.477 \pm 0.031$ \\
& 59.5 & 0.097 & 2.280 & 0.409 \\
& 55.5 & $0.086 \pm 0.006$ & $2.660 \pm 0.160$ & $0.395 \pm 0.026$ \\
& 51.0 & 0.076 & 2.950 & 0.400 \\
& 46.6 & $0.039 \pm 0.001$ & $2.920 \pm 0.130$ & $0.414 \pm 0.028$ \\
& 38.6 & 0.037 & 2.690 & 0.440 \\
& $R_{1 s 0}=$ & $0.337 \pm 0.015$ & & \\
1625 & 75.3 & $0.184 \pm 0.016$ & $1.320 \pm 0.070$ & $0.355 \pm 0.025$ \\
& 71.0 & 0.162 & 1.420 & 0.355 \\
& 67.3 & 0.142 & 1.510 & 0.353 \\
& 64.6 & $0.128 \pm 0.005$ & $1.560 \pm 0.140$ & $0.350 \pm 0.30$ \\
& 59.5 & 0.095 & 1.690 & 0.312 \\
& 55.5 & $0.084 \pm 0.003$ & $1.810 \pm 0.100$ & $0.288 \pm 0.020$ \\
& 51.0 & 0.079 & 1.850 & 0.293 \\
& 46.6 & $0.048 \pm 0.004$ & $1.840 \pm 0.160$ & $0.301 \pm 0.016$ \\
& 38.6 & 0.045 & 2.100 & 0.245 \\
& $R_{\text {1so }}=$ & $0.309 \pm 0.016$ & & \\
\hline
\end{tabular}

$$
f(\beta) \doteq\left\{\begin{array}{l}
A \exp \left(-\beta^{2} / \Delta^{2}\right) \text { for } 0 \leq \beta \leq \pi / 2 \\
A \exp -(\beta-\pi)^{2} / \Delta^{2} \text { for } \pi / 2<\beta \leq \pi
\end{array},\right.
$$

from which the probability of molecules with orientation $\beta$ is given by, for example for $0 \leq \beta \leq \pi / 2$,

$$
f(\beta) \sin \beta d \beta \doteq A \exp \left(-\beta^{2} / \Delta^{2}\right) \beta d \beta .
$$

The normalization of Eq. (46) requires $A=2 / \Delta^{2}$. It follows that and

$$
\left\langle\beta^{2}\right\rangle=\Delta^{2} .\left\langle\beta^{4}\right\rangle=2 \Delta^{4} \text {, etc., }
$$

$$
\left\langle\sigma^{2}\right\rangle \doteq \Delta^{4} /\left(1-\Delta^{2}\right)^{2}, \quad A_{0}^{(2)} \doteq 1-3 \Delta^{2} / 2 .
$$

For $A_{0}^{(2)}=0.92 \pm 0.02$ the predicted value from Eq. (48) for $\left\langle\sigma^{2}\right\rangle$ is $0.005 \pm 0.002$, which is in reasonably good agreement with the value deduced from Fig. 22.

We thus see that the Raman scattering technique ap-

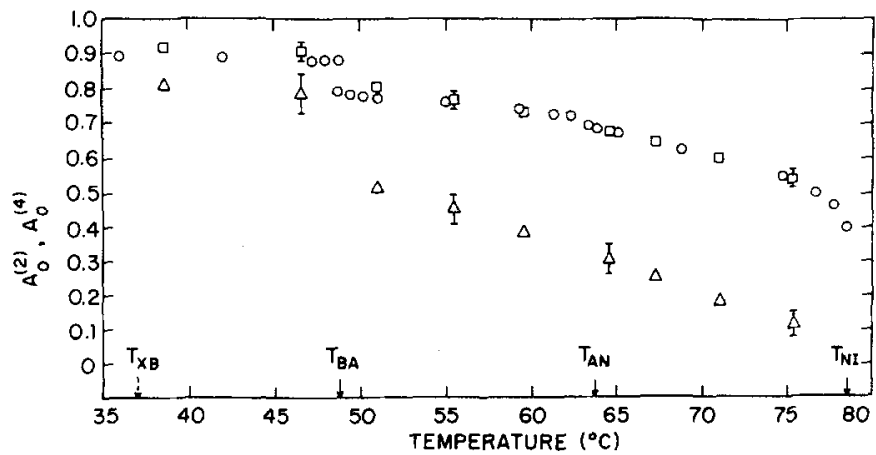

FIG. 21. Order parameters $A_{0}^{(2)}$ (squares) and $A_{0}^{(4)}$ (triangles) calculated from Raman measurements of the $1597 \mathrm{~cm}^{-1}$ band. Circles are relative values obtained from the optical dielectric anisotropy measurements.

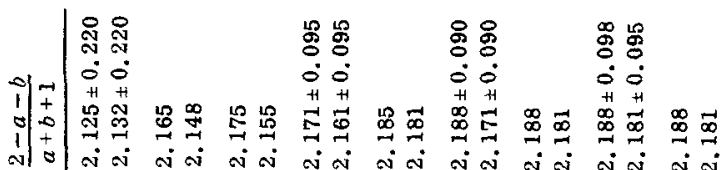

a

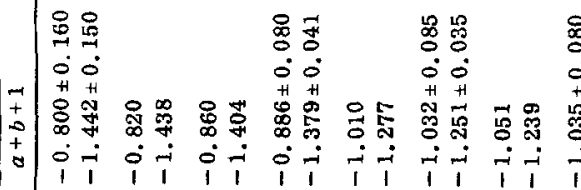

$\stackrel{0}{2}$

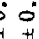

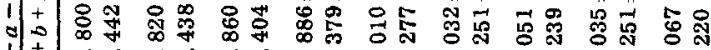

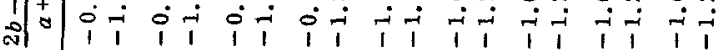

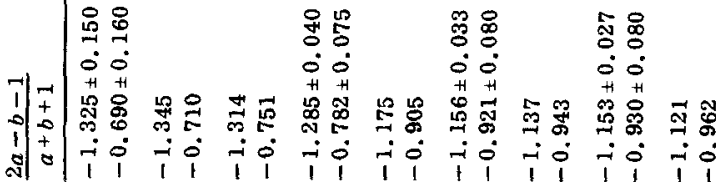

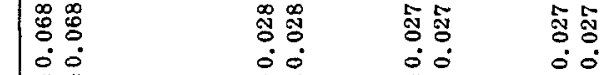

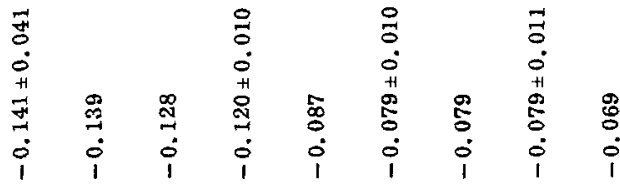

ลิ

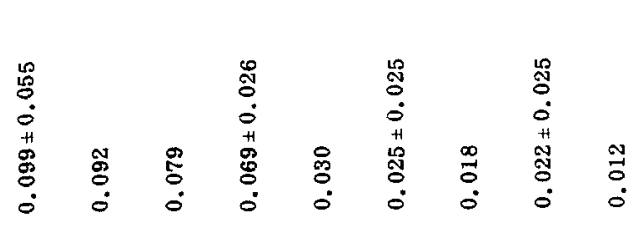

$\Xi$

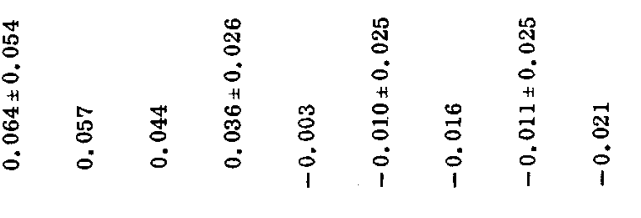




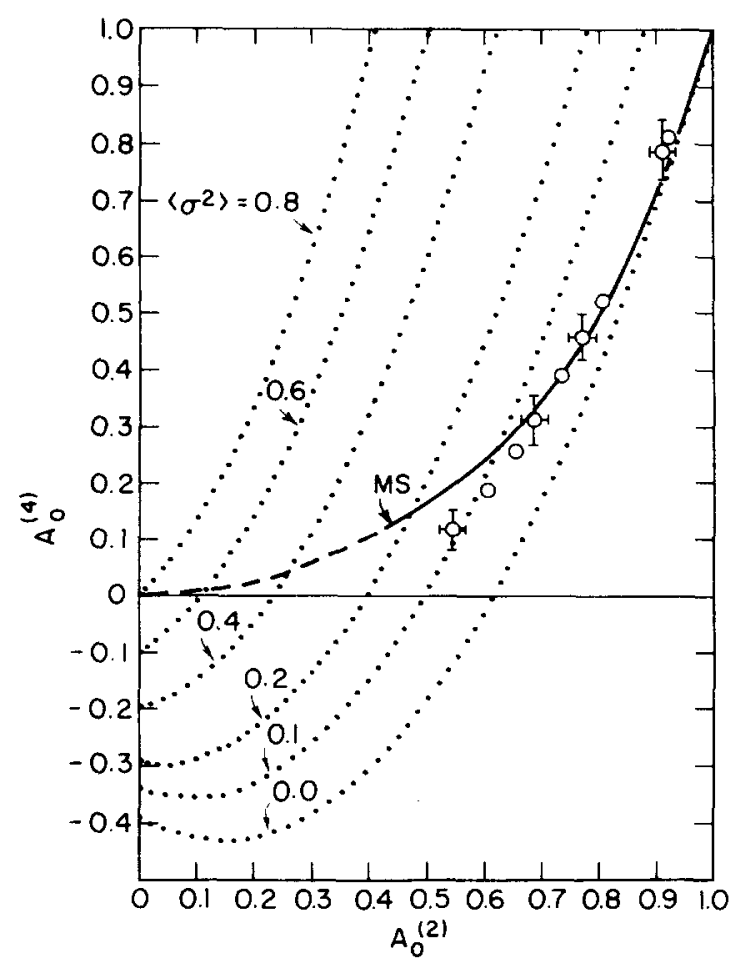

FIG. 22. $A_{0}^{(4)}$ vs $A_{0}^{(2)}$ for compound 40.8. The solid line is from MS theory. The distribution function $\mathrm{Eq}$. (46) gives a variation of $A_{0}^{(4)}$ vs $A_{0}^{(2)}$, which is essentially identical to that from the MS theory for $A_{0}^{(2)} \geq 0.7$. The circles are data points taken from Fig. 25.

pears to be applicable also in the smectic phases. For example, the $A_{0}^{(2)}$ results are in excellent agreement with the optical dielectric anisotropy measurements. Nevertheless, further experimental studies, such as the NMR measurements on partially deuterated molecules, as well as the other independent measurements using different techniques, are required to provide more data. We also learned recently that the EPR line shape of spin probes dissolved in liquid crvstals ${ }^{56}$ has been applied to measure both $A_{0}^{(2)}$ and $A_{0}^{(4)}$ in the nematic phase. This may well give our technique another test.

\section{SUMMARY}

The study of the intensities of the different polarization components of the vibrational Raman spectra of liquid crystals has been unambiguously exploited for the first time. By investigating the well-recognized cyano stretching vibration, the Raman scattering technique has provided new quantitative measurements on the orientational statistics of individual molecules in a nematic liquid crystal. ${ }^{3}$ Subject to the same shortcomings as all the other techniques, this technique has been demonstrated to obtain the same information on the microscopic order parameter $A_{0}^{(2)}$. In addition, new information at the next higher order in the orientational statistics, namely $A_{0}^{(4)}$, has been obtained for the first time. This new quantitative information marks significant discrepancies upon the predictions of existing microscopic theories of nematic ordering.

This technique is further generalized by similar studies using vibrational bands of the neat liquid crystal so that the limited applicability of studying only mixtures is eliminated. This makes the Raman scattering technique readily applicable to essentially all liquid crystals. New data resulting from this general study indicate further the inadequacy of the existing theories.

In smectic $A$ and $B$ phases this technique seems to work reasonably well, although more extensive studies are certainly necessary. In fact, the basic shortcomings that occur in the nematic phase become much less significant here. Immediate applications of this technique to the study of statistics of general molecular groups, such as the end chains of the thermotropic liquid crystal molecules, as well as those of the lyotropic liquid crystal molecules, may be possible.

\section{APPENDIX I}

Expressions for the Raman polarizability tensor elements in the laboratory frame, $\left(\alpha_{L}^{\prime}\right)_{i j}$, in terms of the molecular tensor elements $\alpha_{i j}^{\prime}$ for a molecule of arbitrary orientation $\alpha, \beta, \gamma$ follow. Note that the rotation which carries the molecular frame of reference into the laboratory frame is $R(-\gamma,-\beta,-\alpha)$ (see Fig. 1). The given expressions thus result from the following series of clockwise rotations: $\gamma$ about the 3 -axis, $\beta$ about the new 2-axis, and $\alpha$ about the new 3-axis.

$$
\begin{aligned}
\left(\alpha_{L}^{\prime}\right)_{x x}= & \frac{1}{3}\left(\alpha_{11}^{\prime}+\alpha_{22}^{\prime}+\alpha_{33}^{\prime}\right)+\frac{1}{8}\left(2 \alpha_{33}^{\prime}-\alpha_{11}^{\prime}-\alpha_{22}^{\prime}\right)\left(3 \sin ^{2} \beta \cos ^{2} \alpha-1\right) \\
& +\frac{1}{2}\left(\alpha_{11}^{\prime}-\alpha_{22}^{\prime}\right)\left[\cos 2 \gamma\left(\cos ^{2} \beta \cos ^{2} \alpha-\sin ^{2} \alpha\right)-\sin 2 \gamma \cos \beta \sin 2 \alpha\right]-\alpha_{12}^{\prime}\left[\sin 2 \gamma\left(\cos ^{2} \beta \cos ^{2} \alpha-\sin ^{2} \alpha\right)\right. \\
& +\cos 2 \gamma \cos \beta \sin 2 \alpha]+\alpha_{13}^{\prime} \sin \beta\left(2 \cos \gamma \cos \beta \cos ^{2} \alpha-\sin \gamma \sin 2 \alpha\right)-\alpha_{23}^{\prime} \sin \beta(2 \sin \gamma \cos \beta \cos \alpha+\cos \gamma \sin 2 \alpha), \\
\left(\alpha_{L}^{\prime}\right)_{y y}= & \frac{1}{3}\left(\alpha_{11}^{\prime}+\alpha_{22}^{\prime}+\alpha_{33}^{\prime}\right)+\frac{1}{6}\left(2 \alpha_{33}^{\prime}-\alpha_{11}^{\prime}-\alpha_{22}^{\prime}\right)\left(3 \sin ^{2} \beta \sin ^{2} \alpha-1\right) \\
& +\frac{1}{2}\left(\alpha_{11}^{\prime}-\alpha_{22}^{\prime}\right)\left[\cos 2 \gamma\left(\cos ^{2} \beta \sin ^{2} \alpha-\cos ^{2} \alpha\right)+\sin 2 \gamma \cos \beta \sin 2 \alpha\right]-\alpha_{12}^{\prime}\left[\sin 2 \gamma\left(\cos ^{2} \beta \sin ^{2} \alpha-\cos ^{2} \alpha\right)\right. \\
& -\cos 2 \gamma \cos \beta \sin 2 \alpha]+\alpha_{13}^{\prime} \sin \beta\left(2 \cos \gamma \cos \beta \sin ^{2} \alpha+\sin \gamma \sin 2 \alpha\right)-\alpha_{23}^{\prime} \sin \beta\left(2 \sin \gamma \cos \beta \sin ^{2} \alpha-\cos \gamma \sin 2 \alpha\right), \\
\left(\alpha_{L}^{\prime}\right)_{s z}= & \frac{1}{3}\left(\alpha_{11}^{\prime}+\alpha_{22}^{\prime}+\alpha_{33}^{\prime}\right)+\frac{1}{8}\left(2 \alpha_{33}^{\prime}-\alpha_{11}^{\prime}-\alpha_{22}^{\prime}\right)\left(3 \cos ^{2} \beta-1\right) \\
& +\frac{1}{2} \sin ^{2} \beta\left[\left(\alpha_{11}^{\prime}-\alpha_{22}^{\prime}\right) \cos 2 \gamma-2 \alpha_{12}^{\prime} \sin 2 \gamma\right]-2 \sin \beta \cos \beta\left(\alpha_{13}^{\prime} \cos \gamma-\alpha_{23}^{\prime} \sin \gamma\right), \\
\left(\alpha_{L}^{\prime}\right)_{x y}= & \frac{1}{4}\left(2 \alpha_{33}^{\prime}-\alpha_{11}^{\prime}-\alpha_{22}^{\prime}\right) \sin ^{2} \beta \sin 2 \alpha+\frac{1}{4}\left(\alpha_{11}^{\prime}-\alpha_{22}^{\prime}\right)\left[\cos 2 \gamma\left(1+\cos ^{2} \beta\right) \sin 2 \alpha+2 \sin 2 \gamma \cos \beta \cos 2 \alpha\right] \\
& -\frac{1}{2} \alpha_{12}^{\prime}\left[\sin 2 \gamma\left(1+\cos ^{2} \beta\right) \sin 2 \alpha-2 \cos 2 \gamma \cos \beta \cos 2 \alpha\right]
\end{aligned}
$$


$+\alpha_{13}^{\prime} \sin \beta(\cos \gamma \cos \beta \sin 2 \alpha+\sin \gamma \cos 2 \alpha)-\alpha_{23}^{\prime} \sin \beta(\sin \gamma \cos \beta \sin 2 \alpha-\cos \gamma \cos 2 \alpha)$,

$\left(\alpha_{L}^{\prime}\right)_{x z}=\frac{1}{2}\left(2 \alpha_{33}^{\prime}-\alpha_{11}^{\prime}-\alpha_{22}^{\prime}\right) \sin \beta \cos \beta \cos \alpha-\frac{1}{2}\left(\alpha_{11}^{\prime}-\alpha_{22}^{\prime}\right) \sin \beta(\cos 2 \gamma \cos \beta \cos \alpha-\sin 2 \gamma \sin \alpha)$

$+\alpha_{12}^{\prime} \sin \beta(\sin 2 \gamma \cos \beta \cos \alpha+\cos 2 \gamma \sin \alpha)+\alpha_{13}^{\prime}(\cos \gamma \cos 2 \beta \cos \alpha-\sin \gamma \cos \beta \sin \alpha)$

$-\alpha_{23}^{\prime}(\sin \gamma \cos 2 \beta \cos \alpha+\cos \gamma \cos \beta \sin \alpha)$,

$\left(\alpha_{L}^{\prime}\right)_{y \varepsilon}=\frac{1}{2}\left(2 \alpha_{33}^{\prime}-\alpha_{11}^{\prime}-\alpha_{22}^{\prime}\right) \sin \beta \cos \beta \sin \alpha-\frac{1}{2}\left(\alpha_{11}^{\prime}-\alpha_{22}^{\prime}\right) \sin \beta(\cos 2 \gamma \cos \beta \sin \alpha+\sin 2 \gamma \cos \alpha)$

$+\alpha_{12}^{\prime} \sin \beta(\sin 2 \gamma \cos \beta \sin \alpha-\cos 2 \gamma \cos \alpha)+\alpha_{13}^{\prime}(\cos \gamma \cos 2 \beta \sin \alpha+\sin \gamma \cos \beta \cos \alpha)$

$-\alpha_{23}^{\prime}(\sin \gamma \cos 2 \beta \sin \alpha-\cos \gamma \cos \beta \cos \alpha)$.

\section{APPENDIX $\|$}

\section{A. Order parameters of $L=2$ and 4}

Definitions:

$$
\begin{aligned}
& a_{0 m}^{(L)}=A_{m}^{(L)}+i B_{m}^{(L)}=(-1)^{m} a_{0,-m}^{(L) *}=\left\langle D_{0 m}^{(L) *}(\alpha, \beta, \gamma)\right\rangle, \\
& A_{0}^{(0)}=1, \\
& A_{0}^{(2)}=\frac{1}{2}\left\langle 3 \cos ^{2} \beta-1\right\rangle, \\
& A_{1}^{(2)}=\sqrt{\frac{3}{2}}\langle\sin \beta \cos \beta \cos \gamma\rangle, \\
& B_{1}^{(2)}=\sqrt{\frac{3}{2}}\langle\sin \beta \cos \beta \sin \gamma\rangle, \\
& A_{2}^{(2)}=\frac{1}{2} \sqrt{\frac{3}{2}}\left\langle\sin ^{2} \beta \cos 2 \gamma\right\rangle, \\
& B_{2}^{(2)}=\frac{1}{2} \sqrt{\frac{3}{2}}\left\langle\sin ^{2} \beta \sin 2 \gamma\right\rangle, \\
& A_{0}^{(4)}=\frac{1}{8}\left\langle 35 \cos ^{4} \beta-30 \cos ^{2} \beta+3\right\rangle, \\
& A_{1}^{(4)}=\sqrt{\frac{5}{4}}\left\langle\sin ^{2} \beta \cos \beta\left(7 \cos ^{2} \beta-3\right) \cos \gamma\right\rangle, \\
& B_{1}^{(4)}=\sqrt{\frac{5}{4}}\left\langle\sin ^{2} \beta \cos \beta\left(7 \cos ^{2} \beta-3\right) \sin \gamma\right\rangle, \\
& A_{2}^{(4)}=\frac{1}{4} \sqrt{\frac{5}{2}}\left\langle\sin ^{2} \beta\left(7 \cos ^{2} \beta-1\right) \cos 2 \gamma\right\rangle, \\
& B_{2}^{(4)}=\frac{1}{4} \sqrt{\frac{5}{2}}\left\langle\sin ^{2} \beta\left(7 \cos ^{2} \beta-1\right) \sin 2 \gamma\right\rangle, \\
& A_{3}^{(4)}=\sqrt{\frac{35}{4}}\left\langle\sin ^{3} \beta \cos \beta \cos ^{2} \gamma\right\rangle, \\
& B_{3}^{(4)}=\sqrt{\frac{35}{4}}\left\langle\sin ^{3} \beta \cos \beta \sin 3 \gamma\right\rangle,
\end{aligned}
$$

$$
\begin{aligned}
& A_{4}^{(4)}=\frac{1}{8} \sqrt{\frac{35}{2}}\left\langle\sin ^{4} \beta \cos 4 \gamma\right\rangle, \\
& B_{4}^{(4)}=\frac{1}{8} \sqrt{\frac{35}{2}}\left\langle\sin ^{4} \beta \sin 4 \gamma\right\rangle .
\end{aligned}
$$

\section{B. $\left\langle\left(\alpha_{L}^{\prime}\right\rangle_{\alpha \beta}^{2}\right\rangle$ in terms of the order parameters}

We require the average over all orientations of the square of the tensor components given in Appendix I. For a uniaxial system there are only four independent quantities $\left\langle\left(\alpha_{L}^{\prime}\right)_{x x}^{2}\right\rangle,\left\langle\left(\alpha_{L}^{\prime}\right)_{x y}^{2}\right\rangle,\left\langle\left(\alpha_{L}^{\prime}\right)_{x z}^{2}\right.$, and $\left\langle\left(\alpha_{L}^{\prime}\right)_{z z}^{2}\right\rangle$. These quantities can be expressed in terms of the order param eters of $L=2$ and 4 as in the following. For convenience, we use $\left\langle\cos ^{2} \beta\right\rangle$ and $\left\langle\cos ^{4} \beta\right\rangle$ instead of $A_{0}^{(2)}$ and $A_{0}^{(4)}$, and define the quantities

$$
\begin{aligned}
& A=\alpha_{11}^{\prime}+\alpha_{22}^{\prime}+\alpha_{33}^{\prime}, \\
& B=\left(1 / A^{2}\right)\left[\frac{1}{4}\left(\alpha_{11}^{\prime}-\alpha_{22}^{\prime}\right)^{2}+\alpha_{12}^{\prime 2}\right], \\
& C=\left(1 / A^{2}\right)\left(\alpha_{13}^{\prime 2}+\alpha_{23}^{\prime 2}\right), \\
& D=(1 / A)\left(2 \alpha_{33}^{\prime}-\alpha_{11}^{\prime}-\alpha_{22}^{\prime}\right), \\
& E=\left(1 / A^{2}\right)\left[\frac{1}{4}\left(\alpha_{11}^{\prime}-\alpha_{22}^{\prime}\right)^{2}-\alpha_{12}^{\prime 2}\right] \\
& F=\left(1 / A^{2}\right)\left(\alpha_{13}^{\prime 2}-\alpha_{23}^{\prime 2}\right), \\
& G=\left(1 / A^{2}\right)\left(\alpha_{11}^{\prime}-\alpha_{22}^{\prime}\right) .
\end{aligned}
$$

$$
\begin{aligned}
& A^{-2}\left\langle\left(\alpha_{L}^{\prime}\right)_{x x}^{2}\right\rangle=\frac{1}{9}+\frac{3}{16} B+\frac{1}{4} C+\frac{1}{18} D+\frac{11}{288} D^{2}+\left(\frac{1}{8} B+\frac{1}{2} C-\frac{1}{8} D-\frac{5}{48} D^{2}\right)\left\langle\cos ^{2} \beta\right\rangle+\left(\frac{3}{16} B-\frac{3}{4} C+\frac{3}{32} D^{2}\right)\left\langle\cos ^{4} \beta\right\rangle \\
& +(3 E / \sqrt{70}) A_{4}^{(4)}+\frac{1}{7} F\left(3 \sqrt{\frac{2}{5}} A_{2}^{(4)}-2 \sqrt{\frac{2}{3}} A_{2}^{(2)}\right)-(2 / 3 A) \sqrt{\frac{2}{3}}\left[\frac{1}{2}\left(\alpha_{11}^{\prime}-\alpha_{22}^{\prime}\right) A_{2}^{(2)}-\alpha_{12}^{\prime} B_{2}^{(2)}-\alpha_{13}^{\prime} A_{1}^{(2)}+\alpha_{23}^{\prime} B_{1}^{(2)}\right] \\
& +(D / 21 A)\left\{\left(\alpha_{11}^{\prime}-\alpha_{22}^{\prime}\right)\left(2 \sqrt{\frac{2}{3}} A_{2}^{(2)}+\frac{9}{4} \sqrt{\frac{2}{5}} A_{2}^{(4)}\right)-\alpha_{12}^{\prime}\left(4 \sqrt{\frac{2}{3}} B_{2}^{(2)}+\frac{9}{2} \sqrt{\frac{2}{5}} B_{2}^{(4)}\right)+\alpha_{13}^{\prime}\left[2 \sqrt{\frac{2}{3}} A_{1}^{(2)}-(9 / \sqrt{5}) A_{1}^{(4)}\right]\right. \\
& \left.-\alpha_{23}^{\prime}\left[2 \sqrt{\frac{2}{3}} B_{1}^{(2)}-(9 / \sqrt{5}) B_{1}^{(4)}\right]\right\}+\frac{1}{2} G\left\{-\sqrt{\frac{2}{35}} \alpha_{12}^{\prime} B_{4}^{(4)}+\alpha_{13}^{\prime}\left(\frac{4}{7} \sqrt{\frac{2}{3}} A_{1}^{(2)}+(3 / 7 \sqrt{5}) A_{1}^{(4)}-(3 / \sqrt{35}) A_{3}^{(4)}\right]\right. \\
& \left.+\alpha_{23}^{\prime}\left[\frac{4}{7} \sqrt{\frac{2}{3}} B_{1}^{(2)}+(3 / 7 \sqrt{5}) B_{1}^{(4)}+(3 / \sqrt{35}) B_{3}^{(4)}\right]\right\}+\frac{\alpha_{12}^{\prime}}{A^{2}}\left[\alpha_{13}^{\prime}\left(-\frac{4}{7} \sqrt{\frac{2}{3}} B_{1}^{(2)}-\frac{3}{7 \sqrt{5}} B_{1}^{(4)}+\frac{3}{\sqrt{35}} B_{3}^{(4)}\right)\right. \\
& \left.+\alpha_{23}^{\prime}\left(\frac{4}{7} \sqrt{\frac{2}{3}} B_{1}^{(2)}+\frac{3}{7 \sqrt{5}} A_{1}^{(4)}+\frac{3}{\sqrt{35}} A_{3}^{(4)}\right)\right]+\frac{2 \alpha_{13}^{\prime} \alpha_{23}^{\prime}}{7 A^{2}}\left(2 \sqrt{\frac{2}{3}} B_{2}^{(2)}-3 \sqrt{\frac{2}{5}} B_{2}^{(4)}\right), \\
& A^{-2}\left\langle\left(\alpha_{L}^{\prime}\right)_{x y}^{2}\right\rangle=\frac{1}{16} B+\frac{1}{4} C+\frac{1}{32} D^{2}+\left(\frac{3}{8} B-\frac{1}{16} D^{2}\right)\left\langle\cos ^{2} \beta\right\rangle+\left(\frac{1}{18} B-\frac{1}{4} C+\frac{1}{32} D^{2}\right)\left\langle\cos ^{4} \beta\right\rangle \\
& +(E / \sqrt{70}) A_{4}^{(4)}+\frac{1}{7} F\left(\sqrt{\frac{2}{5}} A_{2}^{(4)}-\sqrt{6} A_{2}^{(2)}\right)+(D / 7 A)\left\{\frac{1}{4}\left(\alpha_{11}^{\prime}-\alpha_{22}^{\prime}\right)\left(4 \sqrt{\frac{2}{3}} A_{2}^{(2)}+\sqrt{\frac{2}{5}} A_{2}^{(4)}\right)\right. \\
& \left.-\frac{1}{2} \alpha_{12}^{\prime}\left(4 \sqrt{\frac{2}{3}} B_{2}^{(2)}+\sqrt{\frac{2}{5}} B_{2}^{(4)}\right)+\alpha_{13}^{\prime}\left[\sqrt{\frac{2}{3}} A_{1}^{(2)}-(1 / \sqrt{5}) A_{1}^{(4)}\right]-\alpha_{23}^{\prime}\left[\sqrt{\frac{2}{3}} B_{1}^{(2)}-(1 / \sqrt{5}) B_{1}^{(4)}\right]\right\} \\
& -\frac{1}{2} G\left\{\sqrt{\frac{g}{35}} \alpha_{12}^{\prime} B_{4}^{(4)}-\alpha_{13}^{\prime}\left[\frac{2}{7} \sqrt{6} A_{1}^{(2)}+(1 / 7 \sqrt{5}) A_{1}^{(4)}-(1 / \sqrt{35}) A_{3}^{(4)}\right]-\alpha_{23}^{\prime}\left[\frac{2}{7} \sqrt{6} B_{1}^{(2)}+(1 / 7 \sqrt{5}) B_{1}^{(4)}+(1 / \sqrt{35}) B_{3}^{(4)}\right]\right\} \\
& -\frac{\alpha_{12}^{\prime}}{A^{2}}\left[\alpha_{13}^{\prime}\left(\frac{2}{7} \sqrt{6} B_{1}^{(2)}+\frac{1}{7 \sqrt{5}} B_{1}^{(4)}-\frac{1}{\sqrt{35}} B_{3}^{(4)}\right)-\alpha_{23}^{\prime}\left(\frac{2}{7} \sqrt{6} A_{1}^{(2)}+\frac{1}{7 \sqrt{5}} A_{1}^{(4)}+\frac{1}{\sqrt{35}} A_{3}^{(4)}\right)\right]+\frac{2 \alpha_{13}^{\prime} \alpha_{23}^{\prime}}{7 A^{2}}\left(\sqrt{6} B_{2}^{(2)}-\sqrt{\frac{2}{5}} B_{2}^{(4)}\right),
\end{aligned}
$$

$A^{-2}\left\langle\left(\alpha_{L}^{\prime}\right)_{x z}^{2}\right\rangle=\frac{1}{4} B+\frac{1}{4} C-\left(\frac{3}{4} C-\frac{1}{8} D^{2}\right)\left\langle\cos ^{2} \beta\right\rangle-\left(\frac{1}{4} B-C+\frac{1}{8} D^{2}\right)\left\langle\cos ^{4} \beta\right\rangle-(4 E / \sqrt{70}) A_{4}^{(4)}+\frac{1}{14} F\left(\sqrt{6} A_{2}^{(2)}-8 \sqrt{\frac{2}{5}} A_{2}^{(4)}\right)$ 


$$
\begin{aligned}
& -\frac{D}{14 A}\left(\alpha_{11}^{\prime}-\alpha_{22}^{\prime}\right)\left(\sqrt{\frac{2}{3}} A_{2}^{(2)}+2 \sqrt{\frac{2}{5}} A_{2}^{(4)}\right)-2 \alpha_{12}^{\prime}\left(\sqrt{\frac{2}{3}} B_{2}^{(2)}+2 \sqrt{\frac{2}{5}} B_{2}^{(4)}\right)+\alpha_{13}^{\prime}\left(\sqrt{\frac{2}{3}} A_{1}^{(2)}-\frac{8}{\sqrt{5}} A_{1}^{(4)}\right)-\alpha_{23}^{\prime}\left(\sqrt{\frac{2}{3}} B_{1}^{(2)}-\frac{8}{\sqrt{5}} B_{1}^{(4)}\right) \\
& +G\left\{2 \sqrt{\frac{2}{35}} \alpha_{12}^{\prime} B_{4}^{(4)}-\frac{1}{2} \alpha_{13}^{\prime}\left[\frac{1}{7} \sqrt{6} A_{1}^{(2)}+(4 / 7 \sqrt{5}) A_{1}^{(4)}-(4 / \sqrt{35}) A_{3}^{(4)}\right]-\frac{1}{2} \alpha_{23}^{\prime}\left[\frac{1}{7} \sqrt{6} B_{1}^{(2)}+(4 / 7 \sqrt{5}) B_{1}^{(4)}+(4 / \sqrt{35}) B_{3}^{(4)}\right]\right\} \\
& +\frac{\alpha_{12}^{\prime}}{A^{2}}\left[\alpha_{13}^{\prime}\left(\frac{\sqrt{6}}{7} B_{1}^{(2)}+\frac{4}{7 \sqrt{5}} B_{1}^{(4)}-\frac{4}{\sqrt{35}} B_{3}^{(4)}\right)-\alpha_{23}^{\prime}\left(\frac{\sqrt{6}}{7} A_{1}^{(2)}+\frac{4}{7 \sqrt{5}} A_{1}^{(4)}+\frac{4}{\sqrt{35}} A_{3}^{(4)}\right)\right]+\frac{\alpha_{13}^{\prime} \alpha_{23}^{\prime}\left(\sqrt{6} B_{2}^{(2)}-8 \sqrt{\frac{2}{5}} B_{2}^{(4)}\right),}{7 A^{2}}
\end{aligned}
$$$$
A^{-2}\left\langle\left(\alpha_{L}^{\prime}\right)_{z z}^{2}\right\rangle=\frac{1}{9}+\frac{1}{2} B-\frac{1}{9} D+\frac{1}{36} D^{2}-\left(B-2 C-\frac{1}{3} D+\frac{1}{6} D^{2}\right)\left\langle\cos ^{2} \beta\right\rangle+\left(\frac{1}{2} B-2 C+\frac{1}{4} D^{2}\right)\left\langle\cos ^{4} \beta\right\rangle+4 E \sqrt{\frac{2}{35}} A_{4}^{(4)}
$$$$
+\frac{4}{7} F\left(\sqrt{\frac{2}{3}} A_{2}^{(2)}+2 \sqrt{\frac{2}{5}} A_{2}^{(4)}\right)+(2 / 3 A) \sqrt{\frac{2}{3}}\left[\left(\alpha_{11}^{\prime}-\alpha_{22}^{\prime}\right) A_{2}^{(2)}-2 \alpha_{12}^{\prime} B_{2}^{(2)}-2 \alpha_{13}^{\prime} A_{1}^{(2)}-2 \alpha_{23}^{\prime} B_{1}^{(2)}\right]+\frac{2 D}{21 A}
$$$$
\times\left[\left(\alpha_{11}^{\prime}-\alpha_{22}^{\prime}\right)\left(-2 \sqrt{\frac{2}{3}} A_{2}^{(2)}+3 \sqrt{\frac{2}{5}} A_{2}^{(4)}\right)-2 \alpha_{12}^{\prime}\left(-2 \sqrt{\frac{2}{3}} B_{2}^{(2)}+3 \sqrt{\frac{2}{5}} B_{2}^{(4)}\right)-2 \alpha_{13}^{\prime}\left(\sqrt{\frac{2}{3}} A_{1}^{(2)}+\frac{6}{\sqrt{5}} A_{1}^{(4)}\right)+2 \alpha_{23}^{\prime}\left(\sqrt{\frac{2}{3}} B_{1}^{(2)}+\frac{6}{\sqrt{5}} B_{1}^{(4)}\right)\right]
$$$$
-4 G\left\{\sqrt{\frac{2}{35}} \alpha_{12}^{\prime} B_{4}^{(4)}+\alpha_{13}^{\prime}\left[\frac{1}{7} \sqrt{\frac{2}{3}} A_{1}^{(2)}-(1 / 7 \sqrt{5}) A_{1}^{(4)}+(1 / \sqrt{35}) A_{3}^{(4)}\right]-\alpha_{23}^{\prime}\left[\frac{1}{7} \sqrt{\frac{2}{3}} B_{1}^{(2)}-(1 / 7 \sqrt{5}) B_{1}^{(4)}+(1 / \sqrt{35}) B_{3}^{(4)}\right]\right\}
$$$$
+\frac{8 \alpha_{12}^{\prime}}{A^{2}}\left[\alpha_{13}^{\prime}\left(\frac{1}{7} \sqrt{\frac{2}{3}} B_{1}^{(2)}-\frac{1}{7 \sqrt{5}} B_{1}^{(4)}\right)+\sqrt{\frac{1}{35}} B_{3}^{(4)}-\alpha_{23}^{\prime}\left(\frac{1}{7} \sqrt{\frac{2}{3}} A_{1}^{(2)}-\frac{1}{7 \sqrt{5}} A_{1}^{(4)}-\frac{1}{\sqrt{35}} A_{3}^{(4)}\right)\right]-\frac{8 \alpha_{13}^{\prime} \alpha_{23}^{\prime}}{7 A^{2}}\left(\sqrt{\frac{2}{3}} B_{2}^{(2)}+2 \sqrt{\frac{2}{5}} B_{2}^{(4)}\right) \text {. }
$$

\section{APPENDIX III. EVALUATION OF $A_{0}^{(2)}, A_{0}^{(4)}, a$, and $b$}

From Eqs. (27), (37), and (38) and the experimental depolarization ratios we have the following system of equations which are linear in the order parameters $A_{0}^{(2)}$ and $A_{0}^{(4)}$ :

$$
\begin{aligned}
& a_{11}\left\langle\cos ^{2} \beta\right\rangle+a_{12}\left\langle\cos ^{4} \beta\right\rangle=c_{1}, \\
& a_{21}\left\langle\cos ^{2} \beta\right\rangle+a_{22}\left\langle\cos ^{4} \beta\right\rangle=c_{2}, \\
& a_{31}\left\langle\cos ^{2} \beta\right\rangle+a_{32}\left\langle\cos ^{4} \beta\right\rangle=c_{3},
\end{aligned}
$$

where

$$
\begin{array}{ll}
a_{11}=x_{8}-r_{1} x_{11}, & a_{12}=x_{a}-r_{1} x_{12}, \\
a_{21}=x_{8}-r_{2} x_{2}, & a_{22}=x_{a}-r_{2} x_{3}, \\
a_{31}=x_{5}-r_{3} x_{2}, & a_{32}=x_{6}-r_{3} x_{3}, \\
c_{1}=r_{1} x_{10}-x_{7}, & c_{2}=r_{2} x_{1}-x_{7}, \\
c_{3}=r_{3} x_{1}-x_{4} &
\end{array}
$$

and $r_{1}, r_{2}$, and $r_{3}$ are the ratios after the correction of surface coupling factor [Eq. (22)], and for $k=1,4,7,10$

$$
x_{k}=x_{k}\left(a, b, \alpha_{0}, \beta_{0}, A_{1}^{(2)}, A_{2}^{(4)}, A_{4}^{(4)}\right)
$$

and for the rest of $k$

$$
x_{k}=x_{k}\left(a, b, \alpha_{0}, \beta_{0}\right) \text {. }
$$

All the $x_{k}$ are directly related to the entities in Eq. (38) and the coefficients in Eq. (27). It is worthwhile to notice that all the $x_{k}$ are second order polynomials in $a$ and $b$, which implies that Eq. (AIII.1) are also second order in $a$ and $b$. We can get a relation between $a$ and $b$ by eliminating $\left\langle\cos ^{2} \beta\right\rangle$ and $\left\langle\cos ^{4} \beta\right\rangle$ from Eq. (AIII.1),

$$
\begin{aligned}
\left(c_{1}-a_{11} c_{3} / a_{31}\right)\left(a_{22}\right. & \left.-a_{21} a_{32} / a_{31}\right) \\
& =\left(c_{2}-a_{21} c_{3} / a_{31}\right)\left(a_{12}-a_{11} a_{32} / a_{31}\right)
\end{aligned}
$$

with $\alpha_{0}, \beta_{0}$, etc., being kept as adjustable parameters. The depolarization ratio in the isotropic phase $R_{180}$ provides another relation between $a$ and $b$, that is, Eq. (28) or

$$
a=\left[1 / 2\left(3 R_{190}-1\right)\right]\left[-(1+b)\left(2 R_{180}+1\right)\right.
$$

$$
\begin{aligned}
& \pm\left\{(1+b)^{2}\left(2 R_{\mathrm{iso}}+1\right)^{2}-4\left(3 R_{\mathrm{iso}}-1\right)\right. \\
& \left.\left.\times\left[\left(1+b^{2}\right)\left(3 R_{\mathrm{iso}}-1\right)+b\left(2 R_{\mathrm{iso}}+1\right)\right]\right\}^{1 / 2}\right] .
\end{aligned}
$$

Equation (AIII. 3) is plotted in Fig. 23 for a number of values of $R_{150}$, where the contours may be an ellipse $\left(R_{\mathrm{iso}}<\frac{1}{8}\right)$, a parabola $\left(R_{\mathrm{iso}}=\frac{1}{8}\right)$, or a hyperbola $\left(R_{\mathrm{iso}}>\frac{1}{8}\right)$. As the functional dependence of Eq. (AIII.2) on $a$ and $b$ is quite complicated, we choose to solve Eqs. (AIII.2) and (AIII. 3) for $a$ and $b$ in the following way. For a given $R_{\text {iso }}$, a value for $a$ is obtained from Eq. (AIII.3) with an arbitrary value of $b$. This pair of $a$ and $b$ are then substituted into both sides of Eq. (AIII. 2) with the parameters $\alpha_{0}, \beta_{0}$, etc., being chosen beforehand. By comparing both sides of Eq. (AIII. 2), for successive values of $b$, we can eventually locate the specific $b$, with corresponding $a$, for which Eq. (AIII.2) is satisfied. As both Eqs. (AIII.2) and (AIII. 3) are second order in $a$ and $b$, we expect at most four sets of solutions $(a, b)$. However, using values of $b$ ranging from -10 to 10 we get for all the cases only two sets of real solutions with

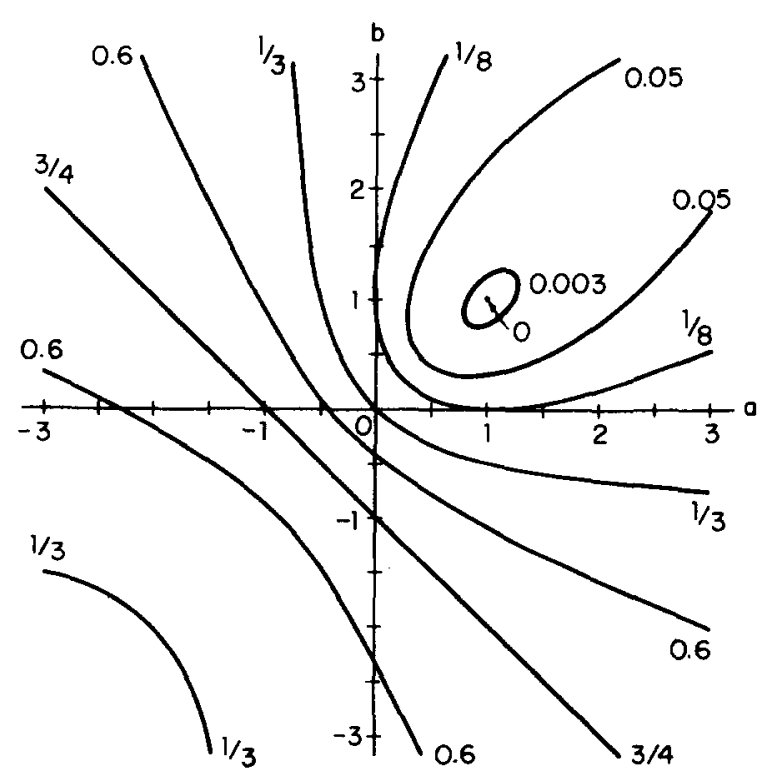

FIG, 23. Contours of constant $\boldsymbol{R}_{180}$ in the $(a, b)$ plane. 
the experimental depolarization ratios. For the special cases, like with $\alpha_{0}=0$ or $a=b$ (uniaxial), the simplification of this algorithm is straightforward. The actual computer program that was written for the Xerox SDS Sigma 7 computer is available in the $\mathrm{Ph}$. D. thesis of one of us (SJ). ${ }^{41}$

*Work supported in part by the Joint Services Electronics Program (U. S. Army, Navy, and Air Force) under Contract No. N00014-75-C-0648 and by the National Science Foundation under Grants No. DMR-72-02088 and DMR72-03020-A05.

tPresent address: Department of Chemistry, State University of New York, Stony Brook, New York 11.794.

${ }^{1}$ W. Maier and A. Saupe, Z. Naturforsch. 14a, 882 (1.959); 15a, 287 (1960).

${ }^{2}$ R. L. Humphries, P. G. James, and G, R. Luckhurst, J。 Chem. Soc. Faraday Trans. II 68, 1031 (1972); G. R. Luckhurst, Symp. Faraday Soc. 5, 46 (1971).

${ }^{3}$ S. Jen, N. A. Clark, P. S. Pershan, and E. B. Priestley, Phys. Rev. Lett. 31, 1552 (1973).

${ }^{4}$ P. J. Flory and Leonard, J. Am. Chem. Soc. 87, 2102 (1965); A. Wulf and A. G. DeRocco, J. Chem. Phys. 55, 12 (1971).

${ }^{5}$ R. Alben, J. McColl, and C. S. Shih, Solid State Commun. 11, 1081. (1972).

${ }^{6}$ (a) B. Deloche and J. Charvolin, presentation at the Vth int. Conf. Liquid Cryst. (Stockholm, Sweden, 1974); (b) S. Meiboom and Z. Luz, Mol. Cryst. Liquid Cryst. 22, 143 (1973). ${ }^{2} \mathrm{P}$. Sheng and P. J. Wojtowicz, to be published.

${ }^{8} \mathrm{M}$. E. Rose, Elementary Theory of Angular Momentum (Wiley: New York, 1957); A. R. Edmonds, Angular Momentum in Quantum Mechanics (Princeton University, Princeton, 1957). Our convention for the Euler angles coincides with that described on $\mathrm{p} .50$ of the book by Rose.

${ }^{9}$ A. Saupe, Z. Naturforsch. 19a, 161 (1964).

${ }^{10}$ L. C. Snyder, J. Chem. Phys. 43, 4041 (1965).

${ }^{11}$ P. G. de Gennes, Phys. Lett. 30A, 454 (1969); Mol. Cryst. Liquid Cryst. 12, 193 (1971).

${ }^{12}$ In our convention the laboratory coordinate system is transformed into the molecule fixed system by a rotation $R(\alpha, \beta, \gamma)$; the inverse transformation is effected by a rotation

$R(-\gamma,-\beta,-\alpha)$. Thus, a second rank tensor $\boldsymbol{\alpha}$ in the molecula frame is transformed to $\alpha_{L}$ in the laboratory frame according to

$$
\begin{aligned}
& \quad \boldsymbol{\alpha}_{L}=\alpha(0,0) \hat{\boldsymbol{\beta}}_{0}^{(0)}+\sum_{m} \hat{\boldsymbol{\beta}}_{m}^{(2)} \sum_{m^{\prime}} \alpha\left(2, m^{\prime}\right) D_{m^{\prime} m}^{(2)}(-\gamma,-\beta,-\alpha) . \\
& \text { But } \\
& \quad D_{m^{\prime} m}^{(2)}(-\gamma,-\beta,-\alpha)=\boldsymbol{D}_{m m^{\prime}}^{(2) *}(\alpha, \beta, \gamma),
\end{aligned}
$$

and

$$
\alpha(0,0) \hat{\boldsymbol{\beta}}_{0}^{(0)} \equiv \frac{1}{3} \mid \operatorname{tr} \alpha,
$$

whence

$$
\boldsymbol{\alpha}_{L}^{\boldsymbol{a}}=\sum_{m} \hat{\boldsymbol{\beta}}_{m}^{(2)} \sum_{m^{\prime}} \alpha\left(2, m^{\prime}\right) D_{m m^{(}}^{(2)^{*}}(\alpha, \beta, \gamma) .
$$

For a uniaxial nematic phase there can be no $\alpha$ dependence (see Fig. 1) so that only the $m=0$ term contributes and we have

$$
\alpha_{L}^{a}=\hat{\beta}_{0}^{(2)} \sum_{m^{\prime}} \alpha\left(2, m^{\prime}\right) D_{0 m^{\prime}}^{(2) *}(\alpha, \beta, \gamma) \text {. }
$$

${ }^{13}$ (a) H. Meuller, Phys. Rev. 47, 947 (1935); 50, 547 (1936); (b) M. Born and K. Huang, Dynamical Theory of Crystal Lattices (Oxford University, London, 1954), Sec. 30 .

${ }^{14}$ See, for example, P. Chatelain, Bull. Soc. Franc. Miner. Cryst. 78, 262 (1955); D. A. Dunmur, Chem. Phys. Lett. 10, 49 (1971); W. Maier and A. Saupe, Z. Naturforsch. 16a, 816 (1961).

${ }^{15} \mathrm{~S}$. Chandrasckhar and N. V. Madhusudana, J. Phys. 30, C424 (1969).

${ }^{16}$ M. F. Vuks, Opt. Spectrosc. 20, 361 (1966).

${ }^{17}$ A. J. Leadbetter, R. M. Richardson, and C. N. Colling, presentation at the Vth Int. Conf. Liquid Cryst. (Stockholm, Sweden, 1974).

${ }^{18}$ (a) H. Zwetkov, Acta Physicochimica, URSS X and XI (1939); (b) A. Saupe and W. Maier, Z. Naturforsch. 16a, 816 (1961); (c) H. Gasparoux, B. Regaya, and J. Prost, C. R. Acad. Sci. 272B, 1168 (1971); H. Gasparoux and J. Prost, J. Phys. 32, 953 (1971); G. Sigaud and H. Gasparoux, J. Chim. Phys. 70,699 (1973).

${ }^{19} \mathrm{H}$. Gasparoux, F. Hardouin, M. F. Achard, and G. Sigaud, presentation at the Vth Int. Conf. Liquid Cryst. (Stockholm, Sweden, 1974).

${ }^{20}$ (a) See for example, Ref. 10. (b) S. Meiboom and Z. Luz, Mol. Cryst. Liquid Cryst. 22, 143 (1973).

${ }^{21}$ See, for example, G. R. Luckhurst, Mol. Cryst. Liquid Cryst. 21, 125 (1973).

${ }^{22}$ H. Lippmann, Ann. Phys. Lpz. 2, 287 (1958); K. H. Weber, ibid. 3, 1 (1959); P. Pincus, J. Phys. 30, C4-8 (1969).

${ }^{23}$ J. W. Doane, R. S. Parker, B. Cvikl, D. L. Johnson, and D. L. Fishel, Phys. Rev. Lett. 28, 1694 (1972).

${ }^{24}$ (a) J. C. Rowell, W. D. Phillips, L. R. Melby, and M. Panar, J. Chem. Phys. 43, 3442 (1965). (b) Y. S. Lee, Y. Y. Hsu, and D. H. Dolphin, presentation at the ACS Symposium on Ordered Fluids and Liquid Crystals (Chicago, 1973). (c) B. Deloche and J. Charvolin, presentation at the Vth Int. Conf. Liquid Cryst. (Stockholm, Sweden, 1974). See also Ref. (20b).

${ }^{25}$ Reference (24a); B. Cabane and W. G. Clarke, Phys. Rev. Lett. 25, 91 (1970).

${ }^{26}$ Reference (15); N. V. Madhusudana, R. Shashidhar, and S. Chandrasekhar, Mol. Cryst. Liquid Cryst. 13, 61 (1971).

${ }^{27}$ Reference (18b); the data here are probably not correct due to the questionable local field correction used.

${ }^{28} \mathrm{G}$. Baur, A. Stieb, and G. Meier, Mol. Cryst. Liquid Cryst. 22,261 (1973).

${ }^{29}$ P. G. de Gennes, C. R. Acad. Sci. 274B, 142 (1972).

${ }^{30}$ I. G. Chistyakov, Sov. Phys. Uspekhi 9, 551 (1967); P. Delord, J. Phys $30, \mathrm{C} 4-14$ (1969).

${ }^{31}$ E. B. Priestley, P. S. Pershan, R. B. Meyer, and D. H. Dolphin, Raman Mem. Vol. Vijnana Parishad Anusandhan Patrika 14, 93 (1971).

${ }^{32}$ (a) G. Placzek, Handbuch der Radiologie, edited by E. Marx, Lpz., Akad. Verlag. VI 2, 209 (1934). (b) Ref. 12b, Chap. III and III.

${ }^{33}$ (a) M. Lax and D. F. Nelson, Phys. Rev. B 4, 3694 (1971). (b) M. Lax and D. F. Nelson, Proceedings of the Third Rochester Conference on Coherent and Quantum Optics, edited by L. Mandel and E. Wolf (Plenum, New York, 1973).

${ }^{34}$ The concentration here and henceforth is given as a percentage by weight. Multiply wt \% by 0.96 to obtain mol \%.

${ }^{35}$ The MBBA and BBCA were prepared by Dr. Z. Muljiani, and the 40.3 by Dr. Y. Y. Hsu, both of Professor D. H. Dolphin's group.

${ }^{36}$ Vari-Light Corporation, Room Temperature Nematic Liquid Crystal MBBA, data sheet 060170 .

${ }^{37}$ L. Verbit, Mol. Cryst. Liquid Cryst. 15, 89 (1971).

${ }^{38}$ G. W. Smith, Z. G. Gardlund, and R. J. Curtis, Mol. Cryst. Liquid Cryst. 19, 327 (1973).

${ }^{39} \mathrm{Dr}$. P. C. Chou of Professor Turnbull's group helped to obtain the DSC data. Our measurements agree with those of $\mathrm{G}_{\text {. }}$ W. Smith and Z. G. Gardlund, J. Chem. Phys. 59, 3214 (1973).

${ }^{40}$ See, for example, M. Born and E. Wolf, Principles of Optics (Oxford University, London, 1970), 4th ed. Chap. XIV.

${ }^{41} \mathrm{~S}$. Jen, Ph. D. thesis, Division of Engineering and Applied Physics, Harvard University (1975).

${ }^{42}$ See, for example, N. M. Amer, Y. R. Shen, and H. Rosen, Phys. Rev. Lett. 24, 718 (1970); W. J. Borer, S. S. Mitra, and C. W. Brown, ibid. 27, 379 (1971); C. H. Wang and A. L. Leu, J. Am. Chem. Soc. 94, 8605 (1972); B. Lavrencic and S. Lugomer, private communication.

${ }^{43}$ The contribution to the thickness dependence from absorption 
has been shown to be orders of magnitude smaller than that due to director fluctuations in MBBA, C. Hu and J. R. Whinnery, private communication.

${ }^{44} \mathrm{Y}$. Beers, Introduction to the Theory of Error (Addison-Wesley, Reading, MA, 1962), 2nd ed.

${ }^{45} \mathrm{P}$. Sheng, Solid State Commun, accepted for publication.

${ }^{46}$ The choice of $\cos 2 \gamma$ maintains the required symmetry relation for which Eq. (4) is valid, that is, $f(\gamma)=f(\pi-\gamma)=f(\pi+\gamma)$.

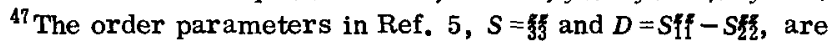
equivalent in our notation to

$$
\begin{aligned}
& S=\frac{1}{2}\left\langle 3 \cos ^{2} \beta-1\right\rangle=A_{0}^{(2)}, \\
& D=\frac{3}{2}\left\langle\sin ^{2} \beta \cos 2 \gamma\right\rangle=2 \sqrt{\frac{3}{2}} A_{2}^{(2)} .
\end{aligned}
$$

A value of $D=0.07$ with $S=0.52$ at $120^{\circ} \mathrm{C}$ for PAA corresponds to $\delta=0.30$, under the assumption of Eq. (39).

${ }^{48}$ The empirical relation in Ref. $5, D=0.15(1-S)$, is a direct consequence of Eq. (39) with $\delta=0.3$. The value of $\delta$ could be quite sensitive to structure, in which case the value would be transferable from material to material. However, in the absence of such knowledge, we have used $\delta=0.3$ together with zeroth order estimates of $\left\langle\sin ^{2} \beta\right\rangle$ and $\left\langle\sin ^{2} \beta\left(7 \cos ^{2} \beta-1\right)\right\rangle$ to calculate corrections to $A_{0}^{(2)}$ and $A_{0}^{(4)}$ for our system.

${ }^{49}$ From Eq. (13) we have

$$
(\bar{\epsilon}-1) /(\bar{\epsilon}+2)=\frac{4}{3} \pi N_{0} \bar{\alpha} \text {. }
$$

However, $\left.V \propto 1 / N_{0} \propto(\epsilon+2) / \epsilon-1\right)$,

whence

$$
V^{*}=\frac{[\bar{\epsilon}(T)+2] /[\bar{\epsilon}(T)-1]}{\left[\bar{\epsilon}\left(T_{N I}\right)+2\right] /\left[\bar{\epsilon}\left(T_{N I}\right)-1\right]}
$$

with $\bar{\epsilon}\left(T_{N I}\right)$ being the value at $T_{N I}$ in the nematic phase.

${ }^{50} \mathrm{P}$. I. Rose, presentation at the IVth International Conference on Liquid Crystals (Kent, Ohio, 1972).

${ }^{51}$ R. L. Humphries, P. G. James, and G. R. Luckhurst, Symp. Faraday Soc. 5, 107 (1971); this reference develops a mean field theory of liquid crystal mixtures based on Maier-Saupe theory. For our mixture this new theory gives essentially the same predictions as does MS theory because of the nearly identical dependence of $V^{*}$ (Fig. 17) for the mixture and neat MBBA. For mixtures with components having different functional dependence of $V^{*}$ on $T^{*}$, the derivation from MS theory can be significant.

${ }^{52}$ This correction can readily be included since the required quantity $|\Delta V| / V\left(T_{N I}\right)$ can also be obtained from the refractive index data.

${ }^{53}$ R. Pynn, Solid State Commun. 14, 29 (1974); J. Chem. Phys. 60,4579 (1974).

${ }^{54}$ T. D. Gierke and W. H. Flygare, J. Chem. Phys. 61, 2231 (1974).

${ }^{55}$ There seems to be no noticeable difference in the Raman shifts of these bands among the Schiff's base compounds we have looked at, within the accuracy of our spectra, which were taken with an instrumental resolution of $4 \mathrm{~cm}^{-1}$. Unlike in the infrared spectra (see, for example, B. J. Bulkin, T. Kennelly, and W. B. Lok, presentation at the ACS Symposium on Ordered Fluids and Liquid Crystals, Chicago, 1973) we do not resolve two bands near $1597 \mathrm{~cm}^{-1}$ for any of the compounds except CBOOA, for which an additional band is observed at $1604 \mathrm{~cm}^{-1}$.

${ }^{56}$ G. R. Luckhurst and R. Poupko, private commmunication (to be published). 